\title{
Combinatorial Independence and Sofic Entropy
}

\author{
David Kerr • Hanfeng Li
}

Received: 31 December 2012 / Accepted: 22 January 2013 / Published online: 22 February 2013 (C) School of Mathematical Sciences, University of Science and Technology of China and Springer-Verlag Berlin Heidelberg 2013

\begin{abstract}
We undertake a local analysis of combinatorial independence as it connects to topological entropy within the framework of actions of sofic groups.
\end{abstract}

Keywords Independence · Sofic entropy · Loeb space · Algebraic action · Fuglede-Kadison determinant $\cdot$ Li-Yorke chaos

\section{Introduction}

Among the various phenomena in dynamics associated with randomness, weak mixing and entropy stand out for the depth of their theory and the breadth of their applications (see for example [11, 13]). In the setting of discrete acting groups, weak mixing makes sense in general while entropy, as classically formulated, requires the group to be amenable. One can view these two concepts in a unified way across both measurable and topological dynamics by means of the combinatorial notion of independence. In close parallel with the $\ell_{1}$ theorems of Rosenthal and Elton-Pajor in Banach space theory, weak mixing and positive entropy reflect two of the basic regimes in which combinatorial independence can occur across the orbit of a tuple of sets in a dynamical system. The first of these asks for independence over a subset of the group having infinite cardinality, while the other requires this subset to satisfy a positive density condition. Inspired by work in the local theory of entropy [16], the authors

\footnotetext{
D. Kerr

Department of Mathematics, Texas A\&M University, College Station, TX 77843-3368, USA

e-mail: kerr@math.tamu.edu

H. Li (凶)

Department of Mathematics, Chongqing University, Chongqing 401331, China e-mail: hfli@math.buffalo.edu

H. Li

Department of Mathematics, SUNY at Buffalo, Buffalo, NY 14260-2900, USA
} 
studied this connection between independence, weak mixing, and entropy in [21, 22] as part of a program to develop a general theory of combinatorial independence in dynamics.

Combinatorial independence is the basic set-theoretic expression of randomness in which we are concerned not with the size of intersections, as in the probabilistic context, but merely with their nonemptiness. A collection $\left\{\left(A_{i, 1}, \ldots, A_{i, k}\right)\right\}_{i \in J}$ of $k$ tuples of subsets of a set $X$ is independent if for every nonempty finite set $F \subseteq J$ and function $\omega: F \rightarrow\{1, \ldots, k\}$ the intersection $\bigcap_{i \in F} A_{i, \omega(i)}$ is nonempty. If a group $G$ is acting on $X$ then given a tuple $\left(A_{1}, \ldots, A_{k}\right)$ of subsets of $X$ we say that a set $J \subseteq G$ is an independence set for $\left(A_{1}, \ldots, A_{k}\right)$ if the collection $\left\{\left(s^{-1} A_{1}, \ldots, s^{-1} A_{k}\right)\right\}_{s \in J}$ is independent. In the case of an action of a countable amenable group $G$ on a compact Hausdorff space $X$, the independence density $I(\boldsymbol{A})$ of a tuple $\boldsymbol{A}=\left(A_{1}, \ldots, A_{k}\right)$ of subsets of $X$ is defined as the limit of $\varphi_{A}(F) /|F|$ as the nonempty finite set $F \subseteq G$ becomes more and more left invariant, where $\varphi_{A}(F)$ denotes the maximum of the cardinalities of the independence sets for $\boldsymbol{A}$ which are contained in $F$ [21, Proposition 3.23]. We then say that a tuple $\left(x_{1}, \ldots, x_{k}\right) \in X^{k}$ is an IE-tuple if for every product neighborhood $U_{1} \times \cdots \times U_{k}$ of $\left(x_{1}, \ldots, x_{k}\right)$ the tuple $\boldsymbol{U}=\left(U_{1}, \ldots, U_{k}\right)$ satisfies $I(\boldsymbol{U})>0$. This condition on $\boldsymbol{U}$ is equivalent to the existence of an independence set $J \subseteq G$ for $\boldsymbol{U}$ which has positive density with respect to a given tempered Følner sequence $\left\{F_{i}\right\}_{i \in \mathbb{N}}$ in the sense that $\lim _{i \rightarrow \infty}\left|F_{i} \cap J\right| /\left|F_{i}\right|>0$. It turns out that a nondiagonal tuple is an IE-tuple if and only if it is an entropy tuple [21, Sect. 3]. For general acting $G$ we define IT-tuples in the same way as IE-tuples except that we ask instead for the independence set to have infinite cardinality. Versions of IE-tuples and IT-tuples can also be defined for probability-measure-preserving actions by requiring the independence condition on the set $J$ to hold whenever we remove small parts of the sets $s^{-1} U_{1}, \ldots, s^{-1} U_{k}$ for each $s \in G$. The following are some of the results relating independence, weak mixing, and entropy that were established in [21, 22].

(1) A continuous action $G \curvearrowright X$ of an Abelian group on a compact Hausdorff space is (topologically) weakly mixing if and only if every tuple of points in $X$ is an IT-tuple (in which case the action is said to be uniformly untame of all orders).

(2) A probability-measure-preserving action $G \curvearrowright(X, \mu)$ of an arbitrary group is weakly mixing if and only if its universal topological model is uniformly untame of all orders.

(3) A continuous action $G \curvearrowright X$ of a discrete amenable group on a compact Hausdorff space has positive topological entropy if and only if it has a nondiagonal IE-pair (for $G=\mathbb{Z}$ this was first proved by Huang and Ye in [18] using measuretheoretic techniques). Moreover, the action has uniformly positive entropy if and only if every pair of points in $X$ is an IE-pair, and uniformly positive entropy of all orders if and only if every tuple of points in $X$ is an IE-tuple.

(4) A probability-measure-preserving action $G \curvearrowright(X, \mu)$ of a discrete amenable group has positive measure entropy if and only if there is a nondiagonal measure IE-pair in some topological model. Moreover, the action has complete positive entropy if and only if every tuple of points is an IE-tuple in the universal topological model (for $G=\mathbb{Z}$ this was proved by Glasner and Weiss in [15]).

Chung and Li applied IE-tuples in [7] as part of a new approach for studying the relation between homoclinicity and entropy in expansive algebraic actions that enabled 
them to break the commutativity barrier and establish some duality-type equivalences for polycyclic-by-finite acting groups. In this case, and more generally for actions of a countable amenable group on a compact group $X$ by automorphisms, the analysis of IE-tuples is governed by a single closed invariant normal subgroup of $X$ called the IE group [7, Sect. 7].

Recent seminal work of Bowen in [5] has expanded the scope of the classical theory of entropy for actions of discrete amenable groups to the much broader realm of sofic acting groups. For a countable group $G$, soficity can be expressed as the existence of a sequence $\Sigma=\left\{\sigma_{i}: G \rightarrow \operatorname{Sym}\left(d_{i}\right)\right\}$ of maps from $G$ into finite permutation groups which is asymptotically multiplicative and free in the sense that

(i) $\lim _{i \rightarrow \infty} \frac{1}{d_{i}}\left|\left\{k \in\left\{1, \ldots, d_{i}\right\}: \sigma_{i, s t}(k)=\sigma_{i, s} \sigma_{i, t}(k)\right\}\right|=1 \quad$ for all $s, t \in G, \quad$ and

(ii) $\lim _{i \rightarrow \infty} \frac{1}{d_{i}}\left|\left\{k \in\left\{1, \ldots, d_{i}\right\}: \sigma_{i, s}(k) \neq \sigma_{i, t}(k)\right\}\right|=1 \quad$ for all distinct $s, t \in G$.

Such a sequence for which $\lim _{i \rightarrow \infty} d_{i}=\infty$ we call a sofic approximation sequence. By measuring the asymptotic exponential growth of dynamical models which are compatible with a fixed sofic approximation sequence, Bowen defined in [5] a collection of invariants for probability-measure-preserving actions of a countable sofic group admitting a generating partition with finite Shannon entropy. A remarkable application of this sofic measure entropy was a far-reaching extension of the OrnsteinWeiss classification of Bernoulli actions of amenable groups.

The authors developed in [23] a more general operator-algebraic approach to sofic entropy that enables one to remove the generator hypothesis (see also [20] for a formulation in terms of finite partitions). This led to a sofic version of topological entropy as well as a variational principle relating it to sofic measure entropy. We used this variational principle to compute the sofic topological entropy of a principal algebraic action $G \curvearrowright \mathbb{Z} \widehat{G / \mathbb{Z} G} f$ of a countable residually finite group in the case that the sofic approximation sequence arises from finite quotients of $G$ and $f$ is invertible in the full group $C^{*}$-algebra $C^{*}(G)$. In line with previous work on algebraic actions $[6,8,26,31]$ (see also [27]), this value turns out to be equal to the logarithm of the Fuglede-Kadison determinant of $f$ in the group von Neumann algebra $\mathcal{L} G$. We also showed how topological entropy can be used to give a proof that Gottschalk's surjunctivity conjecture holds for countable sofic groups, a result originally established by Gromov in [17], where the idea of soficity itself first appeared.

In the present work we initiate a local analysis of independence as it connects to topological entropy within this broadened framework of actions of sofic groups. Given a continuous action $G \curvearrowright X$ of a countable sofic group and a sofic approximation sequence $\Sigma=\left\{\sigma_{i}: G \rightarrow \operatorname{Sym}\left(d_{i}\right)\right\}$ for $G$, we define the notion of a $\Sigma$-IEtuple by externalizing the positive independence density condition in the amenable case to the finite sets $\left\{1, \ldots, d_{i}\right\}$ appearing in the sequence $\Sigma$ (Definition 4.3). We show in Sect. 4 that $\Sigma$-IE-tuples share many of the same properties as IE-tuples for actions of discrete amenable groups. In particular, the action $G \curvearrowright X$ has positive entropy with respect to $\Sigma$ if and only if there is a nondiagonal $\Sigma$-IE-pair in 
$X \times X$. On the other hand, we do not know whether the product formula holds in general for $\Sigma$-IE-tuples. However, granted that we use a free ultrafilter $\mathfrak{F}$ over $\mathbb{N}$ to express the independence density condition in the definition of $\Sigma$-IE-tuples, we demonstrate in Theorem 5.2 that the product formula holds under the assumption of ergodicity on the action of the commutant of $G$ inside the group of measurepreserving automorphisms of the Loeb space $\prod_{\mathfrak{F}}\left\{1, \ldots, d_{i}\right\}$ which arise from permutations of the sets $\left\{1, \ldots, d_{i}\right\}$. We then prove that this commutant acts ergodically when $G$ is residually finite and $\Sigma$ is built from finite quotients of $G$ (Theorem 5.7), and also when $G$ is amenable and $\Sigma$ is arbitrary (Theorem 5.8). In the case that $G$ is nonamenable, a combination of results of Elek and Szabo [12, Theorem 2] and Paunescu [35] shows that there exist $\Sigma$ for which the ergodicity condition fails.

The definition of IE-tuples for amenable $G$, as given in [21], involves an asymptotic density condition over finite subsets of $G$ which become more and more invariant. Although density in this sense loses its meaning in the nonamenable case, we might nevertheless ask what the external independence density in the definition of $\Sigma$-IE-tuples implies about the degree of independent behavior across orbits in $X$. We observe in Proposition 4.6 that every $\Sigma$-IE-tuple (and more generally every sofic IE-tuple as defined in Definition 4.3) is an orbit IE-tuple, by which we mean that for every product neighborhood $U_{1} \times \cdots \times U_{k}$ of the given tuple $\left(x_{1}, \ldots, x_{k}\right)$ in $X^{k}$, the tuple $\left(U_{1}, \ldots, U_{k}\right)$ has positive independence density over $G$ in the sense that there is a $q>0$ such that every finite set $F \subseteq G$ has a subset of cardinality at least $q|F|$ which is an independence set for $\left(U_{1}, \ldots, U_{k}\right)$ (note that this definition makes sense for any acting group $G$ ). We show moreover in Theorem 4.8 that, for amenable $G, \Sigma$-IE-tuples, IE-tuples, and orbit IE-tuples are all the same thing. This puts us in the pleasant and somewhat surprising situation that IE-tuples can be identified by a density condition that does not structurally depend on amenability for its formulation, and raises the question about the relation between entropy and orbit IE-tuples for nonamenable sofic $G$. In another direction, Theorem 7.1 asserts that if a tuple of subsets of $X$ has positive independence density over $G$ then it has an infinite independence set in $G$, which implies that every orbit IE-tuple is an IT-tuple.

By a theorem of Chung and $\mathrm{Li}$ in [7], an algebraic action of a countable group $G$ is expansive if and only if it is either the dual action $\left.G \curvearrowright X_{A}:=(\mathbb{Z} G)^{n /(\mathbb{Z}} G\right)^{n} A$ for some $n \in \mathbb{N}$ and matrix $A \in M_{n}(\mathbb{Z} G)$ which is invertible in $M_{n}\left(\ell^{1}(G)\right)$, or the restriction of such an action to a closed $G$-invariant subgroup of $X_{A}$. In the same paper it is shown that an expansive algebraic action $G \curvearrowright X$ of a polycyclic-by-finite group has completely positive entropy with respect to the Haar measure precisely when the IE group is equal to $X$, which is equivalent to every tuple of points in $X$ being an IE-tuple. It is also shown that, when $G$ is amenable, every action of the form $G \curvearrowright X_{A}$ with $A$ invertible in $M_{n}\left(\ell^{1}(G)\right)$ has the property that every tuple of points in $X$ is an IE-tuple (see Lemma 5.4 and Theorems 7.3 and 7.8 in [7]). We prove in Theorem 6.7 that if $G$ is a countable sofic group, $n \in \mathbb{N}$, and $A$ is a matrix in $M_{n}(\mathbb{Z} G)$ which is invertible in $M_{n}\left(\ell^{1}(G)\right)$, then the algebraic action $G \curvearrowright X_{A}$ has the property that every tuple of points in $X_{A}$ is a $\Sigma$-IE-tuple for every sofic approximation sequence $\Sigma$. We use this to answer a question of Deninger in the case 
that $G$ is residually finite by combining it with an argument from [7] and the entropy computation for principal algebraic actions from [23] mentioned above to deduce that if $f$ is an element of $\mathbb{Z} G$ which is invertible in $\ell^{1}(G)$ and has no left inverse in $\mathbb{Z} G$ then the Fuglede-Kadison determinant of $f$ satisfies $\operatorname{det}_{\mathcal{L} G} f>1$ (Corollary 6.8). Deninger asked whether this holds for all countable groups [9, Question 26], and affirmative answers were given in [10] for residually finite amenable $G$ and more generally in [7] for amenable $G$.

For a continuous action $G \curvearrowright X$ of a countably infinite group on a compact metrizable space with compatible metric $\rho$, we say that a pair $(x, y) \in X \times X$ is a Li-Yorke pair if

$$
\limsup _{G \ni s \rightarrow \infty} \rho(s x, s y)>0 \quad \text { and } \quad \liminf _{G \ni s \rightarrow \infty} \rho(s x, s y)=0
$$

where the limit supremum and limit infimum mean the limits of $\sup _{s \in G \backslash F} \rho(s x, s y)$ and $\inf _{s \in G \backslash F} \rho(s x, s y)$, respectively, over the net of finite subsets $F$ of $G$. Note that the definition of Li-Yorke pair does not depend on the choice of the metric $\rho$. The action $G \curvearrowright X$ is said to be Li-Yorke chaotic if there is an uncountable subset $Z$ of $X$ such that every nondiagonal pair $(x, y)$ in $Z \times Z$ is a Li-Yorke pair. The notion of Li-Yorke chaos stems from [28]. In the case of a continuous map $T: X \rightarrow X$, a theorem of Blanchard et al. in [3] states that positive entropy implies Li-Yorke chaos. In [21] the authors strengthened this by showing that for every $k \geq 2$ and product neighborhood $U_{1} \times \cdots \times U_{k}$ of a nondiagonal IE-tuple $\left(x_{1}, \ldots, x_{k}\right) \in X^{k}$ there are Cantor sets $Z_{i} \subseteq U_{i}$ for $i=1, \ldots, k$ such that

(1) every nonempty tuple of points in $\bigcup_{i} Z_{i}$ is an IE-tuple, and

(2) for all $m \in \mathbb{N}$, distinct $y_{1}, \ldots, y_{m} \in \bigcup_{i} Z_{i}$, and $y_{1}^{\prime}, \ldots, y_{m}^{\prime} \in \bigcup_{i} Z_{i}$ one has

$$
\liminf _{n \rightarrow \infty} \max _{1 \leq i \leq m} \rho\left(T^{n} y_{i}, y_{i}^{\prime}\right)=0
$$

In Theorem 8.1 we show that a similar result holds when $G$ is sofic and IE-tuples are replaced by $\Sigma$-IE-tuples as defined with respect to a free ultrafilter $\mathfrak{F}$ on $\mathbb{N}$, where $\Sigma$ is any sofic approximation sequence for $G$. Using $\mathfrak{F}$ in the definition of entropy, we deduce that if the action has positive entropy for some $\Sigma$ then it is Li-Yorke chaotic. As a corollary, if the action $G \curvearrowright X$ is distal then $h_{\Sigma}(X, G)=0$ or $-\infty$. In particular, when $G$ is amenable every distal action $G \curvearrowright X$ has zero entropy, which is well known in the case $G=\mathbb{Z}$ [34].

The following diagram illustrates how some of the main results of the paper relate various properties of actions of a countable discrete group $G$ on a compact metrizable space $X$, which we assume to have more than one point. In the left column we assume that $G$ is sofic and that $\Sigma$ is a fixed but arbitrary sofic approximation sequence. The unlabeled implications are trivial. By pair we mean an element of $X \times X$. See [21] for terminology related to tameness and nullness. 


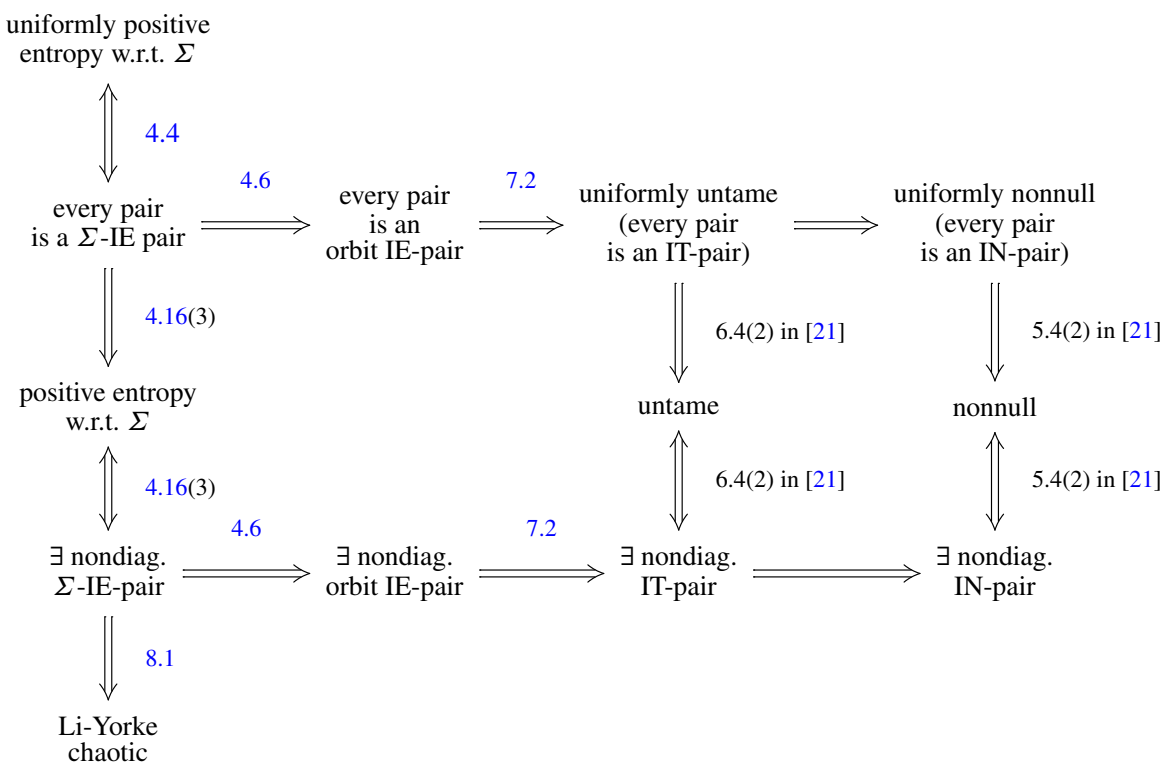

The organization of the paper is as follows. In Sect. 2 we set up some basic notation and review sofic topological entropy. In Sect. 3 we introduce orbit IE-tuples and prove a product formula for them. Section 4 introduces $\Sigma$-IE-tuples and includes our results relating them to orbit IE-tuples. In Sect. 5 we focus on the product formula for $\Sigma$-IE-tuples and the question of ergodicity for the action of $G^{\prime}$ on the Loeb space. Section 6 contains the material on algebraic actions. In Sect. 7 we prove that positive independence density for a tuple of subsets implies the existence of an infinite independence set, showing that orbit IE-tuples are IT-tuples. Finally in Sect. 8 we establish the theorem connecting independence and entropy to Li-Yorke chaos in the sofic framework.

\section{Sofic Topological Entropy}

We review the definition of sofic topological entropy $[23,24]$ and in the process introduce some of the basic notation and terminology appearing throughout the paper. Our approach will bypass the operator algebra technology that appears in [23, 24].

Let $Y$ be a set equipped with a pseudometric $\rho$ and let $\varepsilon \geq 0$. A set $A \subseteq Y$ is said to be $(\rho, \varepsilon)$-separated if $\rho(x, y) \geq \varepsilon$ for all distinct $x, y \in A$. Write $N_{\varepsilon}(Y, \rho)$ for the maximum cardinality of a $(\rho, \varepsilon)$-separated subset of $Y$.

Let $G \curvearrowright X$ be a continuous action of a countable sofic group on a compact metrizable space. Let $\Sigma=\left\{\sigma_{i}: G \rightarrow \operatorname{Sym}\left(d_{i}\right)\right\}$ be a sofic approximation sequence for $G$, meaning that

(i) $\lim _{i \rightarrow \infty} \frac{1}{d_{i}}\left|\left\{k \in\left\{1, \ldots, d_{i}\right\}: \sigma_{i, s t}(k)=\sigma_{i, s} \sigma_{i, t}(k)\right\}\right|=1 \quad$ for all $s, t \in G$,

(ii) $\lim _{i \rightarrow \infty} \frac{1}{d_{i}}\left|\left\{k \in\left\{1, \ldots, d_{i}\right\}: \sigma_{i, s}(k) \neq \sigma_{i, t}(k)\right\}\right|=1 \quad$ for all distinct $s, t \in G$, 
and $d_{i} \rightarrow \infty$ as $i \rightarrow \infty$. Depending on the situation, for $a \in\left\{1, \ldots, d_{i}\right\}$ we may write $\sigma_{i, s}(a), \sigma_{i}(s) a$, or $s a$ to denote the image of $a$ under the evaluation of $\sigma_{i}$ at $s$.

Let $\rho$ be a continuous pseudometric on $X$. For a given $d \in \mathbb{N}$, we define on the set of all maps from $\{1, \ldots, d\}$ to $X$ the pseudometrics

$$
\begin{aligned}
\rho_{2}(\varphi, \psi) & =\left(\frac{1}{d} \sum_{a=1}^{d}(\rho(\varphi(a), \psi(a)))^{2}\right)^{1 / 2}, \\
\rho_{\infty}(\varphi, \psi) & =\max _{a=1, \ldots, d} \rho(\varphi(a), \psi(a)) .
\end{aligned}
$$

Definition 2.1 Let $F$ be a nonempty finite subset of $G$ and $\delta>0$. Let $\sigma$ be a map from $G$ to $\operatorname{Sym}(d)$ for some $d \in \mathbb{N}$. Define $\operatorname{Map}(\rho, F, \delta, \sigma)$ to be the set of all maps $\varphi:\{1, \ldots, d\} \rightarrow X$ such that $\rho_{2}\left(\varphi \sigma_{s}, \alpha_{s} \varphi\right)<\delta$ for all $s \in F$, where $\alpha_{s}$ is the transformation $x \mapsto s x$ of $X$.

Definition 2.2 Let $F$ be a nonempty finite subset of $G$ and $\delta>0$. For $\varepsilon>0$ define

$$
\begin{aligned}
h_{\Sigma, 2}^{\varepsilon}(\rho, F, \delta) & =\limsup _{i \rightarrow \infty} \frac{1}{d_{i}} \log N_{\varepsilon}\left(\operatorname{Map}\left(\rho, F, \delta, \sigma_{i}\right), \rho_{2}\right), \\
h_{\Sigma, 2}^{\varepsilon}(\rho, F) & =\inf _{\delta>0}^{\varepsilon} h_{\Sigma, 2}^{\varepsilon}(\rho, F, \delta), \\
h_{\Sigma, 2}^{\varepsilon}(\rho) & =\inf _{F} h_{\Sigma, 2}^{\varepsilon}(\rho, F), \\
h_{\Sigma, 2}(\rho) & =\sup _{\varepsilon>0} h_{\Sigma, 2}^{\varepsilon}(\rho),
\end{aligned}
$$

where $F$ in the third line ranges over the nonempty finite subsets of $G$. In the case that $\operatorname{Map}\left(\rho, F, \delta, \sigma_{i}\right)$ is empty for all sufficiently large $i$, we set $h_{\Sigma, 2}^{\varepsilon}(\rho, F, \delta)=-\infty$. We similarly define $h_{\Sigma, \infty}^{\varepsilon}(\rho, F, \delta), h_{\Sigma, \infty}^{\varepsilon}(\rho, F), h_{\Sigma, \infty}^{\varepsilon}(\rho)$ and $h_{\Sigma, \infty}(\rho)$ using $N_{\varepsilon}\left(\cdot, \rho_{\infty}\right)$ in place of $N_{\varepsilon}\left(\cdot, \rho_{2}\right)$.

Instead of the limit supremum above we could have taken a limit over a fixed free ultrafilter on $\mathbb{N}$, whose utility is apparent for example if we wish to have a product formula (see Sect. 5). We will also use this variant in Sect. 8.

The pseudometric $\rho$ is said to be dynamically generating if for every pair of distinct points $x, y \in X$ there is an $s \in G$ such that $\rho(s x, s y)>0$.

Lemma 2.3 Suppose that $\rho$ and $\rho^{\prime}$ are continuous pseudometrics on $X$ and that $\rho^{\prime}$ is dynamically generating. Let $F$ be a nonempty finite subset of $G$ and $\delta>0$. Then there exist a nonempty finite subset $F^{\prime}$ of $G$ and $\delta^{\prime}>0$ such that for any $d \in \mathbb{N}$ and sufficiently good sofic approximation $\sigma: G \rightarrow \operatorname{Sym}(d)$ one has $\operatorname{Map}\left(\rho^{\prime}, F^{\prime}, \delta^{\prime}, \sigma\right) \subseteq$ $\operatorname{Map}(\rho, F, \delta, \sigma)$. 
Proof List the elements of $G$ as $s_{1}, s_{2}, \ldots$ Since $\rho^{\prime}$ is dynamically generating, we have the compatible metric $\rho^{\prime \prime}$ on $X$ defined by

$$
\rho^{\prime \prime}(x, y)=\sum_{k=1}^{\infty} \frac{1}{2^{k}} \rho^{\prime}\left(s_{k} x, s_{k} y\right) .
$$

It follows that there are a nonempty finite subset $F^{\prime \prime}$ of $G$ and a $\delta^{\prime \prime}>0$ such that, for all $x, y \in X$, if $\max _{s \in F^{\prime \prime}} \rho^{\prime}(s x, s y)<\delta^{\prime \prime}$ then $\rho(x, y)<\delta / 2$. Set $F^{\prime}=$ $F^{\prime \prime} \cup\left(F^{\prime \prime} F\right)$. Let $\delta^{\prime}>0$ and let $\sigma$ be a map from $G$ to $\operatorname{Sym}(d)$ for some $d \in \mathbb{N}$. Let $\varphi \in \operatorname{Map}\left(\rho^{\prime}, F^{\prime}, \delta^{\prime}, \sigma\right)$. Then

$$
\begin{aligned}
\mid\{a \in & \{1, \ldots, d\}: \rho^{\prime}\left(s_{1} s_{2} \varphi(a), \varphi\left(\left(s_{1} s_{2}\right) a\right)\right)<\sqrt{\delta^{\prime}} \text { and } \\
& \left.\rho^{\prime}\left(s_{1} \varphi\left(s_{2} a\right), \varphi\left(s_{1}\left(s_{2} a\right)\right)\right)<\sqrt{\delta^{\prime}} \text { for all } s_{1} \in F^{\prime \prime}, s_{2} \in F\right\} \mid \\
\geq & \left(1-2\left|F^{\prime \prime}\right||F| \delta^{\prime}\right) d .
\end{aligned}
$$

Suppose that $2 \sqrt{\delta^{\prime}}<\delta^{\prime \prime}$ and $\sigma$ is a good enough sofic approximation for $G$ so that

$$
\mid\left\{a \in\{1, \ldots, d\}:\left(s_{1} s_{2}\right) a=s_{1}\left(s_{2} a\right) \text { for all } s_{1} \in F^{\prime \prime}, s_{2} \in F\right\} \mid \geq\left(1-\delta^{\prime}\right) d .
$$

Then

$$
\begin{aligned}
& \mid\{a \in\{1, \ldots, d\}: \rho(s \varphi(a), \varphi(s a))<\delta / 2 \text { for all } s \in F\} \mid \\
& \quad \geq \mid\left\{a \in\{1, \ldots, d\}: \rho^{\prime}\left(s_{1} s_{2} \varphi(a), s_{1} \varphi\left(s_{2} a\right)\right)<2 \sqrt{\delta^{\prime}} \text { for all } s_{1} \in F^{\prime \prime}, s_{2} \in F\right\} \mid \\
& \quad \geq\left(1-\left(1+2\left|F^{\prime \prime}\right||F|\right) \delta^{\prime}\right) d .
\end{aligned}
$$

It follows that when $\delta^{\prime}$ is small enough independently of $d$ and $\sigma$, one has $\varphi \in$ $\operatorname{Map}(\rho, F, \delta, \sigma)$.

The following proposition is contained in Proposition 2.4 of [24], whose statement and proof use the operator-algebraic formulation of sofic topological entropy from [23].

Proposition 2.4 Let $\rho$ and $\rho^{\prime}$ be continuous pseudometrics on $X$ which are dynamically generating. Then

$$
h_{\Sigma, 2}(\rho)=h_{\Sigma, 2}\left(\rho^{\prime}\right)=h_{\Sigma, \infty}(\rho)=h_{\Sigma, \infty}\left(\rho^{\prime}\right)
$$

Proof Since the pseudometric $\rho_{\infty}$ dominates the pseudometric $\rho_{2}$, we have $h_{\Sigma, 2}(\rho) \leq h_{\Sigma, \infty}(\rho)$.

Next, we argue that $h_{\Sigma, \infty}(\rho) \leq h_{\Sigma, 2}(\rho)$. Let $F$ be a finite subset of $G, \delta>0$, and $\sigma$ a map from $G$ to $\operatorname{Sym}(d)$ for some $d \in \mathbb{N}$. Let $1 / 2>\varepsilon>0$. Let $\eta>0$ be the minimum of $\varepsilon^{2}$ and the reciprocal of the minimum cardinality of an $(\varepsilon / 2)$-spanning subset of $X$ with respect to $\rho$. Given a $\varphi \in \operatorname{Map}(\rho, F, \delta, \sigma)$, every element in the open $\left(\rho_{2}, \eta\right)$-ball in $\operatorname{Map}(\rho, F, \delta, \sigma)$ around $\varphi$ agrees with $\varphi$ to within $\sqrt{\eta}$, and hence 
to within $\varepsilon$, on a subset of $\{1, \ldots, d\}$ of cardinality at least $(1-\eta) d$. Thus the maximum cardinality of a $\left(\rho_{\infty}, \varepsilon\right)$-separated subset of the open $\left(\rho_{2}, \eta\right)$-ball around $\varphi$ is at most $\sum_{j=0}^{\lfloor\eta d\rfloor}\left(\begin{array}{c}d \\ j\end{array}\right) \eta^{-j}$, which by Stirling's approximation is bounded above, for all $d$ sufficiently large, by $e^{\beta d} \eta^{-\eta d}$ for some $\beta>0$ not depending on $d$ with $\beta \rightarrow 0$ as $\varepsilon \rightarrow 0$. Hence

$$
N_{\varepsilon}\left(\operatorname{Map}(\rho, F, \delta, \sigma), \rho_{\infty}\right) \leq e^{\beta d} \eta^{-\eta d} N_{\eta}\left(\operatorname{Map}(\rho, F, \delta, \sigma), \rho_{2}\right) .
$$

It follows that

$$
h_{\Sigma, \infty}^{\varepsilon}(\rho) \leq h_{\Sigma, 2}^{\eta}(\rho)+\beta-\eta \log \eta,
$$

and since $\beta-\eta \log \eta \rightarrow 0$ as $\varepsilon \rightarrow 0$ we conclude that $h_{\Sigma, \infty}(\rho) \leq h_{\Sigma, 2}(\rho)$.

Finally we show that $h_{\Sigma, 2}(\rho) \leq h_{\Sigma, 2}\left(\rho^{\prime}\right)$, which will establish the proposition as we can interchange the roles of $\rho$ and $\rho^{\prime}$.

Let $\varepsilon>0$. Since $\rho$ is dynamically generating, we can find a finite set $K \subseteq G$ and an $\varepsilon^{\prime}>0$ such that, for all $x, y \in X$, if $\rho(s x, s y)<\sqrt{3 \varepsilon^{\prime}}$ for all $s \in K$ then $\rho^{\prime}(x, y)<$ $\varepsilon / \sqrt{2}$. By shrinking $\varepsilon^{\prime}$ if necessary we may assume that $3 \varepsilon^{\prime}|K|<\varepsilon^{2} / 2$. Take a finite set $F \subseteq G$ containing $K$ and a $\delta>0$ with $\delta \leq \varepsilon^{\prime}$ such that $h_{\Sigma, 2}^{\varepsilon^{\prime}}(\rho, F, \delta) \leq h_{\Sigma, 2}^{\varepsilon^{\prime}}(\rho)+$ $\varepsilon$. Since $\rho^{\prime}$ is dynamically generating, by Lemma 2.3 there are a nonempty finite set $F^{\prime} \subseteq G$ and a $\delta^{\prime}>0$ such that for any $d \in \mathbb{N}$ and sufficiently good sofic approximation $\sigma: G \rightarrow \operatorname{Sym}(d)$ we have $\operatorname{Map}\left(\rho^{\prime}, F^{\prime}, \delta^{\prime}, \sigma\right) \subseteq \operatorname{Map}(\rho, F, \delta, \sigma)$.

Given $\varphi, \psi \in \operatorname{Map}\left(\rho^{\prime}, F^{\prime}, \delta^{\prime}, \sigma\right)$ such that $\rho_{2}(\varphi, \psi)<\varepsilon^{\prime}$, for each $s \in K$ we have, writing $\alpha_{s}$ for the transformation $x \mapsto s x$ of $X$,

$$
\rho_{2}\left(\alpha_{s} \varphi, \alpha_{s} \psi\right) \leq \rho_{2}\left(\alpha_{s} \varphi, \varphi \sigma_{s}\right)+\rho_{2}\left(\varphi \sigma_{s}, \psi \sigma_{s}\right)+\rho_{2}\left(\psi \sigma_{s}, \alpha_{s} \psi\right)<\delta+\varepsilon^{\prime}+\delta \leq 3 \varepsilon^{\prime} .
$$

This implies that there is a set $W \subseteq\{1, \ldots, d\}$ of cardinality at least $\left(1-3 \varepsilon^{\prime}|K|\right) d$ such that for all $a \in W$ we have $\rho(s \varphi(a), s \psi(a))<\sqrt{3 \varepsilon^{\prime}}$ for every $s \in K$ and hence $\rho^{\prime}(\varphi(a), \psi(a))<\varepsilon / \sqrt{2}$. As a consequence, assuming (as we may by normalizing) that $X$ has $\rho^{\prime}$-diameter at most one,

$$
\rho_{2}^{\prime}(\varphi, \psi) \leq \sqrt{(\varepsilon / \sqrt{2})^{2}+3 \varepsilon^{\prime}|K|}<\varepsilon
$$

It follows that

$$
N_{\varepsilon}\left(\operatorname{Map}\left(\rho^{\prime}, F^{\prime}, \delta^{\prime}, \sigma\right), \rho_{2}^{\prime}\right) \leq N_{\mathcal{E}^{\prime}}\left(\operatorname{Map}(\rho, F, \delta, \sigma), \rho_{2}\right)
$$

and hence $h_{\Sigma, 2}^{\varepsilon}\left(\rho^{\prime}, F^{\prime}, \delta^{\prime}\right) \leq h_{\Sigma, 2}^{\varepsilon^{\prime}}(\rho, F, \delta)$, so that

$$
h_{\Sigma, 2}^{\varepsilon}\left(\rho^{\prime}\right) \leq h_{\Sigma, 2}^{\varepsilon}\left(\rho^{\prime}, F^{\prime}, \delta^{\prime}\right) \leq h_{\Sigma, 2}^{\varepsilon^{\prime}}(\rho, F, \delta) \leq h_{\Sigma, 2}^{\varepsilon^{\prime}}(\rho)+\varepsilon \leq h_{\Sigma, 2}(\rho)+\varepsilon .
$$

Since $\varepsilon$ was an arbitrary positive number we conclude that $h_{\Sigma, 2}\left(\rho^{\prime}\right) \leq h_{\Sigma, 2}(\rho)$.

Definition 2.5 The topological entropy $h_{\Sigma}(X, G)$ of the action $G \curvearrowright X$ with respect to $\Sigma$ is defined to be the common value in Proposition 2.4 over all dynamically generating continuous pseudometrics on $X$. 
Note that the approximate multiplicativity of a sofic approximation was only needed in the proof of Lemma 2.3 to handle the situation in which one of $\rho$ and $\rho^{\prime}$ is not an actual metric. Indeed we could have defined topological entropy more easily by using the obvious fact that $h_{\Sigma, \infty}(\rho)$ takes a common value over all compatible metrics on $X$, with Proposition 2.4 then being regarded as a Kolmogorov-Sinai theorem. As with the $(n, \varepsilon)$-separated set definition of topological entropy for single transformations, it is by considering pseudometrics that we can compute the entropy for a nontrivial example like the shift action $G \curvearrowright\{1, \ldots, k\}^{G}$. In this case one can see that the value is $\log k$ independently of $\Sigma$ by considering the pseudometric $\rho$ on $\{1, \ldots, k\}^{G}$ given by $\rho(x, y)=0$ or 1 depending on whether or not the coordinates of $x$ and $y$ at $e$ agree. Indeed $\log k$ is easily seen to be an upper bound, and given a nonempty finite set $F \subseteq G$, a $\delta>0$, and a good enough sofic approximation $\sigma: G \rightarrow \operatorname{Sym}(d)$ we can construct a $\left(\rho_{\infty}, 1 / 2\right)$-separated subset of $\operatorname{Map}(\rho, F, \delta, \sigma)$ of cardinality $k^{d}$ by associating to every $\omega \in\{1, \ldots, k\}^{d}$ some $\varphi_{\omega} \in \operatorname{Map}(\rho, F, \delta, \sigma)$ defined by $\varphi_{\omega}(a)\left(s^{-1}\right)=\omega\left(\sigma_{s}(a)\right)$ for all $a \in\{1, \ldots, d\}$ and $s \in G$.

For actions of amenable $G$, the entropy $h_{\Sigma}(X, G)$ coincides with the classical topological entropy for every $\Sigma$ [23]. Such an action always has a largest zeroentropy factor (i.e., a zero-entropy factor such that every zero-entropy factor factors through it), called the topological Pinsker factor [4]. More generally for sofic $G$, with respect to a fixed $\Sigma$ there exists a largest factor of the action $G \curvearrowright X$ which has entropy either 0 or $-\infty$ (note that the value $-\infty$ does not occur for actions of amenable $G$ ). This follows from the fact that the property of having entropy 0 or $-\infty$ is preserved under taking countable products and restricting to closed invariant sets. (The property of having entropy $-\infty$ is also preserved under taking countable products, though we do not know what happens to the property of having entropy 0 .) We say that the action has completely positive entropy with respect to $\Sigma$ if each of its nontrivial factors has positive entropy with respect to $\Sigma$.

Unlike in the amenable case, the largest factor with entropy 0 or $-\infty$ might have factors with positive entropy. In fact for every nonamenable $G$ there exist zeroentropy actions of $G$ which have factors with positive entropy: Take an action $G \curvearrowright X$ with $h_{\Sigma}(X, G)>0$ and an action $G \curvearrowright Y$ which has no $G$-invariant Borel probability measure, and consider the action of $G$ on $K:=(X \times Y) \bigsqcup\{z\}$ where $z$ is a point on which $G$ acts trivially. Then $h_{\Sigma}(K, G)=0$ but the quotient action on $X \coprod\{z\}$ satisfies $h_{\Sigma}(X \coprod\{z\}, G)>0$.

\section{Orbit IE-tuples}

Let $G \curvearrowright X$ be a continuous action of a discrete group on a compact Hausdorff space. Recall from the introduction that if $\boldsymbol{A}=\left(A_{1}, \ldots, A_{k}\right)$ is a tuple of subsets of $X$ then we say that a subset $F$ of $G$ is an independence set for $\boldsymbol{A}$ if for every finite subset $J$ of $F$ and every function $\omega: J \rightarrow\{1, \ldots, k\}$ we have $\bigcap_{s \in J} s^{-1} A_{\omega(s)} \neq \emptyset$.

Definition 3.1 Let $\boldsymbol{A}=\left(A_{1}, \ldots, A_{k}\right)$ be a tuple of subsets of $X$. We define the independence density of $\boldsymbol{A}$ (over $G$ ) to be the largest $q \geq 0$ such that every finite set $F \subseteq G$ has a subset of cardinality at least $q|F|$ which is an independence set for $\boldsymbol{A}$. 
Definition 3.2 We say that a tuple $\boldsymbol{x}=\left(x_{1}, \ldots, x_{k}\right) \in X^{k}$ is an orbit IE-tuple (or orbit IE-pair in the case $k=2$ ) if for every product neighborhood $U_{1} \times \cdots \times U_{k}$ of $\boldsymbol{x}$ the tuple $\left(U_{1}, \ldots, U_{k}\right)$ has positive independence density. Write $\operatorname{IE}_{k}(X, G)$ for the set of all orbit IE-tuples of length $k$.

As Theorem 4.8 below demonstrates, the notation $\operatorname{IE}_{k}(X, G)$ is consistent with its use in [21] when $G$ is amenable.

The equality in the next theorem statement is understood with respect to the identification of $\left(\left(x_{1}, \ldots, x_{k}\right),\left(y_{1}, \ldots, y_{k}\right)\right) \in X^{k} \times Y^{k}$ and $\left(\left(x_{1}, y_{1}\right), \ldots,\left(x_{k}, y_{k}\right)\right) \in$ $(X \times Y)^{k}$.

Theorem 3.3 Let $G \curvearrowright X$ and $G \curvearrowright Y$ be continuous actions on compact Hausdorff spaces. Let $k \in \mathbb{N}$. Then

$$
\operatorname{IE}_{k}(X \times Y, G)=\operatorname{IE}_{k}(X, G) \times \operatorname{IE}_{k}(Y, G) .
$$

Proof The inclusion $\operatorname{IE}_{k}(X \times Y, G) \subseteq \operatorname{IE}_{k}(X, G) \times \operatorname{IE}_{k}(Y, G)$ is trivial. To prove the other direction, it suffices to show that if $\boldsymbol{A}=\left(A_{1}, \ldots, A_{k}\right)$ is a tuple of subsets of $X$ with independence density $q$ and $\boldsymbol{B}=\left(B_{1}, \ldots, B_{k}\right)$ is a tuple of subsets of $Y$ with independence density $r$, then $\boldsymbol{A} \times \boldsymbol{B}:=\left(A_{1} \times B_{1}, \ldots, A_{k} \times B_{k}\right)$ has independence density at least $q r$. Let $F$ be a nonempty finite subset of $G$. Then we can find a $J \subseteq F$ with $|J| \geq q|F|$ which is an independence set for $\boldsymbol{A}$. We can then find a $J_{1} \subseteq J$ with $\left|J_{1}\right| \geq r|J|$ which as an independence set for $\boldsymbol{B}$. Then $J_{1}$ is an independence set for $\boldsymbol{A} \times \boldsymbol{B}$ and $\left|J_{1}\right| \geq q r|F|$.

In [21] we defined a tuple $\boldsymbol{x}=\left(x_{1}, \ldots, x_{k}\right) \in X^{k}$ to be an IN-tuple if for every product neighborhood $U_{1} \times \cdots \times U_{k}$ of $\boldsymbol{x}$ the tuple $\left(U_{1}, \ldots, U_{k}\right)$ has arbitrarily large finite independence sets. The following fact is obvious.

Proposition 3.4 Suppose that $G$ is infinite. Then every orbit IE-tuple is an IN-tuple.

We will strengthen this assertion in Theorem 7.1.

\section{$4 \Sigma$-IE-tuples}

Unless otherwise stated, throughout this section $G$ is a countable sofic discrete group, subject to further hypotheses as appropriate. We suppose $G$ to be acting continuously on a compact metrizable space $X$, and $\rho$ denotes a dynamically generating continuous pseudometric on $X$ unless otherwise stated.

In order to be able to define the notion of a sofic IE-tuple as appears in Proposition 4.6, we will set up our definitions for a general sofic approximation net $\Sigma=\left\{\sigma_{i}: G \rightarrow \operatorname{Sym}\left(d_{i}\right)\right\}$, which is formally defined in the same way as the sequential version.

Definition 4.1 Let $\boldsymbol{A}=\left(A_{1}, \ldots, A_{k}\right)$ be a tuple of subsets of $X$. Let $F$ be a nonempty finite subset of $G$ and $\delta>0$. Let $\sigma$ be a map from $G$ to $\operatorname{Sym}(d)$ for some $d \in \mathbb{N}$. 
We say that a set $\mathcal{J} \subseteq\{1, \ldots, d\}$ is a $(\rho, F, \delta, \sigma)$-independence set for $\boldsymbol{A}$ if for every function $\omega: \mathcal{J} \rightarrow\{1, \ldots, k\}$ there exists a $\varphi \in \operatorname{Map}(\rho, F, \delta, \sigma)$ such that $\varphi(a) \in A_{\omega(a)}$ for every $a \in \mathcal{J}$.

Definition 4.2 Let $\boldsymbol{A}=\left(A_{1}, \ldots, A_{k}\right)$ be a tuple of subsets of $X$. Let $\Sigma=\left\{\sigma_{i}: G \rightarrow\right.$ $\left.\operatorname{Sym}\left(d_{i}\right)\right\}$ be a sofic approximation net for $G$. We say that $\boldsymbol{A}$ has positive upper independence density over $\Sigma$ if there exists a $q>0$ such that for every nonempty finite set $F \subseteq G$ and $\delta>0$ there is a cofinal set of $i$ for which $\boldsymbol{A}$ has a $\left(\rho, F, \delta, \sigma_{i}\right)$ independence set of cardinality at least $q d_{i}$. By Lemma 2.3 this definition does not depend on the choice of $\rho$.

For the purposes of Sects. 5 and 8, we will consider a variation of the above definition in which cofinality is replaced by the stronger requirement of membership in a fixed free ultrafilter $\mathfrak{F}$ on $\mathbb{N}$. The resulting notion of positive upper independence density over $\Sigma$ with respect to $\mathfrak{F}$ will then be used when interpreting the following definition of $\Sigma$-IE-tuples.

By the universal sofic approximation net for $G$ we mean the net $(\sigma, F) \mapsto \sigma$ indexed by the directed set of pairs $(\sigma, F)$ where $\sigma$ is a map from $G$ to $\operatorname{Sym}(d)$ for some $d \in \mathbb{N}$ and $F$ is a finite subset of $G$, and $\left(\sigma^{\prime}: G \rightarrow \operatorname{Sym}\left(d^{\prime}\right), F^{\prime}\right) \succ$ $(\sigma: G \rightarrow \operatorname{Sym}(d), F)$ means that $d^{\prime} \geq d$ and $\left|V\left(\sigma^{\prime}, F\right)\right| / d^{\prime} \geq|V(\sigma, F)| / d$, where $V(\omega, F)$ for a map $\omega: G \rightarrow \operatorname{Sym}(c)$ denotes the set of all $a \in\{1, \ldots, c\}$ such that $\sigma(s) \sigma(t) a=\sigma(s t) a$ for all $s, t \in F$ and $\sigma(s) a \neq \sigma(t) a$ for all distinct $s, t \in F$.

Definition 4.3 Let $\Sigma=\left\{\sigma_{i}: G \rightarrow \operatorname{Sym}\left(d_{i}\right)\right\}$ be a sofic approximation net for $G$. We say that a tuple $\boldsymbol{x}=\left(x_{1}, \ldots, x_{k}\right) \in X^{k}$ is a $\Sigma$-IE-tuple (or $\Sigma$-IE-pair in the case $k=2$ ) if for every product neighborhood $U_{1} \times \cdots \times U_{k}$ of $\boldsymbol{x}$ the tuple $\left(U_{1}, \ldots, U_{k}\right)$ has positive upper independence density over $\Sigma$. We say that $\boldsymbol{x}$ is a sofic IE-tuple (or sofic IE-pair in the case $k=2$ ) if it is a $\Sigma$-IE-tuple for the universal sofic approximation net $\Sigma$. We denote the $\Sigma$-IE-tuples of length $k$ by $\operatorname{IE}_{k}^{\Sigma}(X, G)$ and the sofic IE-tuples of length $k$ by $\operatorname{IE}_{k}^{\text {sof }}(X, G)$.

Note that $\operatorname{IE}_{k}^{\Sigma}(X, G) \subseteq \operatorname{IE}_{k}^{\text {sof }}(X, G)$ for every sofic approximation net $\Sigma$.

We define $\Sigma$-IE-tuples and sofic IE-tuples of sets in the same way as for points above.

Remark 4.4 It follows from Lemma 3.3 of [21] that a nondiagonal tuple of points in $X$ is a $\Sigma$-IE-tuple if and only if it is a $\Sigma$-entropy tuple in the sense of Sect. 5 in [40]. In particular, if in analogy with the amenable case we define the action to have uniformly positive entropy with respect to $\Sigma$ when every nondiagonal pair in $X \times X$ is a $\Sigma$-entropy pair, then the action has this property precisely when every pair in $X \times X$ is a $\Sigma$-IE-pair.

We will need the following consequence of Karpovsky and Milman's generalization of the Sauer-Shelah lemma [19, 36, 38]. 
Lemma 4.5 ([19]) Given $k \geq 2$ and $\lambda>1$ there is a constant $c>0$ such that, for all $n \in \mathbb{N}$, if $S \subseteq\{1,2, \ldots, k\}^{\{1,2, \ldots, n\}}$ satisfies $|S| \geq((k-1) \lambda)^{n}$ then there is an $I \subseteq\{1,2, \ldots, n\}$ with $|I| \geq c n$ and $\left.S\right|_{I}=\{1,2, \ldots, k\}^{I}$.

Proposition 4.6 A sofic IE-tuple is an orbit IE-tuple.

Proof Fix a compatible metric $\rho$ on $X$. Let $\boldsymbol{x}=\left(x_{1}, \ldots, x_{k}\right)$ be an IE-tuple and $U_{1} \times$ $\cdots \times U_{k}$ a product neighborhood of $\boldsymbol{x}$. We will show that the tuple $\left(U_{1}, \ldots, U_{k}\right)$ has positive independence density over $G$.

Suppose first that $k>1$. Take $1<\lambda<\frac{k}{k-1}$. Then we have the constant $c>0$ in Lemma 4.5.

Let $V_{1} \times \cdots \times V_{k}$ be a product neighborhood of $\boldsymbol{x}$ such that for some $\kappa>0$ the $\kappa$-neighborhood of $V_{j}$ is contained in $U_{j}$ for all $1 \leq j \leq k$. Then there exists a $q>0$ such that for every nonempty finite subset $F$ of $G$ and $\delta>0$ there is a cofinal set of $i$ for which the tuple $\left(V_{1}, \ldots, V_{k}\right)$ has a $\left(\rho, F, \delta, \sigma_{i}\right)$-independence set $\mathcal{J}_{i}$ of cardinality at least $q d_{i}$.

Let $F$ be a nonempty finite subset of $G$. We will show $F$ has a subset of cardinality at least $(c q / 2)|F|$ which is an independence set for the tuple $\left(U_{1}, \ldots, U_{k}\right)$. Let $\delta$ be a small positive number to be determined in a moment.

Take an $i$ in the above cofinal set with $\left|\mathcal{W}_{i}\right| \geq(1-\delta) d$ for $\mathcal{W}_{i}:=\{a \in$ $\left\{1, \ldots, d_{i}\right\}: F \stackrel{\sigma_{i}(\cdot) a}{\rightarrow} \sigma_{i}(F) a$ is injective $\}$. For each $\omega: \mathcal{J}_{i} \rightarrow\{1, \ldots, k\}$, take a $\varphi_{\omega} \in$ $\operatorname{Map}(\rho, F, \delta, \sigma)$ such that $\varphi_{\omega}(a) \in V_{\omega(a)}$ for all $a \in \mathcal{J}_{i}$. Then $\mid\left\{a \in\left\{1, \ldots, d_{i}\right\}\right.$ : $\left.\rho\left(s \varphi_{\omega}(a), \varphi_{\omega}(s a)\right) \leq \delta^{1 / 2}\right\} \mid \geq(1-\delta) d_{i}$ for each $s \in F$ and hence $\left|\Lambda_{\omega}\right| \geq(1-$ $|F| \delta) d_{i}$ for

$$
\Lambda_{\omega}:=\left\{a \in\left\{1, \ldots, d_{i}\right\}: \rho\left(s \varphi_{\omega}(a), \varphi_{\omega}(s a)\right) \leq \delta^{1 / 2} \text { for all } s \in F\right\} .
$$

Set $n=|F|$. When $n \delta<1 / 2$, the number of subsets of $\{1, \ldots, d\}$ of cardinality no greater than $n \delta d$ is equal to $\sum_{j=0}^{\lfloor n \delta d\rfloor}\left(\begin{array}{l}d \\ j\end{array}\right)$, which is at most $n \delta d\left(\begin{array}{c}d \\ n \delta d\end{array}\right)$, which by Stirling's approximation is less than $\exp (\beta d)$ for some $\beta>0$ depending on $\delta$ and $n$ but not on $d$ when $d$ is sufficiently large with $\beta \rightarrow 0$ as $\delta \rightarrow 0$ for a fixed $n$. Thus when $\delta$ is small enough and $i$ is large enough, there is a subset $\Omega_{i}$ of $\{1, \ldots, k\} \mathcal{J}_{i}$ with $\left(\frac{k}{(k-1) \lambda}\right)^{q d_{i}}\left|\Omega_{i}\right| \geq k^{\left|\mathcal{J}_{i}\right|}$ such that the set $\Lambda_{\omega}$ is the same, say $\Theta_{i}$, for every $\omega \in \Omega_{i}$, and $\left|\Theta_{i}\right| / d_{i}>1-|F| \delta$. Then

$$
\left|\Omega_{i}\right| \geq k^{\left|\mathcal{J}_{i}\right|}\left(\frac{(k-1) \lambda}{k}\right)^{q d_{i}} \geq k^{\left|\mathcal{J}_{i}\right|}\left(\frac{(k-1) \lambda}{k}\right)^{\left|\mathcal{J}_{i}\right|}=((k-1) \lambda)^{\left|\mathcal{J}_{i}\right|} .
$$

By our choice of $c$, we can find a subset $\mathcal{J}_{i}^{\prime}$ of $\mathcal{J}_{i}$ with $\left|\mathcal{J}_{i}^{\prime}\right| \geq c\left|\mathcal{J}_{i}\right| \geq c q d_{i}$ such that every $\xi: \mathcal{J}_{i}^{\prime} \rightarrow\{1, \ldots, k\}$ extends to some $\omega \in \Omega_{i}$.

Writing $\zeta$ for the uniform probability measure on $\{1, \ldots, d\}$, we have

$$
\begin{aligned}
\int_{\mathcal{W}_{i} \cap \Theta_{i}} \sum_{s \in F} 1_{\mathcal{J}_{i}^{\prime}}(s a) d \zeta(a) & =\sum_{s \in F} \int_{\mathcal{W}_{i} \cap \Theta_{i}} 1_{\mathcal{J}_{i}^{\prime}}(s a) d \zeta(a) \geq \sum_{s \in F}\left(\frac{\left|\mathcal{J}_{i}^{\prime}\right|}{d_{i}}-(|F|+1) \delta\right) \\
& \geq(c q-(|F|+1) \delta)|F|,
\end{aligned}
$$


and hence $\sum_{s \in F} 1_{\mathcal{J}_{i}^{\prime}}\left(s a_{i}\right) \geq(c q-(|F|+1) \delta)|F|$ for some $a_{i} \in \mathcal{W}_{i} \cap \Theta_{i}$. Then $\left|J_{i}\right| \geq(c q-(|F|+1) \delta)|F|$ for $J_{i}:=\left\{s \in F: s a_{i} \in \mathcal{J}_{i}^{\prime}\right\}$.

We claim that $J_{i}$ is an independence set for the tuple $\left(U_{1}, \ldots, U_{k}\right)$ when $\delta<\kappa^{2}$. Let $f \in\{1, \ldots, k\}^{J_{i}}$. Since $a_{i} \in \mathcal{W}_{i}$, the map $J_{i} \stackrel{\sigma_{i}(\cdot) a_{i}}{\rightarrow} \sigma_{i}\left(J_{i}\right) a_{i}$ is bijective. Thus we can define $\xi^{\prime} \in\{1, \ldots, k\}^{\sigma\left(J_{i}\right) a_{i}}$ by $\xi^{\prime}\left(s a_{i}\right)=f(s)$ for $s \in J_{i}$. Extend $\xi^{\prime}$ to some $\xi \in$ $\{1, \ldots, k\}^{\mathcal{J}_{i}^{\prime}}$. Then we can extend $\xi$ to some $\omega \in \Omega_{i}$. For every $s \in J_{i}$, since $s a_{i} \in \mathcal{J}_{i}$ and $a_{i} \in \Theta_{i}=\Lambda_{\omega}$, we have $\varphi_{\omega}\left(s a_{i}\right) \in V_{\omega\left(s a_{i}\right)}=V_{f(s)}$ and $\rho\left(s \varphi_{\omega}\left(a_{i}\right), \varphi_{\omega}\left(s a_{i}\right)\right) \leq$ $\delta^{1 / 2}<\kappa$. By the choice of $\kappa$ we have $s \varphi_{\omega}\left(a_{i}\right) \in U_{f(s)}$. This proves our claim.

Taking $\delta$ to be small enough, we have $\left|J_{i}\right| \geq(c q-(|F|+1) \delta)|F| \geq(c q / 2)|F|$ as desired.

The case $k=1$ can be established by a simpler version of the above argument that considers only a single map of the form $\varphi_{\omega}$ and does not require the invocation of the constant $c$ from Lemma 4.5 and the associated use of Stirling's approximation.

For the remainder of this subsection, $\Sigma=\left\{\sigma_{i}: G \rightarrow \operatorname{Sym}\left(d_{i}\right)\right\}$ is a fixed but arbitrary sofic approximation sequence.

In the case that $G$ is amenable, the independence density $I(\boldsymbol{A})$ of a tuple $\boldsymbol{A}$ of subsets of $X$ was defined on [21, p. 887] as the limit of $\varphi_{A}(F) /|F|$ as the nonempty finite set $F \subseteq G$ becomes more and more left invariant, where $\varphi_{A}(F)$ denotes the maximum of the cardinalities of the independence sets for $\boldsymbol{A}$ which are contained in $F$. A tuple $\left(x_{1}, \ldots, x_{k}\right) \in X^{k}$ is an IE-tuple if for every product neighborhood $U_{1} \times \cdots \times U_{k}$ of $\left(x_{1}, \ldots, x_{k}\right)$ the independence density $I(\boldsymbol{U})$ of the tuple $\boldsymbol{U}=\left(U_{1}, \ldots, U_{k}\right)$ is positive.

To establish Theorem 4.8 we need the following version of the Rokhlin lemma for sofic approximations, which appears as Lemma 4.6 in [24]. For $\lambda \geq 0$, a collection of subsets of $\{1, \ldots, d\}$ is said to $\lambda$-cover $\{1, \ldots, d\}$ if its union has cardinality at least $\lambda d$.

Lemma 4.7 Let $G$ be a countable amenable discrete group. Let $0 \leq \tau<1$ and $0<$ $\eta<1$. Let $K$ be a nonempty finite subset of $G$ and $\delta>0$. Then there are an $\ell \in \mathbb{N}$, nonempty finite subsets $F_{1}, \ldots, F_{\ell}$ of $G$ with $\left|K F_{k} \backslash F_{k}\right|<\delta\left|F_{k}\right|$ and $\left|F_{k} K \backslash F_{k}\right|<$ $\delta\left|F_{k}\right|$ for all $k=1, \ldots, \ell$, a finite set $F \subseteq G$ containing $e$, and an $\eta^{\prime}>0$ such that, for every $d \in \mathbb{N}$, every map $\sigma: G \rightarrow \operatorname{Sym}(d)$ for which there is a set $B \subseteq\{1, \ldots, d\}$ satisfying $|B| \geq\left(1-\eta^{\prime}\right) d$ and

$$
\sigma_{s t}(a)=\sigma_{s} \sigma_{t}(a), \quad \sigma_{s}(a) \neq \sigma_{s^{\prime}}(a), \quad \sigma_{e}(a)=a
$$

for all $a \in B$ and $s, t, s^{\prime} \in F$ with $s \neq s^{\prime}$, and every set $V \subseteq\{1, \ldots, d\}$ with $|V| \geq$ $(1-\tau) d$, there exist $C_{1}, \ldots, C_{\ell} \subseteq V$ such that

(1) for every $k=1, \ldots, \ell$, the map $(s, c) \mapsto \sigma_{s}(c)$ from $F_{k} \times C_{k}$ to $\sigma\left(F_{k}\right) C_{k}$ is bijective,

(2) the family $\left\{\sigma\left(F_{1}\right) C_{1}, \ldots, \sigma\left(F_{\ell}\right) C_{\ell}\right\}$ is disjoint and $(1-\tau-\eta)$-covers $\{1, \ldots, d\}$.

Theorem 4.8 Suppose that $G$ is amenable. Then IE-tuples, orbit IE-tuples, $\Sigma$-IEtuples, and sofic IE-tuples are all the same thing. 
Proof By Proposition 4.6, sofic IE-tuples and $\Sigma$-IE-tuples are orbit IE-tuples. That orbit IE-tuples are IE-tuples is clear in view of the definition of the independence density $I(\boldsymbol{A})$ of a tuple $\boldsymbol{A}$ of subsets of $X$. It thus remains to show that IE-tuples are both sofic IE-tuples and $\Sigma$-IE-tuples.

To prove that IE-tuples are $\Sigma$-IE-tuples, it suffices to demonstrate that, given a tuple $\boldsymbol{U}=\left(U_{1}, \ldots, U_{k}\right)$ of subsets of $X$ with $I(\boldsymbol{U})>0$, the tuple $\boldsymbol{U}$ has positive upper independence density over $\Sigma$. Set $\lambda=I(\boldsymbol{U})>0$. Let $F$ be a nonempty finite subset of $G$ and $\delta>0$.

Let $\eta>0$, to be determined. By Lemma 4.7 we can find an $\ell \in \mathbb{N}$ and nonempty finite sets $F_{1}, \ldots, F_{\ell} \subseteq G$ such that (i) the sets $F_{1}, \ldots, F_{\ell}$ are sufficiently left invariant so that for each $i=1, \ldots, \ell$ there is a set $J_{i} \subseteq F_{i}$ which is an independence set for $\boldsymbol{U}$ and has cardinality at least $\lambda\left|F_{i}\right| / 2$, and (ii) for every good enough sofic approximation $\sigma: G \rightarrow \operatorname{Sym}(d)$ there exist $C_{1}, \ldots, C_{\ell} \subseteq\{1, \ldots, d\}$ satisfying the following:

(1) for every $i=1, \ldots, \ell$ and $c \in C_{i}$, the map $s \mapsto \sigma_{s}(c)$ from $F_{i}$ to $\sigma\left(F_{i}\right) c$ is bijective,

(2) the family of sets $\sigma\left(F_{i}\right) c$ for $i=1, \ldots, \ell$ and $c \in C_{i}$ is disjoint and $(1-\eta)$-covers $\{1, \ldots, d\}$.

Let $\sigma: G \rightarrow \operatorname{Sym}(d)$ be a sufficiently good sofic approximation for $G$ for some $d \in \mathbb{N}$. For every $h=\left(h_{1}, \ldots, h_{\ell}\right) \in \prod_{i=1}^{\ell} X^{C_{i}}$ take a map $\varphi_{h}:\{1, \ldots, d\} \rightarrow X$ such that

$$
\varphi_{h}(s c)=s\left(h_{i}(c)\right)
$$

for all $i \in\{1, \ldots, \ell\}, c \in C_{i}$, and $s \in F_{i}$. We may assume in our invocation of Lemma 4.7 above that the sets $F_{1}, \ldots, F_{\ell}$ are sufficiently left invariant so that, assuming that $\eta$ is sufficiently small and $\sigma$ is a sufficiently good sofic approximation, we have $\varphi_{h} \in \operatorname{Map}(\rho, F, \delta, \sigma)$ for every $h \in \prod_{i=1}^{\ell} X^{C_{i}}$. Write $\mathcal{J}$ for the subset $\bigcup_{i=1}^{\ell} \bigcup_{s \in J_{i}} \bigcup_{c \in C_{i}} \sigma_{s}(c)$ of $\{1, \ldots, d\}$. From (1) and (2) we obtain

$$
|\mathcal{J}|=\sum_{i=1}^{\ell}\left|J_{i}\right|\left|C_{i}\right| \geq \sum_{i=1}^{\ell} \frac{\lambda}{2}\left|F_{i}\right|\left|C_{i}\right| \geq \frac{\lambda}{2}(1-\eta) d \geq \frac{\lambda}{4} d
$$

assuming that $\eta \leq 1 / 2$. Now whenever we are given $\omega_{i} \in\{1, \ldots, k\}^{J_{i}}$ for $i=1, \ldots, \ell$ we can find, since each $J_{i}$ is an independence set for $\boldsymbol{U}$, an $h=\left(h_{1}, \ldots, h_{\ell}\right) \in$ $\prod_{i=1}^{\ell} X^{C_{i}}$ such that $s h_{i}(c) \in U_{\omega_{i}(s)}$ for all $i=1, \ldots, \ell$ and $s \in J_{i}$. The maps $\varphi_{h}$ for such $h$ then witness the fact that $\mathcal{J}$ is a $(\rho, F, \delta, \sigma)$-independence set for $\boldsymbol{U}$. It follows that $\boldsymbol{U}$ has positive upper independence density over $\Sigma$. Hence IE-tuples are $\Sigma$-IE-tuples.

The above argument also shows that IE-tuples are sofic IE-tuples, and so we are done.

One can also give the following direct proof that IE-tuples are orbit IE-tuples. It suffices to show that if $\boldsymbol{A}=\left(A_{1}, \ldots, A_{k}\right)$ is a tuple of subsets of $X$ and $q>0$ is such that for every nonempty finite subset $K$ of $G$ and $\varepsilon>0$ there exist a nonempty finite subset $F$ of $G$ with $|K F \backslash F| \leq \varepsilon|F|$ and a $J \subseteq F$ with $|J| \geq q|F|$ which is an independence set for $\boldsymbol{A}$, then the independence density of $\boldsymbol{A}$ over $G$ is at least $q$. 
Let $F_{1}$ be a nonempty finite subset of $G$. Let $1>\delta>0$. Take $\varepsilon>0$ be a small number which we shall determine in a moment. Then there exist a nonempty finite subset $F$ of $G$ with $\left|F_{1}^{-1} F \backslash F\right| \leq \varepsilon|F|$ and a $J \subseteq F$ with $|J| \geq q|F|$ which is an independence set for $\boldsymbol{A}$. Set $F^{\prime}=\left\{s \in F: F_{1}^{-1} s \subseteq F\right\}$. Taking $\varepsilon$ to be small enough, we have $\left|F^{\prime}\right| \geq(1-\delta)|F|$. Note that the function $\sum_{s \in F} 1_{F_{1} s}$ has value $\left|F_{1}\right|$ at every point of $F^{\prime}$. Thus

$$
\sum_{t \in J \cap F^{\prime}} \sum_{s \in F} 1_{F_{1} s}(t)=\left|J \cap F^{\prime}\right|\left|F_{1}\right|
$$

We also have

$$
\sum_{t \in J \cap F^{\prime}} \sum_{s \in F} 1_{F_{1} s}(t)=\sum_{s \in F} \sum_{t \in J \cap F^{\prime}} 1_{F_{1} s}(t)=\sum_{s \in F}\left|F_{1} s \cap\left(J \cap F^{\prime}\right)\right| .
$$

Therefore we can find an $s \in F$ with

$$
\left|F_{1} s \cap\left(J \cap F^{\prime}\right)\right| \geq \frac{\left|J \cap F^{\prime}\right|\left|F_{1}\right|}{|F|} \geq(q-\delta)\left|F_{1}\right| .
$$

Since $F_{1} \cap\left(J \cap F^{\prime}\right) s^{-1}$ is an independence set for $\boldsymbol{A}$, we deduce that $F_{1}$ has a subset of cardinality at least $(q-\delta)\left|F_{1}\right|$ which is an independence set for $\boldsymbol{A}$. Letting $\delta \rightarrow 0$, we get that $F_{1}$ has a subset of cardinality at least $q\left|F_{1}\right|$ which is an independence set for $\boldsymbol{A}$. Therefore the independence density $I(\boldsymbol{A})$ of $\boldsymbol{A}$ is at least $q$, and so we conclude that IE-tuples are orbit IE-tuples.

The surprising fact above is that IE-tuples are orbit IE-tuples in the amenable case. It is clear however for a Bernoulli action that all tuples are orbit IE-tuples. Notice also that the argument above works equally well if in the definition of $\Sigma$-IE-tuples we use positive upper independence density over $\Sigma$ with respect to a fixed free ultrafilter $\mathfrak{F}$.

Remark 4.9 The product formula for IE-tuples as defined in the amenable framework was established in Theorem 3.15 of [21] using a measure-theoretic argument. We can now combine Theorems 4.8 and 3.3 to obtain a combinatorial proof.

Remark 4.10 The proof of Theorem 4.8 shows that the independence density $I(\boldsymbol{A})$, as defined on [21, p. 887] and recalled before the theorem statement, coincides with the independence density defined in Definition 3.1. We may thus use the notation $I(\boldsymbol{A})$ without ambiguity to denote the more general independence density of Definition 3.1.

Remark 4.11 When $G$ is amenable, it is clear from the classical $(n, \varepsilon)$-separated set formulation of topological entropy that the entropy of an action $G \curvearrowright X$ is bounded below by the supremum of $I(\boldsymbol{A}) \log k$ over all pairs $(k, \boldsymbol{A})$ where $k \in \mathbb{N}$ and $\boldsymbol{A}$ is a $k$-tuple of pairwise disjoint closed subsets of $X$. For Bernoulli actions the two quantities are equal. In the nonamenable case, the entropy fails in general to be bounded below by $\sup _{(k, \boldsymbol{A})} I(\boldsymbol{A}) \log k$, where $I(\boldsymbol{A})$ is as defined in Remark 4.10. Indeed an example of Ornstein and Weiss [33, Appendix C] shows that the Bernoulli action 
$F_{2} \curvearrowright\{0,1\}^{F_{2}}$ over the free group on two generators has Bernoulli factors over arbitrarily large finite sets of symbols, in which case the supremum is infinite.

We next aim to establish some basic properties of $\Sigma$-IE-tuples in Proposition 4.16. From Lemma 3.6 of [21] we obtain:

Lemma 4.12 Let $k \in \mathbb{N}$. Then there is a constant $c>0$ depending only on $k$ with the following property. Let $\boldsymbol{A}=\left(A_{1}, \ldots, A_{k}\right)$ be a $k$-tuple of subsets of $X$ and suppose $A_{1}=A_{1,1} \cup A_{1,2}$. Let $F$ be a nonempty finite subset of $G$ and $\delta>0$. Let $\sigma$ be a map from $G$ to $\operatorname{Sym}(d)$ for some $d \in \mathbb{N}$. If a set $J \subseteq\{1, \ldots, d\}$ is a $(\rho, F, \delta, \sigma)$ independence set for $\boldsymbol{A}$, then there exists an $I \subseteq J$ such that $|I| \geq c|J|$ and $I$ is a $(\rho, F, \delta, \sigma)$-independence set for $\left(A_{1,1}, \ldots, A_{k}\right)$ or $\left(A_{1,2}, \ldots, A_{k}\right)$.

From Lemma 4.12 we get:

Lemma 4.13 Let $\boldsymbol{A}=\left(A_{1}, \ldots, A_{k}\right)$ be a $k$-tuple of subsets of $X$ which has positive upper independence density over $\Sigma$. Suppose that $A_{1}=A_{1,1} \cup A_{1,2}$. Then at least one of the tuples $\left(A_{1,1}, \ldots, A_{k}\right)$ and $\left(A_{1,2}, \ldots, A_{k}\right)$ has positive upper independence density over $\Sigma$.

Lemma 4.14 $h_{\Sigma}(X, G)>0$ if and only if there are disjoint closed subsets $A_{0}$ and $A_{1}$ of $X$ such that $\left(A_{0}, A_{1}\right)$ has positive upper independence density over $\Sigma$.

Proof Let $\rho$ be a compatible metric on $X$ with $\operatorname{diam}_{\rho}(X) \leq 1$. Then $h_{\Sigma, \infty}(\rho)=$ $h_{\Sigma}(X, G)$. The "if" part is obvious. So assume $h_{\Sigma, \infty}(\rho)>0$. Then $h_{\Sigma, \infty}^{6 \varepsilon}(\rho)>0$ for some $\varepsilon>0$. Set $c=h_{\Sigma, \infty}^{6 \varepsilon}(\rho) / 2$.

Take a finite $(\rho, 2 \varepsilon)$-dense subset $Z$ of $X$. Consider on $X$ the continuous pseudometrics $\rho^{z}$, for $z \in Z$, and $\rho^{\prime}$ given by

$$
\rho^{z}(x, y)=|\rho(x, z)-\rho(y, z)|, \quad \rho^{\prime}(x, y)=\max _{z \in Z} \rho^{z}(x, y) .
$$

Note that if $\rho(x, y) \geq 6 \varepsilon$ for some $x, y \in X$, then $\rho^{\prime}(x, y) \geq 2 \varepsilon$. It follows that if $d \in \mathbb{N}$ and $\varphi$ and $\psi$ are maps from $\{1, \ldots, d\}$ to $X$ with $\rho_{\infty}(\varphi, \psi) \geq 6 \varepsilon$, then $\rho_{\infty}^{\prime}(\varphi, \psi) \geq 2 \varepsilon$.

Take an increasing sequence $\left\{F_{n}\right\}_{n \in \mathbb{N}}$ of nonempty finite subsets of $G$ with union $G$ and a decreasing sequence $\left\{\delta_{n}\right\}_{n \in \mathbb{N}}$ of positive numbers converging to 0 . For each $n \in \mathbb{N}$, there is a cofinal set $I_{n}$ of $i$ for which one has $N_{6 \varepsilon}\left(\operatorname{Map}\left(\rho, F_{n}, \delta_{n}, \sigma_{i}\right), \rho_{\infty}\right) \geq$ $\exp \left(c d_{i}\right)$. Then $N_{2 \varepsilon}\left(\operatorname{Map}\left(\rho, F_{n}, \delta_{n}, \sigma_{i}\right), \rho_{\infty}^{\prime}\right) \geq \exp \left(c d_{i}\right)$ for all $i \in I_{n}$. For each $i \in I_{n}$ and $z \in Z$ take a $\left(\rho_{\infty}^{z}, \varepsilon\right)$-separated subset $W_{i, z}$ of $\operatorname{Map}\left(\rho, F_{n}, \delta_{n}, \sigma_{i}\right)$ of maximum cardinality. Then

$$
N_{2 \varepsilon}\left(\operatorname{Map}\left(\rho, F_{n}, \delta_{n}, \sigma_{i}\right), \rho_{\infty}^{\prime}\right) \leq \prod_{z \in Z}\left|W_{i, z}\right|=\prod_{z \in Z} N_{\varepsilon}\left(\operatorname{Map}\left(\rho, F_{n}, \delta_{n}, \sigma_{i}\right), \rho_{\infty}^{z}\right) .
$$

Thus $N_{\varepsilon}\left(\operatorname{Map}\left(\rho, F_{n}, \delta_{n}, \sigma_{i}\right), \rho_{\infty}^{z_{n, i}}\right) \geq \exp \left(c d_{i} /|Z|\right)$ for some $z_{n, i} \in Z$. Replacing $I_{n}$ by a confinal subset if necessary, we may assume that $z_{n, i}$ is the same, say $z_{n}$, for all 
$i \in I_{n}$. Passing to a subsequence of $\left\{\left(F_{n}, \delta_{n}\right)\right\}_{n \in \mathbb{N}}$ if necessary, we may assume that $z_{n}$ is the same, say $\mathfrak{z}$, for all $n \in \mathbb{N}$.

Note that if $W$ is a $\left(\rho_{\infty}^{\mathfrak{z}}, \varepsilon\right)$-separated subset of $\operatorname{Map}\left(\rho, F_{n}, \delta_{n}, \sigma_{i}\right)$, then the set $\{\rho(\mathfrak{z}, \cdot) \circ \varphi: \varphi \in W\}$ in $\ell_{\infty}^{d_{i}}$ is $\left(\|\cdot\|_{\infty}, \varepsilon\right)$-separated. By [15, Lemma 2.3], there are constants $c^{\prime}$ and $\delta>0$ depending only on $c /|Z|$ and $\varepsilon$ such that for every $n \in \mathbb{N}$ and large enough $i \in I_{n}$ there are a $t_{n, i} \in[0,1]$ and a subset $J_{n, i}$ of $\left\{1, \ldots, d_{i}\right\}$ with $\left|J_{n, i}\right| \geq c^{\prime} d_{i}$ so that for every $\omega: J_{n, i} \rightarrow\{0,1\}$ there are a $\varphi_{\omega} \in \operatorname{Map}\left(\rho, F_{n}, \delta_{n}, \sigma_{i}\right)$ such that for all $a \in J_{n, i}$ we have $\rho\left(\mathfrak{z}, \varphi_{\omega}(a)\right) \geq t_{n, i}+\delta$ or $\rho\left(\mathfrak{z}, \varphi_{\omega}(a)\right) \leq t_{n, i}-\delta$ depending on whether $\omega(a)=0$ or $\omega(a)=1$. Replacing $I_{n}$ by a confinal subset if necessary, we may assume that there is a $t_{n} \in[0,1]$ such that $\left|t_{n, i}-t_{n}\right|<\delta / 4$ for all $i \in I_{n}$. Replacing $\left\{F_{n}, \delta_{n}\right\}_{n \in \mathbb{N}}$ by a subsequence if necessary, we may assume that there is a $t \in[0,1]$ such that $\left|t_{n}-t\right|<\delta / 4$ for all $n \in \mathbb{N}$. Set $A_{0}=\{x \in X: \rho(\mathfrak{z}, x) \geq$ $t+\delta / 2\}$ and $A_{1}=\{x \in X: \rho(\mathfrak{z}, z) \leq t-\delta / 2\}$. Then for every $n \in \mathbb{N}$ and $i \in I_{n}$, the set $J_{n, i}$ is a $\left(\rho, F_{n}, \delta_{n}, \sigma_{i}\right)$-independence set for $\left(A_{0}, A_{1}\right)$. Thus $\left(A_{0}, A_{1}\right)$ has positive upper independence density over $\Sigma$.

The following is obvious.

Lemma 4.15 $h_{\Sigma}(X, G) \geq 0$ if and only if $X$ as a 1-tuple has positive upper independence density over $\Sigma$.

Proposition 4.16 The following are true:

(1) Let $\left(A_{1}, \ldots, A_{k}\right)$ be a tuple of closed subsets of $X$ which has positive upper independence density over $\Sigma$. Then there exists a $\Sigma$-IE-tuple $\left(x_{1}, \ldots, x_{k}\right)$ with $x_{j} \in A_{j}$ for all $1 \leq j \leq k$.

(2) $\operatorname{IE}_{1}^{\Sigma}(X, G)$ is nonempty if and only if $h_{\Sigma}(X, G) \geq 0$.

(3) $\operatorname{IE}_{2}^{\Sigma}(X, G) \backslash \Delta_{2}(X)$ is nonempty if and only if $h_{\Sigma}(X, G)>0$, where $\Delta_{2}(X)$ denotes the diagonal in $X^{2}$.

(4) $\operatorname{IE}_{k}^{\Sigma}(X, G)$ is a closed subset of $X^{k}$ which is invariant under the product action.

(5) Let $\pi:(X, G) \rightarrow(Y, G)$ be a factor map. Then $(\pi \times \cdots \times \pi)\left(\operatorname{IE}_{k}^{\Sigma}(X, G)\right) \subseteq$ $\mathrm{IE}_{k}^{\Sigma}(Y, G)$.

(6) Suppose that $Z$ is a closed $G$-invariant subset of $X$. Then $\operatorname{IE}_{k}^{\Sigma}(Z, G) \subseteq$ $\mathrm{IE}_{k}^{\Sigma}(X, G)$.

Proof Assertion (1) follows from Lemma 4.13 and a simple compactness argument. Assertion (2) follows from assertion (1) and Lemma 4.15. Assertion (3) follows directly from assertion (1) and Lemma 4.14. Assertion (4) follows from the observation that, given a compatible metric $\rho$ of $X$, for any $s \in G$, nonempty finite subset $F$ of $G$, and $\delta>0$ there is a $\delta^{\prime}>0$ such that, for every $d \in \mathbb{N}$ and map $\sigma: G \rightarrow \operatorname{Sym}(d)$ which is a good enough sofic approximation for $G$, if $\varphi \in \operatorname{Map}\left(\rho,\left\{s^{-1}\right\} \cup\left(s^{-1} F\right), \delta^{\prime}, \sigma\right)$, then $\alpha_{s} \circ \varphi \circ \sigma_{s^{-1}} \in \operatorname{Map}(\rho, F, \delta, \sigma)$, where $\alpha_{s}$ is the transformation $x \mapsto s x$ of $X$. Assertions (5) and (6) are trivial.

Remark 4.17 The inclusion in (5) above is an equality when $G$ is amenable, since $\Sigma$ IE-tuples are the same as IE-tuples by Theorem 4.8. Equality can fail however if $G$ 
is nonamenable: Take an action $G \curvearrowright X$ with $h_{\Sigma}(X, G)=-\infty$ and an action $G \curvearrowright Y$ with $h_{\Sigma}(Y, G)>0$. Then $G \curvearrowright Y$ has a nondiagonal $\Sigma$-IE-pair, while the product action $G \curvearrowright X \times Y$, which factors onto $G \curvearrowright Y$ via the second coordinate projection, satisfies $h_{\Sigma}(X \times Y, G)=-\infty$ and hence has no nondiagonal $\Sigma$-IE-pairs.

Remark 4.18 The analogue for orbit IE-tuples of the localization in Proposition 4.16(1) does not hold in the nonamenable case. Indeed for any action $G \curvearrowright X$ of a discrete group the 1-tuple $X$ has positive independence density, while the boundary action $F_{2} \curvearrowright \partial F_{2}$ of the free group on two generators (where $\partial F_{2}$ consists of infinite reduced words in the standard generators and their inverses, with the action by left concatenation and reduction) is easily seen not to admit any orbit IE-1-tuples.

From Proposition 4.16(5) we get the following. As in Theorem 3.3, the inclusion below is understood with respect to the identification of $\left(\left(x_{1}, \ldots, x_{k}\right),\left(y_{1}, \ldots, y_{k}\right)\right) \in$ $X^{k} \times Y^{k}$ and $\left(\left(x_{1}, y_{1}\right), \ldots,\left(x_{k}, y_{k}\right)\right) \in(X \times Y)^{k}$.

Proposition 4.19 $\mathrm{IE}_{k}^{\Sigma}(X \times Y, G) \subseteq \operatorname{IE}_{k}^{\Sigma}(X, G) \times \operatorname{IE}_{k}^{\Sigma}(Y, G)$.

The problem of the reverse inclusion will be taken up in the next section.

For the remainder of this section $X$ is the unit ball of $\ell^{p}(G)$ for some $1 \leq p<\infty$ equipped with the pointwise convergence topology, and the action $G \curvearrowright X$ is by left shifts. We will use some of the above results to compute the sofic topological entropy of this action to be zero when $G$ is infinite.

Recall from the end of Sect. 3 that a tuple $\boldsymbol{x}=\left(x_{1}, \ldots, x_{k}\right) \in X^{k}$ is an IN-tuple if for every product neighborhood $U_{1} \times \cdots \times U_{k}$ of $\boldsymbol{x}$ the tuple $\left(U_{1}, \ldots, U_{k}\right)$ has arbitrarily large finite independence sets. We write $\operatorname{IN}_{k}(X, G)$ for the set of IN-tuples of length $k$.

Lemma 4.20 For every $k \in \mathbb{N}$ the set $\operatorname{IN}_{k}(X, G)$ consists of the single element $(0, \ldots, 0)$.

Proof Clearly $(0, \ldots, 0) \in \mathrm{IN}_{k}(X)$ for every $k \in \mathbb{N}$. Also note that if $\boldsymbol{x}=\left(x_{1}, \ldots\right.$, $\left.x_{k}\right) \in \mathrm{IN}_{k}(X)$ then $x_{1}, \ldots, x_{k} \in \mathrm{IN}_{1}(X)$. Thus it suffices to show $\operatorname{IN}_{1}(X) \subseteq\{0\}$.

Let $x \in X$ with $x \neq 0$. Then $(t x)_{e} \neq 0$ for some $t \in G$. Set $r=\left|(t x)_{e}\right| / 2>0$ and $U=\left\{y \in X:\left|y_{e}\right| \geq r\right\}$. Then $U$ is a neighborhood of $t x$ in $X$. Let $F \subseteq G$ be a finite independence set for $U$. Then $\bigcap_{s \in F} s^{-1} U$ is nonempty. Take $y \in \bigcap_{s \in F} s^{-1} U$. Then $s y \in U$ and hence $\left|y_{s^{-1}}\right|=\left|(s y)_{e}\right| \geq r$ for every $s \in F$. It follows that

$$
|F| r^{p} \leq \sum_{s \in F}\left|y_{s^{-1}}\right|^{p} \leq\|y\|_{p}^{p} \leq 1,
$$

and hence $|F| \leq r^{-p}$. Therefore $t x \notin \operatorname{IN}_{1}(X)$. Since $\operatorname{IN}_{1}(X)$ is $G$-invariant, $x \notin$ $\mathrm{IN}_{1}(X)$.

Proposition 4.21 Suppose that $G$ is infinite. Then $h_{\Sigma}(X, G)=0$. 
Proof By Lemma 4.20, Propositions 4.16(2), and Propositions 4.6 and 3.4, we have $h_{\Sigma}(X, G) \leq 0$. Since $X$ has the fixed point 0 , we have $h_{\Sigma}(X, G) \geq 0$. Therefore $h_{\Sigma}(X, G)=0$.

\section{Product Formula for IE-tuples}

In order to hope for a product formula for $\Sigma$-IE-tuples beyond the amenable case, we must be able to witness independence density in some uniform way, in analogy with the definition of orbit IE-tuples in Sect. 3 (see Theorem 3.3). This can be achieved by taking a free ultrafilter $\mathfrak{F}$ on $\mathbb{N}$ and requiring that the independence sets in Definition 4.2 exist for a set of $i$ belonging to $\mathfrak{F}$ instead a cofinal set of $i$. Thus for the purposes of this section we fix a free ultrafilter $\mathfrak{F}$ on $\mathbb{N}$ and switch to definition of $\Sigma$ IE-tuples based on this interpretation of positive density. We will similarly understand sofic topological entropy to be defined by using an ultralimit over $\mathfrak{F}$ in Definition 2.5 instead of the limit supremum. We do not know whether our product formula results, Proposition 5.1 and Theorem 5.2, hold for the original definitions.

For the first part of our discussion, up to and including Lemma 5.6, $G$ is a countable sofic group and $\Sigma=\left\{\sigma_{i}: G \rightarrow \operatorname{Sym}\left(d_{i}\right)\right\}_{i=1}^{\infty}$ a fixed but arbitrary sofic approximation sequence for $G$.

Proposition 5.1 Let $G$ act continuously on compact metrizable spaces $X$ and $Y$. Then

$$
h_{\Sigma}(X \times Y, G)=h_{\Sigma}(X, G)+h_{\Sigma}(Y, G) .
$$

Proof Fix compatible metrics $\rho^{X}$ and $\rho^{Y}$ on $X$ and $Y$ respectively. Define a compatible metric $\rho_{X \times Y}$ on $X \times Y$ by

$$
\rho^{X \times Y}\left(\left(x_{1}, y_{1}\right),\left(x_{2}, y_{2}\right)\right)=\rho^{X}\left(x_{1}, x_{2}\right)+\rho^{Y}\left(y_{1}, y_{2}\right)
$$

for $\left(x_{1}, y_{1}\right),\left(x_{2}, y_{2}\right) \in X \times Y$.

Let $d \in \mathbb{N}$. Identify $(X \times Y)^{\{1, \ldots, d\}}$ with $X^{\{1, \ldots, d\}} \times Y^{\{1, \ldots, d\}}$ naturally. Note that for all $\varphi, \varphi^{\prime} \in X^{\{1, \ldots, d\}}$ and $\psi, \psi^{\prime} \in Y^{\{1, \ldots, d\}}$ one has

$$
\max \left(\rho_{2}^{X}\left(\varphi, \varphi^{\prime}\right), \rho_{2}^{Y}\left(\psi, \psi^{\prime}\right)\right) \leq \rho_{2}^{X \times Y}\left((\varphi, \psi),\left(\varphi^{\prime}, \psi^{\prime}\right)\right) \leq \rho_{2}^{X}\left(\varphi, \varphi^{\prime}\right)+\rho_{2}^{Y}\left(\psi, \psi^{\prime}\right) .
$$

Let $F$ be a nonempty finite subset of $G, \delta>0$, and $\varepsilon>0$, and let $\sigma$ be a map from $G$ to $\operatorname{Sym}(d)$. Then $\operatorname{Map}\left(\rho^{X}, F, \delta, \sigma\right) \times \operatorname{Map}\left(\rho^{Y}, F, \delta, \sigma\right) \subseteq \operatorname{Map}\left(\rho^{X \times Y}, F, 2 \delta, \sigma\right)$. Furthermore, for every $\left(\rho_{2}^{X}, \varepsilon\right)$-separated subset $\mathcal{W}_{X}$ of $\operatorname{Map}\left(\rho^{X}, F, \delta, \sigma\right)$ and every $\left(\rho_{2}^{Y}, \varepsilon\right)$-separated subset $\mathcal{W}_{Y}$ of $\operatorname{Map}\left(\rho^{X}, F, \delta, \sigma\right)$, the set $\mathcal{W}_{X} \times \mathcal{W}_{Y}$ is $\left(\rho_{2}^{X \times Y}, \varepsilon\right)$ separated. It follows that $h_{\Sigma, 2}^{\varepsilon}\left(\rho^{X \times Y}, F, 2 \delta\right) \geq h_{\Sigma, 2}^{\varepsilon}\left(\rho^{X}, F, \delta\right)+h_{\Sigma, 2}^{\varepsilon}\left(\rho^{Y}, F, \delta\right)$, and hence $h_{\Sigma}(X \times Y, G) \geq h_{\Sigma}(X, G)+h_{\Sigma}(Y, G)$.

Note that for any $\left(\rho_{2}^{X}, \varepsilon\right)$-spanning subset $\mathcal{W}_{X}$ of $\operatorname{Map}\left(\rho^{X}, F, \delta, \sigma\right)$ and any $\left(\rho_{2}^{Y}, \varepsilon\right)$-spanning subset $\mathcal{W}_{Y}$ of $\operatorname{Map}\left(\rho^{X}, F, \delta, \sigma\right)$, the set $\mathcal{W}_{X} \times \mathcal{W}_{Y}$ is $\left(\rho_{2}^{X \times Y}, 2 \varepsilon\right)$ spanning for (though not necessarily contained in) $\operatorname{Map}\left(\rho^{X \times Y}, F, \delta, \sigma\right)$. It follows that $\quad N_{4 \varepsilon}\left(\rho^{X \times Y}, F, \delta, \sigma\right) \leq N_{\varepsilon}\left(\rho^{X}, F, \delta, \sigma\right) \quad \times \quad N_{\varepsilon}\left(\rho^{Y}, F, \delta, \sigma\right), \quad$ and 
hence $h_{\Sigma, 2}^{4 \varepsilon}\left(\rho^{X \times Y}, F, \delta\right) \leq h_{\Sigma, 2}^{\varepsilon}\left(\rho^{X}, F, \delta\right)+h_{\Sigma, 2}^{\varepsilon}\left(\rho^{Y}, F, \delta\right)$. Consequently, $h_{\Sigma}(X \times Y, G) \leq h_{\Sigma}(X, G)+h_{\Sigma}(Y, G)$.

The Loeb space and the Loeb measure were introduced by Loeb in [32]. An exposition can be found in [1]. The Loeb space is the ultraproduct space $\prod_{\mathfrak{F}}\left\{1, \ldots, d_{i}\right\}$. A subset $Y$ of $\prod_{\mathfrak{F}}\left\{1, \ldots, d_{i}\right\}$ is called internal if it is of the form $\prod_{\mathfrak{F}} Y_{i}$ for a sequence $\left\{Y_{i}\right\}_{i \in \mathbb{N}}$ with $Y_{i} \subseteq\left\{1, \ldots, d_{i}\right\}$ for all $i \in \mathbb{N}$. The collection $\mathfrak{I}$ of inner subsets is an algebra. The Loeb measure is the unique probability measure $\mu$ on the $\sigma$-algebra $\mathfrak{B}$ generated by $\mathfrak{I}$ such that $\mu(Y)=\lim _{i \rightarrow \mathfrak{F}}\left|\mathcal{Y}_{i}\right| / d_{i}$ for every internal set $Y=\prod_{\mathfrak{F}} \mathcal{Y}_{i}$. For every $Z \in \mathfrak{B}$ there exists a $Y \in \mathfrak{I}$ such that $\mu(Y \Delta Z)=0$.

For each $d \in \mathbb{N}$, denote by $\rho_{\text {Hamm }}$ the normalized Hamming distance on $\operatorname{Sym}(d)$ defined by

$$
\rho_{\mathrm{Hamm}}\left(\tau, \tau^{\prime}\right)=\frac{1}{d}\left|\left\{a \in\{1, \ldots, d\}: \tau(a) \neq \tau^{\prime}(a)\right\}\right| .
$$

The ultraproduct group $\prod_{\mathfrak{F}} \operatorname{Sym}\left(d_{i}\right)$ has a natural action on $\prod_{\mathfrak{F}}\left\{1, \ldots, d_{i}\right\}$ preserving $\mu$. One has a bi-invariant pseudometric $\rho_{L}$ on $\prod_{\mathfrak{F}} \operatorname{Sym}\left(d_{i}\right)$ defined by $\rho_{L}\left(\tau, \tau^{\prime}\right)=\mu\left(\left\{y \in \prod_{\mathfrak{F}}\left\{1, \ldots, d_{i}\right\}: \tau y \neq \tau^{\prime} y\right\}\right)$. For any $\tau=\left(\tau_{i}\right)_{i}, \tau^{\prime}=\left(\tau_{i}^{\prime}\right)_{i} \in$ $\prod_{\mathfrak{F}} \operatorname{Sym}\left(d_{i}\right)$ with $\tau_{i}, \tau_{i}^{\prime} \in \operatorname{Sym}\left(d_{i}\right)$ for all $i \in \mathbb{N}$, one has $\rho_{L}\left(\tau, \tau^{\prime}\right)=$ $\lim _{i \rightarrow \mathfrak{F}} \rho_{\text {Hamm }}\left(\tau_{i}, \tau_{i}^{\prime}\right)$. Denote by $\mathfrak{G}$ the quotient group of $\prod_{\mathfrak{F}} \operatorname{Sym}\left(d_{i}\right)$ by $\rho_{L}$. Then we may think of $\mathfrak{G}$ as acting on $\prod_{\mathfrak{F}}\left\{1, \ldots, d_{i}\right\}$ by $\mu$-preserving transformations.

The sofic approximation sequence $\Sigma$ gives rise to a natural group embedding of $G$ into $\mathfrak{G}$. Thus we may think of $G$ as a subgroup of $\mathfrak{G}$. Denote by $G^{\prime}$ the subgroup of $\mathfrak{G}$ consisting of elements commuting with $G$.

As before, the equality below is understood with respect to the identification of $\left(\left(x_{1}, \ldots, x_{k}\right),\left(y_{1}, \ldots, y_{k}\right)\right) \in X^{k} \times Y^{k}$ and $\left(\left(x_{1}, y_{1}\right), \ldots,\left(x_{k}, y_{k}\right)\right) \in(X \times Y)^{k}$.

Theorem 5.2 Suppose that the action of $G^{\prime}$ on $\left(\prod_{\mathfrak{F}}\left\{1, \ldots, d_{i}\right\}, \mathfrak{B}, \mu\right)$ is ergodic. Let $G$ act continuously on compact metrizable spaces $X$ and $Y$. Let $k \in \mathbb{N}$. Then

$$
\mathrm{IE}_{k}^{\Sigma}(X \times Y, G)=\operatorname{IE}_{k}^{\Sigma}(X, G) \times \operatorname{IE}_{k}^{\Sigma}(Y, G) .
$$

We prove the theorem by way of the following results.

Definition 5.3 Let $G$ act continuously on a compact metrizable space $X$. Let $\rho$ be a dynamically generating continuous pseudometric on $X$. Let $\boldsymbol{A}=\left(A_{1}, \ldots, A_{k}\right)$ be a tuple of subsets of $X$. We say that an internal set $Y=\prod_{\mathfrak{F}} Y_{i}$ with $Y_{i} \subseteq\left\{1, \ldots, d_{i}\right\}$ for all $i \in \mathbb{N}$ is an independence set for $\boldsymbol{A}$ if for every nonempty finite subset $F$ of $G$ and every $\delta>0$ the set of all $i \in \mathbb{N}$ for which $Y_{i}$ is a $\left(\rho, F, \delta, \sigma_{i}\right)$-independence set for $\boldsymbol{A}$ belongs to $\mathfrak{F}$.

From Lemma 2.3 it is easy to see that Definition 5.3 does not depend on the choice of $\rho$.

Consistent with our interpretation of the equality in Theorem 5.2, in Proposition 5.4 and Lemma 5.6 we understand $\boldsymbol{A} \times \boldsymbol{B}$ to mean $\left(A_{1} \times B_{1}, \ldots, A_{k} \times B_{k}\right)$ where $\boldsymbol{A}=\left(A_{1}, \ldots, A_{k}\right)$ and $\boldsymbol{B}=\left(B_{1}, \ldots, B_{k}\right)$. 
Proposition 5.4 Let $G$ act continuously on compact metrizable spaces $X$ and $Y$. Let $\boldsymbol{A}$ and $\boldsymbol{B}$ be $k$-tuples of subsets of $X$ and $Y$ respectively for some $k \in \mathbb{N}$. Then the following hold:

(1) A has positive upper independence density over $\Sigma$ if and only if $\boldsymbol{A}$ has an internal independence set $Z$ with $\mu(Z)>0$.

(2) The set of internal independence sets for $\boldsymbol{A}$ is $G^{\prime}$-invariant.

(3) An internal set is an independence set for $\boldsymbol{A} \times \boldsymbol{B}$ if and only if it in independence set for both $\boldsymbol{A}$ and $\boldsymbol{B}$.

Proof Fix a compatible metric $\rho$ on $X$ which gives $X$ diameter at most 1 .

(1) The "if" part is obvious. Suppose that $\boldsymbol{A}$ has positive upper independence density over $\Sigma$. Let $q>0$ be as in Definition 4.2. Let $\left\{F_{n}\right\}_{n \in \mathbb{N}}$ be an increasing sequence of finite subsets of $G$ with $\bigcup_{n \in \mathbb{N}} F_{n}=G$. For each $n \in \mathbb{N}$, denote by $W_{n}^{\prime}$ the set of all $i \in \mathbb{N}$ for which there is a $\left(\rho_{X}, F_{n}, 1 / n, \sigma_{i}\right)$-independence set $Z_{i}$ for $\boldsymbol{A}$ with $\zeta\left(Z_{i}\right) \geq q$. Also set $W_{n}=W_{n}^{\prime} \backslash\{1, \ldots, n-1\}$. Then $W_{n}^{\prime} \in \mathfrak{F}$ by our assumption and hence $W_{n} \in \mathfrak{F}$ for each $n \in \mathbb{N}$. Note that the sequence $\left\{W_{n}\right\}_{n \in \mathbb{N}}$ is decreasing, and $\bigcap_{n \in \mathbb{N}} W_{n}=\emptyset$.

We define an internal set $Z=\prod_{\mathfrak{F}} \mathcal{Z}_{i}$ as follows. If $i \in \mathbb{N} \backslash W_{1}$, we take any $\mathcal{Z}_{i} \subseteq$ $\left\{1, \ldots, d_{i}\right\}$. If $i \in W_{n} \backslash W_{n+1}$ for some $n \in \mathbb{N}$, we take $\mathcal{Z}_{i}$ to be a $\left(\rho, F_{n}, 1 / n, \sigma_{i}\right)$ independence set for $\boldsymbol{A}$ with $\left|\mathcal{Z}_{i}\right| / d_{i} \geq q$. Then $\mathcal{Z}_{i}$ is a $\left(\rho, F_{n}, 1 / n, \sigma_{i}\right)$-independence set for $\boldsymbol{A}$ for all $n \in \mathbb{N}$ and $i \in W_{n}$. Thus $Z$ is an internal independence set for $\boldsymbol{A}$. As $\left|\mathcal{Z}_{i}\right| / d_{i} \geq q$ for all $i \in W_{1}$, we have $\mu(Z)=\lim _{i \rightarrow \mathfrak{F}} \zeta\left(Z_{i}\right) \geq q$. This proves the "only if" part.

(2) Let $Z=\prod_{\mathfrak{F}} \mathcal{Z}_{i}$ be an internal independence set for $\boldsymbol{A}$, and let $\tau=\left(\tau_{i}\right)_{i} \in G^{\prime}$. Then $\tau Z=\prod_{\mathfrak{F}} \tau_{i} \mathcal{Z}_{i}$. Let $F$ be a nonempty finite subset of $G$ and $1>\delta>0$. Then the set $W$ of all $i \in \mathbb{N}$ for which $\mathcal{Z}_{i}$ is a $\left(\rho, F, \delta, \sigma_{i}\right)$-independence set for $\boldsymbol{A}$ is in $\mathfrak{F}$. Since $\tau \in G^{\prime}$, the set $V$ of all $i \in \mathbb{N}$ for which $\max _{s \in F} \rho_{\text {Hamm }}\left(\tau_{i}^{-1} \sigma_{i, s}, \sigma_{i, s} \tau_{i}^{-1}\right) \leq \delta^{2}$ is also in $\mathfrak{F}$. Then $V \cap W$ is in $\mathfrak{F}$. For every $i \in V, \varphi \in \operatorname{Map}\left(\rho, F, \delta, \sigma_{i}\right)$, and $s \in F$ one has

$$
\begin{aligned}
& \rho_{2}\left(\alpha_{s} \circ \varphi \circ \tau_{i}^{-1}, \varphi \circ \tau_{i}^{-1} \circ \sigma_{i, s}\right) \\
& \quad \leq \rho_{2}\left(\alpha_{s} \circ \varphi \circ \tau_{i}^{-1}, \varphi \circ \sigma_{i, s} \circ \tau_{i}^{-1}\right)+\rho_{2}\left(\varphi \circ \sigma_{i, s} \circ \tau_{i}^{-1}, \varphi \circ \tau_{i}^{-1} \circ \sigma_{i, s}\right) \\
& \quad \leq \rho_{2}\left(\alpha_{s} \circ \varphi, \varphi \circ \sigma_{i, s}\right)+\left(\rho_{\mathrm{Hamm}}\left(\tau_{i}^{-1} \circ \sigma_{i, s}, \sigma_{i, s} \circ \tau_{i}^{-1}\right)\right)^{1 / 2} \\
& \quad \leq \delta+\delta=2 \delta,
\end{aligned}
$$

where $\alpha_{s}$ is the transformation $x \mapsto s x$ of $X$, and hence $\varphi \circ \tau_{i}^{-1} \in \operatorname{Map}\left(\rho, F, 2 \delta, \sigma_{i}\right)$. It follows that for every $i \in V \cap W$ the set $\tau_{i} \mathcal{Z}_{i}$ is a $\left(\rho, F, 2 \delta, \sigma_{i}\right)$-independence set for $\boldsymbol{A}$. Therefore $\tau Z$ is an internal independence set for $\boldsymbol{A}$.

(3) This can be proved using arguments similar to the proof of Proposition 5.1.

Lemma 5.5 Suppose that $\Gamma$ is a subgroup of $\mathfrak{G}$ and the action of $\Gamma$ on $\left(\prod_{\mathfrak{F}}\{1, \ldots\right.$, $\left.\left.d_{i}\right\}, \mathfrak{B}, \mu\right)$ is ergodic. Let $Y, Z \in \mathfrak{B}$ be such that $\mu(Y), \mu(Z)>0$. Then $\mu(Y \cap \tau Z)>$ 0 for some $\tau \in \Gamma$. 
Proof Set $r=\sup _{F} \mu\left(\bigcup_{\tau \in F} \tau Z\right)$ with $F$ ranging over the nonempty countable subsets of $\Gamma$. Then we can find nonempty finite subsets $F_{1}, F_{2}, \ldots$ of $\Gamma$ such that $r=\lim _{n \rightarrow \infty} \mu\left(\bigcup_{\tau \in F_{n}} \tau Z\right)$. Set $W=\bigcup_{n \in \mathbb{N}} F_{n}$ and $Z^{\prime}=\bigcup_{\tau \in W} \tau Z$. Then $W$ is a countable subset of $\Gamma$ and $r=\mu\left(Z^{\prime}\right)$. For every $\tau^{\prime} \in \Gamma$ we have $\mu\left(Z \cup \tau^{\prime} Z\right)=$ $\mu\left(\bigcup_{\tau \in W \cup \tau^{\prime} W} \tau Z\right) \leq r$ and hence $\mu\left(\tau^{\prime} Z \backslash Z\right)=0$. Since the action of $\Gamma$ on $\left(\prod_{\mathfrak{F}}\left\{1, \ldots, d_{i}\right\}, \mathfrak{B}, \mu\right)$ is ergodic, we conclude that $r=1$. Thus $\mu(Y)=\mu\left(Y \cap Z^{\prime}\right) \leq$ $\sum_{\tau \in W} \mu(Y \cap \tau Z)$, and hence $\mu(Y \cap \tau Z)>0$ for some $\tau \in W$.

Lemma 5.6 Suppose that the action of $G^{\prime}$ on $\left(\prod_{\mathfrak{F}}\left\{1, \ldots, d_{i}\right\}, \mathfrak{B}, \mu\right)$ is ergodic. Let $G$ act continuously on compact metrizable spaces $X$ and $Y$. Let $k \in \mathbb{N}$ and let $\boldsymbol{A}$ and $\boldsymbol{B}$ be $k$-tuples of subsets of $X$ and $Y$, respectively. Suppose that both $\boldsymbol{A}$ and $\boldsymbol{B}$ have positive upper independence density over $\Sigma$. Then $\boldsymbol{A} \times \boldsymbol{B}$ also has positive upper independence density over $\Sigma$.

Proof This follows from Proposition 5.4 and Lemma 5.5.

Theorem 5.2 now follows from Proposition 4.19 and Lemma 5.6.

The remainder of this section is devoted to the problem of when the ergodicity hypothesis in Theorem 5.2 is satisfied. We prove that this happens when $G$ is residually finite and $\Sigma$ arises from finite quotients of $G$, and also when $G$ is amenable and $\Sigma$ is arbitrary. A combination of results of Elek and Szabo [12, Theorem 2] and Paunescu [35] shows on the other hand that if $G$ is nonamenable then there is always a sofic approximation sequence $\Sigma$ for which the commutant $G^{\prime}$ does not act ergodically.

Let $G$ be an infinite residually finite group, and let $\left\{G_{i}\right\}_{i \in \mathbb{N}}$ be a sequence of finite-index normal subgroups of $G$ such that $\bigcap_{n \in \mathbb{N}} \bigcup_{i \geq n} G_{i}=\{e\}$. Then we have the sofic approximation sequence $\Sigma=\left\{\sigma_{i}: G \rightarrow \operatorname{Sym}\left(\left|G / G_{i}\right|\right)\right\}$ by identifying $\left\{1, \ldots,\left|G / G_{i}\right|\right\}$ with $G / G_{i}$ and setting $\sigma_{i}(s)\left(t G_{i}\right)=$ st $G_{i}$ for $s, t \in G$.

Theorem 5.7 Under the above hypotheses, the action of $G^{\prime}$ on $\left(\prod_{\mathfrak{F}}\left\{1, \ldots,\left|G / G_{i}\right|\right\}\right.$, $\mathfrak{B}, \mu)$ is ergodic.

Proof Consider the right multiplication action $\sigma^{\prime}$ of $G / G_{i}$ on itself given by $\sigma_{i}^{\prime}\left(s G_{i}\right)\left(t G_{i}\right)=t s^{-1} G_{i}$ for $s, t \in G$. Since this commutes with $\sigma_{i}$, it suffices to show that the action of $\prod_{\mathfrak{F}} \sigma_{i}^{\prime}\left(G / G_{i}\right) \subseteq G^{\prime}$ on $\left(\prod_{\mathfrak{F}}\left\{1, \ldots,\left|G / G_{i}\right|\right\}, \mathfrak{B}, \mu\right)$ is ergodic.

Let $\mathcal{Y}_{i} \subseteq G / G_{i}$. Then, using the $\ell^{1}$-norm with respect to the uniform probability measure on $G / G_{i}$,

$$
\begin{aligned}
\frac{1}{\left|G / G_{i}\right|} \sum_{s G_{i} \in G / G_{i}}\left|\sigma_{i}^{\prime}\left(s G_{i}\right) \mathcal{Y}_{i} \Delta \mathcal{Y}_{i}\right| & =\sum_{s G_{i} \in G / G_{i}}\left\|1_{\sigma_{i}^{\prime}\left(s G_{i}\right) \mathcal{Y}_{i}}-1 \mathcal{Y}_{i}\right\|_{1} \\
& \geq\left\|\sum_{s G_{i} \in G / G_{i}} 1_{\sigma_{i}^{\prime}\left(s G_{i}\right) \mathcal{Y}_{i}}-\left|G / G_{i}\right| \cdot 1_{\mathcal{Y}_{i}}\right\|_{1} \\
& =\left\|\left|\mathcal{Y}_{i}\right| \cdot 1_{G / G_{i}}-\left|G / G_{i}\right| \cdot 1 \mathcal{Y}_{i}\right\|_{1} \\
& =2 \frac{\left|\mathcal{Y}_{i}\right|}{\left|G / G_{i}\right|}\left(\left|G / G_{i}\right|-\left|\mathcal{Y}_{i}\right|\right) .
\end{aligned}
$$


Thus there is some $s_{i} G_{i} \in G / G_{i}$ with $\frac{1}{\left|G / G_{i}\right|}\left|\sigma_{i}^{\prime}\left(s_{i} G_{i}\right) \mathcal{Y}_{i} \Delta \mathcal{Y}_{i}\right| \geq 2 \frac{\left|\mathcal{Y}_{i}\right|}{\left|G / G_{i}\right|}\left(1-\frac{\left|\mathcal{Y}_{i}\right|}{\left|G / G_{i}\right|}\right)$.

Let $Y=\prod_{\mathfrak{F}} \mathcal{Y}_{i}$ be an internal subset of $\prod_{\mathfrak{F}}\left\{1, \ldots,\left|G / G_{n}\right|\right\}$. Take $s_{i} G_{i} \in G / G_{i}$ as above for each $i \in \mathbb{N}$. Set $s=\left(s_{i} G_{i}\right)_{i}$. Then

$$
\begin{aligned}
\mu\left(\sigma^{\prime}(s) Y \Delta Y\right) & =\lim _{n \rightarrow \mathfrak{F}} \frac{\left|\sigma^{\prime}\left(s_{i} G_{i}\right) \mathcal{Y}_{i} \Delta \mathcal{Y}_{i}\right|}{\left|G / G_{i}\right|} \\
& \geq \lim _{n \rightarrow \mathfrak{F}} 2 \frac{\left|\mathcal{Y}_{i}\right|}{\left|G / G_{i}\right|}\left(1-\frac{\left|\mathcal{Y}_{i}\right|}{\left|G / G_{i}\right|}\right)=2 \mu(Y)(1-\mu(Y)) .
\end{aligned}
$$

If $\mu\left(\sigma^{\prime}(s) Y \Delta Y\right)=0$, then $\mu(Y)=0$ or 1 . This finishes the proof.

Theorem 5.8 Let $G$ be a countable amenable group. For every sofic approximation sequence $\Sigma$ for $G$, the action of $G^{\prime}$ on $\left(\prod_{\mathfrak{F}}\left\{1, \ldots, d_{i}\right\}, \mathfrak{B}, \mu\right)$ is ergodic.

The proof of Theorem 5.8 requires several lemmas.

We will use the following terminology. Let $(X, \mu)$ be a finite measure space and let $\delta \geq 0$. A family of measurable subsets of $X$ is said to $\delta$-cover $X$ if its union has measure at least $\delta \mu(X)$. A collection $\left\{A_{i}\right\}_{i \in I}$ of positive measure sets is $\delta$-disjoint if there exist pairwise disjoint sets $\widehat{A}_{i} \subseteq A_{i}$ such that $\mu\left(\widehat{A}_{i}\right) \geq(1-\delta) \mu\left(A_{i}\right)$ for all $i \in I$, and is a $\delta$-even covering of $X$ if there exists a number $M>0$ such that $\sum_{i \in I} \mathbf{1}_{A_{i}} \leq M$ and $\sum_{i \in I} \mu\left(A_{i}\right) \geq(1-\delta) M \mu(X)$.

The following is the Rokhlin lemma for sofic approximations, which is based on the quasitiling theory of Ornstein and Weiss and appears as Lemma 4.5 in [24]. The statement of the latter does not contain condition (3) below, but it is not hard to see from the proof in [24] that it can be arranged.

Lemma 5.9 Let $G$ be a countable discrete group. Let $0 \leq \theta<1$, and $0<\eta<1$. Then there are an $\ell^{\prime} \in \mathbb{N}$ and $\kappa, \eta^{\prime \prime}>0$ such that, whenever $e \in F_{1} \subseteq F_{2} \subseteq \cdots \subseteq F_{\ell^{\prime}}$ are finite subsets of $G$ with $\left|\left(F_{k-1}^{-1} F_{k}\right) \backslash F_{k}\right| \leq \kappa\left|F_{k}\right|$ for $k=2, \ldots, \ell^{\prime}$, there exist $\lambda_{1}, \ldots, \lambda_{\ell^{\prime}} \in[0,1]$ such that for every $\delta>0$, every sufficiently large $d \in \mathbb{N}$ (depending on $\delta)$, every map $\sigma: G \rightarrow \operatorname{Sym}(d)$ with a set $\mathcal{B} \subseteq\{1, \ldots, d\}$ satisfying $|\mathcal{B}| \geq\left(1-\eta^{\prime \prime}\right) d$ and

$$
\sigma_{s t}(a)=\sigma_{s} \sigma_{t}(a), \quad \sigma_{s}(a) \neq \sigma_{s^{\prime}}(a), \quad \sigma_{e}(a)=a
$$

for all $a \in \mathcal{B}$ and $s, t, s^{\prime} \in F_{\ell^{\prime}} \cup F_{\ell^{\prime}}^{-1}$ with $s \neq s^{\prime}$, and every set $\mathcal{V} \subseteq\{1, \ldots, d\}$ with $|\mathcal{V}| \geq(1-\theta) d$, there exist $\mathcal{C}_{1}, \ldots, \mathcal{C}_{\ell^{\prime}} \subseteq \mathcal{V}$ such that

(1) for every $k=1, \ldots, \ell^{\prime}$ and $c \in \mathcal{C}_{k}$, the map $s \mapsto \sigma_{s}(c)$ from $F_{k}$ to $\sigma\left(F_{k}\right) c$ is bijective,

(2) the sets $\sigma\left(F_{1}\right) \mathcal{C}_{1}, \ldots, \sigma\left(F_{\ell^{\prime}}\right) \mathcal{C}_{\ell^{\prime}}$ are pairwise disjoint and the family $\bigcup_{k=1}^{\ell^{\prime}}\left\{\sigma\left(F_{k}\right) c: c \in \mathcal{C}_{k}\right\}$ is $\eta$-disjoint and $(1-\theta-\eta)$-covers $\{1, \ldots, d\}$,

(3) $\sum_{k=1}^{\ell^{\prime}}|| \sigma\left(F_{k}\right) \mathcal{C}_{k}\left|/ d-\lambda_{k}\right|<\delta$.

Lemma 5.10 Let $G$ be a countable discrete group. Let $F$ be a nonempty finite subset of $G$. For every $d \in \mathbb{N}$, every map $\sigma: G \rightarrow \operatorname{Sym}(d)$, every set $\mathcal{B} \subseteq\{1, \ldots, d\}$ satisfying

$$
\sigma_{s}(a) \neq \sigma_{t}(a)
$$


for all $a \in \mathcal{B}$ and distinct $s, t \in F$, every $\mathcal{J} \subseteq\{1, \ldots, d\}$, and every $0<\lambda<1$, there exists $a \mathcal{V} \subseteq \mathcal{B}$ such that $|\mathcal{V}| \geq \frac{|\mathcal{B}|(1-\lambda)-d+|\mathcal{J}|}{1-\lambda}$ and $|\sigma(F) a \cap \mathcal{J}|>\lambda|F|$ for all $a \in \mathcal{V}$.

Proof Set $X=\{1, \ldots, d\}$. Denote by $\zeta$ the uniform probability measure on $X$. One has

$$
\begin{aligned}
\frac{1}{d} \sum_{a \in \mathcal{B}}|\sigma(F) a \cap \mathcal{J}| & =\int_{\mathcal{J}} \sum_{a \in \mathcal{B}} \mathbf{1}_{\sigma(F) a}(x) d \zeta(x) \\
& =\int_{X} \sum_{a \in \mathcal{B}} \mathbf{1}_{\sigma(F) a}(x) d \zeta(x)-\int_{X \backslash \mathcal{J}} \sum_{a \in \mathcal{B}} \mathbf{1}_{\sigma(F) a}(x) d \zeta(x) \\
& \geq \frac{|\mathcal{B}| \cdot|F|}{d}-\int_{X \backslash \mathcal{J}}|F| d \zeta(x) \\
& =\frac{|\mathcal{B}| \cdot|F|}{d}-\left(1-\frac{|\mathcal{J}|}{d}\right)|F| .
\end{aligned}
$$

Set $\mathcal{V}=\{a \in \mathcal{B}:|\sigma(F) a \cap \mathcal{J}|>\lambda|F|\}$. Then

$$
\frac{1}{d} \sum_{a \in \mathcal{B}}|\sigma(F) a \cap \mathcal{J}| \leq \frac{|\mathcal{V}| \cdot|F|}{d}+\frac{(|\mathcal{B}|-|\mathcal{V}|) \lambda|F|}{d}
$$

Thus

$$
\frac{|\mathcal{V}| \cdot|F|}{d}+\frac{(|\mathcal{B}|-|\mathcal{V}|) \lambda|F|}{d} \geq \frac{|\mathcal{B}| \cdot|F|}{d}-\left(1-\frac{|\mathcal{J}|}{d}\right)|F| .
$$

It follows that

$$
|\mathcal{V}| \geq \frac{|\mathcal{B}|(1-\lambda)-d+|\mathcal{J}|}{1-\lambda} .
$$

The proof of Lemma 4.4 in [24] shows the following.

Lemma 5.11 Let $(X, \mu)$ be a finite measure space. Let $\delta, \eta \in[0,1)$ and let $\left\{A_{i}\right\}_{i \in I}$ be a finite $\delta$-even covering of $X$ by positive measure sets. Then every $\eta$-disjoint subcollection of $\left\{A_{i}\right\}_{i \in I}$ can be enlarged to an $\eta$-disjoint subcollection of $\left\{A_{i}\right\}_{i \in I}$ which $\eta(1-\delta)$-covers $X$.

Lemma 5.12 Let $G$ be a countable discrete group. Let $F$ be a nonempty finite subset of $G, 0<\tau \leq 1$, and $0<\eta<1 / 2$. Then for every large enough $d \in \mathbb{N}$, every map $\sigma: G \rightarrow \operatorname{Sym}(d)$ with sets $\mathcal{B}_{1}, \mathcal{B}_{2} \subseteq\{1, \ldots, d\}$ satisfying $\left|\mathcal{B}_{i}\right| \geq\left(\frac{\tau}{2}+\frac{2-2 \tau}{2-\tau}\right) d$ and

$$
\sigma_{s}(a) \neq \sigma_{t}(a)
$$

for all $a \in \mathcal{B}_{i}$ and distinct $s, t \in F$, and every $\mathcal{J}_{1}, \mathcal{J}_{2} \subseteq\{1, \ldots, d\}$ with $\left|\mathcal{J}_{i}\right| \geq \tau d$ for $i=1,2$, there exist $\mathcal{C}_{i} \subseteq \mathcal{B}_{i}$ such that

(1) for every $i=1,2$, the family $\left\{\sigma(F) c: c \in \mathcal{C}_{i}\right\}$ is $\eta$-disjoint and $\eta \frac{\tau}{16}$-covers $\{1, \ldots, d\}$, 
(2) there is a bijection $\varphi: \mathcal{C}_{1} \rightarrow \mathcal{C}_{2}$ such that for any $c \in \mathcal{C}_{1}$, one has $\mid\left\{s \in F: \sigma_{s}(c) \in\right.$ $\left.\mathcal{J}_{1}, \sigma_{s}(\varphi(c)) \in \mathcal{J}_{2}\right\}\left|\geq\left(\frac{\tau}{2}\right)^{2}\right| F \mid$.

Proof Note that for all distinct $a, c \in\{1, \ldots, d\}$ and $s \in F$ we have

$$
\sigma_{s}(a) \neq \sigma_{s}(c) \text {. }
$$

Taking $\lambda=\tau / 2$ and $\mathcal{J}=\mathcal{J}_{1}$ in Lemma 5.10, we find a $\mathcal{V}_{1} \subseteq \mathcal{B}_{1}$ such that $\left|\mathcal{V}_{1}\right| / d \geq$ $\frac{\left|\mathcal{B}_{1}\right|(1-\lambda) / d-1+\left|\mathcal{J}_{1}\right| / d}{1-\lambda} \geq \frac{(\tau / 2+(2-2 \tau) /(2-\tau))(1-\lambda)-1+\tau}{1-\lambda}=\frac{\tau}{2}$ and $\left|\sigma(F) a \cap \mathcal{J}_{1}\right| /|F| \geq \frac{\tau}{2}$ for all $a \in \mathcal{V}_{1}$. Observe that

$$
\sum_{c \in \mathcal{V}_{1}}|\sigma(F) c|=|F| \cdot\left|\mathcal{V}_{1}\right| \geq|F| \cdot \frac{\tau}{2} d=|F| \cdot\left(1-\frac{2-\tau}{2}\right) d,
$$

so that the family $\{\sigma(F) c\}_{c \in \mathcal{V}_{1}}$ is a $\frac{2-\tau}{2}$-even covering of $\{1, \ldots, d\}$ with multiplicity $|F|$. By Lemma 5.11, we can find a set $\mathcal{W}_{1} \subseteq \mathcal{V}_{1}$ such that the family $\{\sigma(F) c\}_{c \in \mathcal{W}_{1}}$ is $\eta$-disjoint and $\eta \frac{\tau}{2}$-covers $\{1, \ldots, d\}$. We may assume that $\left|\sigma(F) \mathcal{W}_{1}\right|<\eta \tau d / 2+|F|$.

List all the subsets of $F$ with cardinality $\lceil|F| \tau / 2\rceil$ as $F_{1}, \ldots, F_{n}$ for some $n \in \mathbb{N}$, where $\lceil x\rceil$ for a real number $x$ denotes the smallest integer no less than $x$. Then we can write $\mathcal{W}_{1}$ as the disjoint union of sets $\mathcal{W}_{1, j}$ for $1 \leq j \leq n$ such that $\sigma\left(F_{j}\right) c \subseteq \mathcal{J}_{1}$ for all $1 \leq j \leq n$ and $c \in \mathcal{W}_{1, j}$. Throwing away those empty $\mathcal{W}_{1, j}$, we may assume that each $\mathcal{W}_{1, j}$ is nonempty.

For each $1 \leq j \leq n$, taking $\lambda=\tau / 2$ and $\mathcal{J}=\mathcal{J}_{2}$ in Lemma 5.10, we find a $\mathcal{V}_{2, j} \subseteq$ $\mathcal{B}_{2}$ such that $\left|\mathcal{V}_{2, j}\right| / d \geq \frac{\left|\mathcal{B}_{2}\right|(1-\lambda) / d-1+\left|\mathcal{J}_{2}\right| / d}{1-\lambda} \geq \frac{(\tau / 2+(2-2 \tau) /(2-\tau))(1-\lambda)-1+\tau}{1-\lambda}=\frac{\tau}{2}$ and $\left|\sigma\left(F_{j}\right) a \cap \mathcal{J}_{2}\right| /\left|F_{j}\right| \geq \frac{\tau}{2}$ for all $a \in \mathcal{V}_{2, j}$.

We will recursively construct pairwise disjoint sets $\mathcal{C}_{2,1}, \ldots, \mathcal{C}_{2, n}$ such that the family $\left\{\sigma(F) c: c \in \mathcal{C}_{2, j}, 1 \leq j \leq n\right\}$ is $\eta$-disjoint, and $\mathcal{C}_{2, j} \subseteq \mathcal{V}_{2, j}$ and $\left|\mathcal{C}_{2, j}\right|=$ $\left\lfloor\left|\mathcal{W}_{1, j}\right| / 2\right\rfloor$ for every $1 \leq j \leq n$, where $\lfloor x\rfloor$ for any real number $x$ denotes the largest integer no bigger than $x$.

Note that

$$
\sum_{c \in \mathcal{V}_{2,1}}|\sigma(F) c|=|F| \cdot\left|\mathcal{V}_{2,1}\right| \geq|F| \cdot \frac{\tau}{2} d=|F| \cdot\left(1-\frac{2-\tau}{2}\right) d,
$$

so that the family $\{\sigma(F) c\}_{c \in \mathcal{V}_{2,1}}$ is a $\frac{2-\tau}{2}$-even covering of $\{1, \ldots, d\}$ with multiplicity $|F|$. By Lemma 5.11 , we can find a set $\mathcal{W}_{2,1} \subseteq \mathcal{V}_{2,1}$ such that the family $\{\sigma(F) c\}_{c \in \mathcal{W}_{2,1}}$ is $\eta$-disjoint and $\eta \frac{\tau}{2}$-covers $\{1, \ldots, d\}$. Note that

$$
\left|\mathcal{W}_{2,1}\right| \cdot|F| \geq\left|\sigma(F) \mathcal{W}_{2,1}\right| \geq \eta \frac{\tau}{2} d
$$

and since the family $\{\sigma(F) c\}_{c \in \mathcal{W}_{1}}$ is $\eta$-disjoint, we have

$$
\frac{1}{2}\left|\mathcal{W}_{1}\right| \cdot|F| \leq(1-\eta)\left|\mathcal{W}_{1}\right| \cdot|F| \leq\left|\sigma(F) \mathcal{W}_{1}\right|<\eta \frac{\tau}{2} d+|F|
$$

Thus

$$
\frac{1}{2}\left|\mathcal{W}_{1}\right| \cdot|F|<\left|\mathcal{W}_{2,1}\right| \cdot|F|+|F|
$$


and hence

$$
\left|\mathcal{W}_{2,1}\right|>\frac{1}{2}\left|\mathcal{W}_{1}\right|-1 \geq \frac{1}{2}\left|\mathcal{W}_{1,1}\right|-1 .
$$

Therefore, we can take a subset $\mathcal{C}_{2,1}$ of $\mathcal{W}_{2,1}$ with cardinality $\left\lfloor\frac{1}{2}\left|\mathcal{W}_{1,1}\right|\right\rfloor$.

Suppose that we have found pairwise disjoint sets $\mathcal{C}_{2,1}, \ldots, \mathcal{C}_{2, k}$ for some $1 \leq k<n$ such that the family $\left\{\sigma(F) c: c \in \mathcal{C}_{2, j}, 1 \leq j \leq k\right\}$ is $\eta$-disjoint, and $\mathcal{C}_{2, j} \subseteq \mathcal{V}_{2, j}$ and $\left|\mathcal{C}_{2, j}\right|=\left\lfloor\left|\mathcal{W}_{1, j}\right| / 2\right\rfloor$ for every $1 \leq j \leq k$. Note that

$$
\begin{aligned}
\sum_{c \in \mathcal{V}_{2, k+1} \cup \bigcup_{1 \leq j \leq k} \mathcal{C}_{2, j}}|\sigma(F) c| & =|F| \cdot\left|\mathcal{V}_{2, k+1} \cup \bigcup_{1 \leq j \leq k} \mathcal{C}_{2, j}\right| \\
& \geq|F| \cdot\left|\mathcal{V}_{2, k+1}\right| \geq|F| \cdot \frac{\tau}{2} d=|F| \cdot\left(1-\frac{2-\tau}{2}\right) d
\end{aligned}
$$

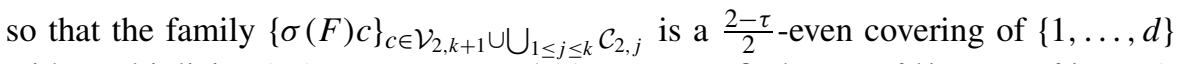
with multiplicity $|F|$. By Lemma 5.11 , we can find a set $\mathcal{W}_{2, k+1} \subseteq \mathcal{V}_{2, k+1} \backslash$

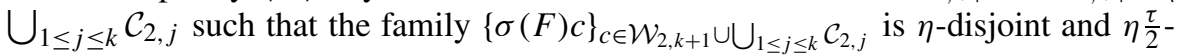
covers $\{1, \ldots, d\}$. Note that

$$
\left(\left|\mathcal{W}_{2, k+1}\right|+\sum_{1 \leq j \leq k}\left|\mathcal{C}_{2, j}\right|\right) \cdot|F| \geq\left|\sigma(F)\left(\mathcal{W}_{2, k+1} \cup \bigcup_{1 \leq j \leq k} \mathcal{C}_{2, j}\right)\right| \geq \eta \frac{\tau}{2} d .
$$

Thus, combining with (1), we have

$$
\begin{aligned}
\frac{1}{2} \sum_{1 \leq j \leq k+1}\left|\mathcal{W}_{1, j}\right| \cdot|F| & \leq \frac{1}{2}\left|\mathcal{W}_{1}\right| \cdot|F| \\
& <\left(\left|\mathcal{W}_{2, k+1}\right|+\sum_{1 \leq j \leq k}\left|\mathcal{C}_{2, j}\right|\right) \cdot|F|+|F| \\
& \leq\left(\left|\mathcal{W}_{2, k+1}\right|+\frac{1}{2} \sum_{1 \leq j \leq k}\left|\mathcal{W}_{1, j}\right|\right) \cdot|F|+|F|,
\end{aligned}
$$

and hence

$$
\left|\mathcal{W}_{2, k+1}\right|>\frac{1}{2}\left|\mathcal{W}_{1, k+1}\right|-1
$$

Therefore we can take a subset $\mathcal{C}_{2, k+1}$ of $\mathcal{W}_{2, k+1}$ with cardinality $\left\lfloor\frac{1}{2}\left|\mathcal{W}_{1, k+1}\right|\right\rfloor$. This completes the recursive construction.

For each $1 \leq j \leq n$ take a subset $\mathcal{C}_{1, j}$ of $\mathcal{W}_{1, j}$ with cardinality $\left\lfloor\left|\mathcal{W}_{1, j}\right| / 2\right\rfloor$. Set $\mathcal{C}_{i}=\bigcup_{1 \leq j \leq n} \mathcal{C}_{i, j}$ for $i=1,2$. Take a bijection $\varphi: \mathcal{C}_{1} \rightarrow \mathcal{C}_{2}$ such that $\varphi\left(\mathcal{C}_{1, j}\right)=\mathcal{C}_{2, j}$ for all $1 \leq \bar{j} \leq n$. For each $c \in \mathcal{C}_{1}$, considering $j$ such that $c \in \mathcal{C}_{1, j}$ one has

$$
\begin{aligned}
\left|\left\{s \in F: \sigma_{s}(c) \in \mathcal{J}_{1}, \sigma_{s}(\varphi(c)) \in \mathcal{J}_{2}\right\}\right| & \geq\left|\left\{s \in F_{j}: \sigma_{s}(\varphi(c)) \in \mathcal{J}_{2}\right\}\right| \\
& \geq \frac{\tau}{2}\left|F_{j}\right| \geq\left(\frac{\tau}{2}\right)^{2}|F| .
\end{aligned}
$$


Note that

$$
\left|\mathcal{W}_{1}\right| \cdot|F| \geq\left|\sigma(F) \mathcal{W}_{1}\right| \geq \eta \frac{\tau}{2} d
$$

and hence for $i=1,2$,

$$
\left|\mathcal{C}_{i}\right|=\sum_{1 \leq j \leq n}\left|\mathcal{C}_{i, j}\right| \geq \sum_{1 \leq j \leq n}\left(\frac{1}{2}\left|\mathcal{W}_{1, j}\right|-1\right) \geq \frac{1}{2}\left|\mathcal{W}_{1}\right|-2^{|F|} \geq \frac{1}{4}\left|\mathcal{W}_{1}\right|
$$

when $d$ is sufficiently large. Since the family $\left\{\sigma(F) c: c \in \mathcal{C}_{i}\right\}$ is $\eta$-disjoint, we get

$$
\left|\sigma(F) \mathcal{C}_{i}\right| \geq(1-\eta)\left|\mathcal{C}_{i}\right| \cdot|F| \geq(1-\eta) \eta \frac{\tau}{8} d \geq \eta \frac{\tau}{16} d
$$

Lemma 5.13 Let $G$ be a countable discrete group. Let $0<\tau \leq 1$, and $0<\eta<1 / 2$ with $\eta \frac{\tau}{16}<\frac{1-\tau^{\prime}}{24}$, where $\tau^{\prime}=\frac{\tau}{2}+\frac{2-2 \tau}{2-\tau}<1$. Then there are an $\ell \in \mathbb{N}$ and $\eta^{\prime}>0$ such that, whenever $e \in F_{1} \subseteq F_{2} \subseteq \cdots \subseteq F_{\ell}$ are finite subsets of $G$ with $\left|\left(F_{k-1}^{-1} F_{k}\right) \backslash F_{k}\right| \leq$ $\left|F_{k}\right|$ for $k=2, \ldots, \ell$, for every large enough $d \in \mathbb{N}$, every map $\sigma: G \rightarrow \operatorname{Sym}(d)$ with a set $\mathcal{B} \subseteq\{1, \ldots, d\}$ satisfying $|\mathcal{B}| \geq\left(1-\eta^{\prime}\right) d$ and

$$
\sigma_{s t}(a)=\sigma_{s} \sigma_{t}(a), \quad \sigma_{s}(a) \neq \sigma_{s^{\prime}}(a), \quad \sigma_{e}(a)=a
$$

for all $a \in \mathcal{B}$ and $s, t, s^{\prime} \in F_{\ell} \cup F_{\ell}^{-1}$ with $s \neq s^{\prime}$, and any $\mathcal{J}_{1}, \mathcal{J}_{2} \subseteq\{1, \ldots, d\}$ with $\left|\mathcal{J}_{i}\right| \geq \tau d$ for $i=1,2$, there exist $\mathcal{C}_{i, 1}, \ldots, \mathcal{C}_{i, \ell} \subseteq \mathcal{B}$ such that

(1) for every $i=1,2, k=1, \ldots, \ell$ and $c \in \mathcal{C}_{i, k}$, the map $s \mapsto \sigma_{s}(c)$ from $F_{k}$ to $\sigma\left(F_{k}\right)$ c is bijective,

(2) for every $i=1,2$, the sets $\sigma\left(F_{1}\right) \mathcal{C}_{i, 1}, \ldots, \sigma\left(F_{\ell}\right) \mathcal{C}_{i, \ell}$ are pairwise disjoint, the family $\bigcup_{k=1}^{\ell}\left\{\sigma\left(F_{k}\right) c: c \in \mathcal{C}_{i, k}\right\}$ is $\eta$-disjoint, and $(1-\eta) \frac{1-\tau^{\prime}}{24} d \leq$ $\left|\bigcup_{k=1}^{\ell} \sigma\left(F_{k}\right) \mathcal{C}_{i, k}\right| \leq\left(\frac{1}{1-\eta} \frac{1-\tau^{\prime}}{24}+\eta\right) d$,

(3) for every $k=1, \ldots, \ell$, there is a bijection $\varphi_{k}: \mathcal{C}_{1, k} \rightarrow \mathcal{C}_{2, k}$ such that for each $c \in \mathcal{C}_{1, k}$ one has $\left|\left\{s \in F_{k}: \sigma_{s}(c) \in \mathcal{J}_{1}, \sigma_{s}\left(\varphi_{k}(c)\right) \in \mathcal{J}_{2}\right\}\right| \geq\left(\frac{\tau}{2}\right)^{2}\left|F_{k}\right|$.

Proof Set $\eta^{\prime}=\frac{1-\tau^{\prime}}{2}$. Take $\ell$ to be the largest integer satisfying $\ell \eta \frac{\tau}{16} \leq \frac{1-\tau^{\prime}}{12}$. Then $\ell \eta \frac{\tau}{16} \geq \frac{1-\tau^{\prime}}{24}$. We will recursively construct sets $\mathcal{C}_{i, 1}^{\prime}, \ldots, \mathcal{C}_{i, \ell}^{\prime}$ in reverse order so that (i) for every $i=1,2$ and $1 \leq k \leq \ell$, the sets $\sigma\left(F_{k}\right) \mathcal{C}_{i, k}^{\prime}, \ldots, \sigma\left(F_{\ell}\right) \mathcal{C}_{i, \ell}^{\prime}$ are pairwise disjoint and the family $\bigcup_{n=k}^{\ell}\left\{\sigma\left(F_{n}\right) c: c \in \mathcal{C}_{i, n}^{\prime}\right\}$ is $\eta$-disjoint and $(\ell-k+1) \eta \frac{\tau}{16}$ covers $\{1, \ldots, d\}$, and (ii) for every $k=1, \ldots, \ell$, there is a bijection $\varphi_{k}: \mathcal{C}_{1, k}^{\prime} \rightarrow$ $\mathcal{C}_{2, k}^{\prime}$ such that for every $c \in \mathcal{C}_{1, k}^{\prime}$ one has $\left|\left\{s \in F_{k}: \sigma_{s}(c) \in \mathcal{J}_{1}, \sigma_{s}\left(\varphi_{k}(c)\right) \in \mathcal{J}_{2}\right\}\right| \geq$ $\left(\frac{\tau}{2}\right)^{2}\left|F_{k}\right|$.

Taking $\mathcal{B}_{i}=\mathcal{B}$ for $i=1,2$ in Lemma 5.12 we find $\mathcal{C}_{i, \ell}^{\prime} \subseteq \mathcal{B}$ for $i=1,2$ such that the family $\left\{\sigma\left(F_{\ell}\right) c: c \in \mathcal{C}_{i, \ell}^{\prime}\right\}$ is $\eta$-disjoint and $\eta \frac{\tau}{16}$-covers $\{1, \ldots, d\}$ for $i=1,2$ and there is a bijection $\varphi_{\ell}: \mathcal{C}_{1, \ell}^{\prime} \rightarrow \mathcal{C}_{2, \ell}^{\prime}$ with $\left|\left\{s \in F_{\ell}: \sigma_{s}(c) \in \mathcal{J}_{1}, \sigma_{s}\left(\varphi_{\ell}(c)\right) \in \mathcal{J}_{2}\right\}\right| \geq$ $\left(\frac{\tau}{2}\right)^{2}\left|F_{\ell}\right|$ for all $c \in \mathcal{C}_{1, \ell}^{\prime}$.

Suppose that $1 \leq k<\ell$ and we have found $\mathcal{C}_{i, k+1}^{\prime}, \ldots, \mathcal{C}_{i, \ell}^{\prime} \subseteq \mathcal{B}$ for $i=1,2$ such that the sets $\sigma\left(F_{k+1}\right) \mathcal{C}_{i, k+1}^{\prime}, \ldots, \sigma\left(F_{\ell}\right) \mathcal{C}_{i, \ell}^{\prime}$ are pairwise disjoint and the family $\bigcup_{n=k+1}^{\ell}\left\{\sigma\left(F_{n}\right) c: c \in \mathcal{C}_{i, n}^{\prime}\right\}$ is $\eta$-disjoint and $(\ell-k) \eta \frac{\tau}{16}$-covers $\{1, \ldots, d\}$ for 
each $i=1,2$, and there is a bijection $\varphi_{j}: \mathcal{C}_{1, j}^{\prime} \rightarrow \mathcal{C}_{2, j}^{\prime}$ with $\mid\left\{s \in F_{j}: \sigma_{s}(c) \in\right.$ $\left.\mathcal{J}_{1}, \sigma_{s}\left(\varphi_{j}(c)\right) \in \mathcal{J}_{2}\right\}\left|\geq\left(\frac{\tau}{2}\right)^{2}\right| F_{j} \mid$ for all $j=k+1, \ldots, \ell$ and $c \in \mathcal{C}_{1, j}^{\prime}$. Set $\theta_{i, k}=$ $\left|\bigcup_{j=k+1}^{\ell} \sigma\left(F_{j}\right) \mathcal{C}_{i, j}^{\prime}\right| / d$ and $\mathcal{B}_{i, k}=\left\{c \in \mathcal{B}: \sigma\left(F_{k}\right) c \cap\left(\bigcup_{j=k+1}^{\ell} \sigma\left(F_{j}\right) \mathcal{C}_{i, j}^{\prime}\right)=\emptyset\right\}$ for $i=1,2$.

If $1-\tau^{\prime}-\eta^{\prime}<3 \theta_{m, k}$ for some $m=1,2$, then we set $\mathcal{C}_{i, k}^{\prime}=\emptyset$ for each $i=1,2$. Then

$$
\sum_{j=k}^{\ell}\left|\mathcal{C}_{m, j}^{\prime}\right| \cdot\left|F_{j}\right| \geq\left|\bigcup_{j=k}^{\ell} \sigma\left(F_{j}\right) \mathcal{C}_{m, j}^{\prime}\right|=\theta_{m, k} d \geq \frac{1-\tau^{\prime}-\eta^{\prime}}{3} d=\frac{1-\tau^{\prime}}{6} d .
$$

Since the family $\bigcup_{j=k}^{\ell}\left\{\sigma\left(F_{j}\right) c: c \in \mathcal{C}_{i, j}^{\prime}\right\}$ is $\eta$-disjoint, one has

$$
\begin{aligned}
\left|\bigcup_{j=k}^{\ell} \sigma\left(F_{j}\right) \mathcal{C}_{i, j}^{\prime}\right| & \geq(1-\eta) \sum_{j=k}^{\ell}\left|\mathcal{C}_{i, j}^{\prime}\right| \cdot\left|F_{j}\right| \geq(1-\eta) \frac{1-\tau^{\prime}}{6} d \\
& \geq \frac{1-\tau^{\prime}}{12} d \geq \ell \eta \frac{\tau}{16} d \geq(\ell-k+1) \eta \frac{\tau}{16} d
\end{aligned}
$$

for $i=1,2$.

Assume that $1-\tau^{\prime}-\eta^{\prime} \geq 3 \theta_{i, k}$ for every $i=1,2$. Let $i \in\{1,2\}$. For every $c \in$ $\mathcal{B} \backslash \mathcal{B}_{i, k}$ we have $\sigma_{s}(c)=\sigma_{t}(a)$ for some $j \in\{k+1, \ldots, \ell\}, a \in \mathcal{C}_{i, j}^{\prime}, t \in F_{j}$, and $s \in F_{k}$, and hence

$$
c=\sigma_{s^{-1}} \sigma_{s}(c)=\sigma_{s^{-1}} \sigma_{t}(a)=\sigma_{s^{-1} t}(a) \in \bigcup_{j=k+1}^{\ell} \sigma\left(F_{k}^{-1} F_{j}\right) \mathcal{C}_{i, j}^{\prime}
$$

Therefore

$$
\mathcal{B} \backslash \mathcal{B}_{i, k} \subseteq \bigcup_{j=k+1}^{\ell} \sigma\left(F_{k}^{-1} F_{j}\right) \mathcal{C}_{i, j}^{\prime}
$$

Since the family $\bigcup_{j=k+1}^{\ell}\left\{\sigma\left(F_{j}\right) c: c \in \mathcal{C}_{i, j}^{\prime}\right\}$ is $\eta$-disjoint we have

$$
\frac{1}{2} \sum_{j=k+1}^{\ell}\left|F_{j}\right| \cdot\left|\mathcal{C}_{i, j}^{\prime}\right| \leq \sum_{j=k+1}^{\ell}(1-\eta)\left|F_{j}\right| \cdot\left|\mathcal{C}_{i, j}^{\prime}\right| \leq\left|\bigcup_{j=k+1}^{\ell} \sigma\left(F_{j}\right) \mathcal{C}_{i, j}^{\prime}\right|=\theta_{i, k} d .
$$

Thus

$$
\begin{aligned}
\left|\bigcup_{j=k+1}^{\ell} \sigma\left(F_{k}^{-1} F_{j}\right) \mathcal{C}_{i, j}^{\prime}\right| & \leq\left|\bigcup_{j=k+1}^{\ell} \sigma\left(\left(F_{k}^{-1} F_{j}\right) \backslash F_{j}\right) \mathcal{C}_{i, j}^{\prime}\right|+\left|\bigcup_{j=k+1}^{\ell} \sigma\left(F_{j}\right) \mathcal{C}_{i, j}^{\prime}\right| \\
& \leq \sum_{j=k+1}^{\ell}\left|\left(F_{k}^{-1} F_{j}\right) \backslash F_{j}\right| \cdot\left|\mathcal{C}_{i, j}^{\prime}\right|+\theta_{i, k} d
\end{aligned}
$$




$$
\begin{aligned}
& \leq \sum_{j=k+1}^{\ell}\left|\left(F_{j-1}^{-1} F_{j}\right) \backslash F_{j}\right| \cdot\left|\mathcal{C}_{i, j}^{\prime}\right|+\theta_{i, k} d \\
& \leq \sum_{j=k+1}^{\ell}\left|F_{j}\right| \cdot\left|\mathcal{C}_{i, j}^{\prime}\right|+\theta_{i, k} d \\
& \leq 3 \theta_{i, k} d .
\end{aligned}
$$

Therefore

$$
\begin{aligned}
\left|\mathcal{B}_{i, k}\right|=|\mathcal{B}|-\left|\mathcal{B} \backslash \mathcal{B}_{i, k}\right| & \geq\left(1-\eta^{\prime}\right) d-\left|\bigcup_{j=k+1}^{\ell} \sigma\left(F_{k}^{-1} F_{j}\right) \mathcal{C}_{i, j}^{\prime}\right| \\
& \geq\left(1-\eta^{\prime}\right) d-3 \theta_{i, k} d \\
& \geq \tau^{\prime} d .
\end{aligned}
$$

Taking $\mathcal{B}_{i}=\mathcal{B}_{i, k}$ in Lemma 5.12, we find $\mathcal{C}_{i, k}^{\prime} \subseteq \mathcal{B}_{i, k}$ for $i=1,2$ such that the family $\left\{\sigma\left(F_{k}\right) c: c \in \mathcal{C}_{i, k}^{\prime}\right\}$ is $\eta$-disjoint and $\eta \frac{\tau}{16}$-covers $\{1, \ldots, d\}$ for $i=1,2$ and there is a bijection $\varphi_{k}: \mathcal{C}_{1, k}^{\prime} \rightarrow \mathcal{C}_{2, k}^{\prime}$ with $\left|\left\{s \in F_{k}: \sigma_{s}(c) \in \mathcal{J}_{1}, \sigma_{s}\left(\varphi_{k}(c)\right) \in \mathcal{J}_{2}\right\}\right| \geq$ $\left(\frac{\tau}{2}\right)^{2}\left|F_{k}\right|$ for all $c \in \mathcal{C}_{1, k}^{\prime}$. Then for each $i=1,2$, the sets $\sigma\left(F_{k}\right) \mathcal{C}_{i, k}^{\prime}, \ldots, \sigma\left(F_{\ell}\right) \mathcal{C}_{i, \ell}^{\prime}$ are pairwise disjoint, the family $\bigcup_{j=k}^{\ell}\left\{\sigma\left(F_{j}\right) c: c \in \mathcal{C}_{i, j}^{\prime}\right\}$ is $\eta$-disjoint, and

$$
\begin{aligned}
\left|\bigcup_{j=k}^{\ell} \sigma\left(F_{j}\right) \mathcal{C}_{i, j}^{\prime}\right| & =\left|\sigma\left(F_{k}\right) \mathcal{C}_{i, k}^{\prime}\right|+\left|\bigcup_{j=k+1}^{\ell} \sigma\left(F_{j}\right) \mathcal{C}_{i, j}^{\prime}\right| \\
& \geq \eta \frac{\tau}{16} d+(\ell-k) \eta \frac{\tau}{16} d=(\ell-k+1) \eta \frac{\tau}{16} d,
\end{aligned}
$$

completing the recursive construction.

When $d$ is large enough, take a subset $\mathcal{C}_{1, k}$ of $\mathcal{C}_{1, k}^{\prime}$ for each $1 \leq k \leq \ell$ such that

$$
\frac{1-\tau^{\prime}}{24} d \leq\left|\bigcup_{k=1}^{\ell} \sigma\left(F_{k}\right) \mathcal{C}_{1, k}\right| \leq \frac{1-\tau^{\prime}}{24} d+\left|F_{\ell}\right| \leq \frac{1-\tau^{\prime}}{24} d+\eta(1-\eta) d .
$$

Set $\mathcal{C}_{2, k}=\varphi_{k}\left(\mathcal{C}_{1, k}\right)$ for each $1 \leq k \leq \ell$. Since the families $\bigcup_{k=1}^{\ell}\left\{\sigma\left(F_{k}\right) c: c \in \mathcal{C}_{i, k}\right\}$ for $i=1,2$ are $\eta$-disjoint, we have

$$
\left|\bigcup_{k=1}^{\ell} \sigma\left(F_{k}\right) \mathcal{C}_{2, k}\right| \geq(1-\eta)\left|\bigcup_{k=1}^{\ell} \sigma\left(F_{k}\right) \mathcal{C}_{1, k}\right| \geq(1-\eta) \frac{1-\tau^{\prime}}{24} d
$$

and

$$
\left|\bigcup_{k=1}^{\ell} \sigma\left(F_{k}\right) \mathcal{C}_{2, k}\right| \leq \frac{1}{1-\eta}\left|\bigcup_{k=1}^{\ell} \sigma\left(F_{k}\right) \mathcal{C}_{1, k}\right| \leq\left(\frac{1}{1-\eta} \cdot \frac{1-\tau^{\prime}}{24}+\eta\right) d .
$$


We are ready to prove Theorem 5.8.

Proof of Theorem 5.8 It suffices to show that for any internal sets $Y=\prod_{\mathfrak{F}} \mathcal{Y}_{n}$ and $Z=\prod_{\mathfrak{F}} \mathcal{Z}_{n}$ with strictly positive measure, there is some $s \in G^{\prime}$ with $\mu(Z \cap s Y)>0$. In turn it is enough to show that there is some $\lambda>0$ such that for every finite subset $F$ of $G$ and $\varepsilon>0$ the set of all $n \in \mathbb{N}$ for which there is some $\varphi \in \operatorname{Sym}\left(d_{n}\right)$ satisfying $\rho_{\text {Hamm }}\left(\varphi \sigma_{s}, \sigma_{s} \varphi\right)<\varepsilon$ for all $s \in F$ and $\frac{\left|\varphi\left(\mathcal{Y}_{n}\right) \cap \mathcal{Z}_{n}\right|}{d_{n}} \geq \lambda$ belongs to $\mathfrak{F}$.

Set $\tau=\min (\mu(Y), \mu(Z)) / 2, \tau^{\prime}=\frac{\tau}{2}+\frac{2-2 \tau}{2-\tau}$, and $\lambda=\frac{\tau^{2}\left(1-\tau^{\prime}\right)}{384}$. Take $0<\eta<1 / 2$ to be a small number with $\eta \frac{\tau}{16}<\frac{1-\tau^{\prime}}{24}$, to be determined in a moment. Then the set $V$ of all $n \in \mathbb{N}$ satisfying $\min \left(\left|\mathcal{Y}_{n}\right| / d_{n},\left|\mathcal{Z}_{n}\right| / d_{n}\right) \geq \tau$ belongs to $\mathfrak{F}$. Let $\ell$ and $\eta^{\prime}$ be as in Lemma 5.13. We may assume that $\eta^{\prime}<\varepsilon / 2$. Set $\theta=\frac{1}{(1-\eta)^{2}} \frac{1-\tau^{\prime}}{24}+\frac{\eta}{1-\eta}+\eta^{\prime}$. Let $\ell^{\prime}, \kappa$, and $\eta^{\prime \prime}$ be as in Lemma 5.9. Take $F_{1}^{\prime} \subseteq F_{2}^{\prime} \subseteq \cdots \subseteq F_{\ell^{\prime}}^{\prime} \subseteq F_{1} \subseteq F_{2} \subseteq \cdots \subseteq F_{\ell}$ to be finite subsets of $G$ containing $e$ such that

(1) $\left|\left(\left(F_{\ell^{\prime}}^{\prime}\right)^{-1} F_{k}\right) \backslash F_{k}\right|<\eta\left|F_{k}\right|$ for all $k=1, \ldots, \ell$,

(2) $\left|\left(\left(F_{k-1}^{\prime}\right)^{-1} F_{k}^{\prime}\right) \backslash F_{k}^{\prime}\right|<\kappa\left|F_{k}^{\prime}\right|$ for all $k=2, \ldots, \ell^{\prime}$ and $\left|\tilde{F}_{k}^{\prime}\right| \geq(1-\eta)\left|F_{k}^{\prime}\right|$ for all $k=1, \ldots, \ell^{\prime}$, where $\tilde{F}_{k}^{\prime}=\left\{s \in F_{k}^{\prime}: F s \subseteq F_{k}^{\prime}\right\}$, and

(3) $\left|\left(F_{k-1}^{-1} F_{k}\right) \backslash F_{k}\right|<\left|F_{k}\right|$ for all $k=2, \ldots, \ell$ and $\left|\tilde{F}_{k}\right| \geq(1-\eta)\left|F_{k}\right|$ for all $k=$ $1, \ldots, \ell$, where $\tilde{F}_{k}=\left\{s \in F_{k}: F s \subseteq F_{k}\right\}$.

Then we have $\lambda_{1}, \ldots, \lambda_{\ell^{\prime}}$ as in Lemma 5.9. When $n \in V$ is large enough, one has $|\mathcal{B}| \geq\left(1-\min \left(\eta^{\prime}, \eta^{\prime \prime}\right)\right) d_{n}$ where $\mathcal{B}$ denotes the set of all $a \in\left\{1, \ldots, d_{n}\right\}$ satisfying

$$
\sigma_{n, s t}(a)=\sigma_{n, s} \sigma_{n, t}(a), \quad \sigma_{n, s}(a) \neq \sigma_{n, s^{\prime}}(a), \quad \sigma_{n, e}(a)=a
$$

for all $s, t \in\left(F \cup F_{\ell}\right) \cup\left(F \cup F_{\ell}\right)^{-1}$ and distinct $s, s^{\prime} \in F_{\ell} \cup F_{\ell}^{-1}$, and one has $\mathcal{C}_{i, 1}, \ldots, \mathcal{C}_{i, \ell} \subseteq \mathcal{B}$ for $i=1,2$ as in Lemma 5.13 for $d=d_{n}, \sigma=\sigma_{n}, \mathcal{J}_{1}=\mathcal{Y}_{n}$, and $\mathcal{J}_{2}=\mathcal{Z}_{n}$.

Let $i \in\{1,2\}$. Set $\mathcal{V}_{i}=\left\{c \in \mathcal{B}: \sigma_{n}\left(F_{\ell^{\prime}}^{\prime}\right) c \cap \bigcup_{k=1}^{\ell} \sigma_{n}\left(F_{k}\right) \mathcal{C}_{i, k}=\emptyset\right\}$. Then $\mathcal{B} \backslash \mathcal{V}_{i} \subseteq$ $\bigcup_{k=1}^{\ell} \sigma_{n}\left(\left(F_{\ell^{\prime}}^{\prime}\right)^{-1} F_{k}\right) \mathcal{C}_{i, k}$. Since the family $\bigcup_{k=1}^{\ell}\left\{\sigma_{n}\left(F_{k}\right) c: c \in \mathcal{C}_{i, k}\right\}$ is $\eta$-disjoint, one has

$$
\begin{aligned}
\left|\mathcal{B} \backslash \mathcal{V}_{i}\right| & \leq\left|\bigcup_{k=1}^{\ell} \sigma_{n}\left(\left(F_{\ell^{\prime}}^{\prime}\right)^{-1} F_{k}\right) \mathcal{C}_{i, k}\right| \\
& \leq\left|\bigcup_{k=1}^{\ell} \sigma_{n}\left(\left(\left(F_{\ell^{\prime}}^{\prime}\right)^{-1} F_{k}\right) \backslash F_{k}\right) \mathcal{C}_{i, k}\right|+\left|\bigcup_{k=1}^{\ell} \sigma_{n}\left(F_{k}\right) \mathcal{C}_{i, k}\right| \\
& \leq \sum_{k=1}^{\ell}\left|\sigma_{n}\left(\left(\left(F_{\ell^{\prime}}^{\prime}\right)^{-1} F_{k}\right) \backslash F_{k}\right) \mathcal{C}_{i, k}\right|+\left|\bigcup_{k=1}^{\ell} \sigma_{n}\left(F_{k}\right) \mathcal{C}_{i, k}\right| \\
& \leq \sum_{k=1}^{\ell}\left|\left(\left(F_{\ell^{\prime}}^{\prime}\right)^{-1} F_{k}\right) \backslash F_{k}\right| \cdot\left|\mathcal{C}_{i, k}\right|+\left|\bigcup_{k=1}^{\ell} \sigma_{n}\left(F_{k}\right) \mathcal{C}_{i, k}\right|
\end{aligned}
$$




$$
\begin{aligned}
& \leq \eta \sum_{k=1}^{\ell}\left|F_{k}\right| \cdot\left|\mathcal{C}_{i, k}\right|+\left|\bigcup_{k=1}^{\ell} \sigma_{n}\left(F_{k}\right) \mathcal{C}_{i, k}\right| \\
& \leq\left(\frac{\eta}{1-\eta}+1\right)\left|\bigcup_{k=1}^{\ell} \sigma_{n}\left(F_{k}\right) \mathcal{C}_{i, k}\right| \\
& \leq \frac{1}{(1-\eta)^{2}} \frac{1-\tau^{\prime}}{24} d_{n}+\frac{\eta}{1-\eta} d_{n},
\end{aligned}
$$

and thus

$$
\left|\mathcal{V}_{i}\right|=|\mathcal{B}|-\left|\mathcal{B} \backslash \mathcal{V}_{i}\right| \geq(1-\theta) d_{n}
$$

Take $\delta>0$ with $2 \eta \delta+2 \eta \delta \ell^{\prime}+2 \delta \ell^{\prime} \leq \eta$. Taking $\mathcal{V}=\mathcal{V}_{i}$ in Lemma 5.9, when $n \in V$ is large enough, we find $\mathcal{C}_{i, 1}^{\prime}, \ldots, \mathcal{C}_{i, \ell^{\prime}}^{\prime} \subseteq \mathcal{V}_{i}$ such that

(1) for every $k=1, \ldots, \ell^{\prime}$ and $c \in \mathcal{C}_{i, k}^{\prime}$, the map $s \mapsto \sigma_{n, s}(c)$ from $F_{k}^{\prime}$ to $\sigma_{n}\left(F_{k}^{\prime}\right) c$ is bijective,

(2) the sets $\sigma_{n}\left(F_{1}^{\prime}\right) \mathcal{C}_{i, 1}^{\prime}, \ldots, \sigma_{n}\left(F_{\ell^{\prime}}^{\prime}\right) \mathcal{C}_{i, \ell^{\prime}}^{\prime}$ are pairwise disjoint and the family $\bigcup_{k=1}^{\ell^{\prime}}\left\{\sigma_{n}\left(F_{k}^{\prime}\right) c: c \in \mathcal{C}_{i, k}^{\prime}\right\}$ is $\eta$-disjoint and $(1-\theta-\eta)$-covers $\{1, \ldots, d\}$,

(3) $\sum_{k=1}^{\ell^{\prime}}|| \sigma_{n}\left(F_{k}^{\prime}\right) \mathcal{C}_{i, k}^{\prime}\left|/ d_{n}-\lambda_{k}\right|<\delta$.

Note that

$$
\sum_{k=1}^{\ell^{\prime}} \lambda_{k} \leq \frac{1}{d_{n}}\left|\bigcup_{k=1} \sigma_{n}\left(F_{k}^{\prime}\right) \mathcal{C}_{1, k}^{\prime}\right|+\delta \leq 1+\delta .
$$

For each $1 \leq k \leq \ell^{\prime}$, take $\mathcal{C}_{1, k}^{\prime \prime} \subseteq \mathcal{C}_{1, k}^{\prime}$ and $\mathcal{C}_{2, k}^{\prime \prime} \subseteq \mathcal{C}_{2, k}^{\prime}$ with

$$
\left|\mathcal{C}_{1, k}^{\prime \prime}\right|=\left|\mathcal{C}_{2, k}^{\prime \prime}\right|=\min \left(\left|\mathcal{C}_{1, k}^{\prime}\right|,\left|\mathcal{C}_{2, k}^{\prime}\right|\right)
$$

Take a bijection $\varphi_{k}^{\prime}: \mathcal{C}_{1, k}^{\prime \prime} \rightarrow \mathcal{C}_{2, k}^{\prime \prime}$. We have

$$
\begin{aligned}
\left|\mathcal{C}_{1, k}^{\prime}\right| \cdot\left|F_{k}^{\prime}\right|-\left|\mathcal{C}_{2, k}^{\prime}\right| \cdot\left|F_{k}^{\prime}\right| & \leq \frac{1}{1-\eta}\left|\sigma_{n}\left(F_{k}^{\prime}\right) \mathcal{C}_{1, k}^{\prime}\right|-\left|\sigma_{n}\left(F_{k}^{\prime}\right) \mathcal{C}_{2, k}^{\prime}\right| \\
& \leq(1+2 \eta)\left|\sigma_{n}\left(F_{k}^{\prime}\right) \mathcal{C}_{1, k}^{\prime}\right|-\left|\sigma_{n}\left(F_{k}^{\prime}\right) \mathcal{C}_{2, k}^{\prime}\right| \\
& \leq(1+2 \eta)\left(\lambda_{k}+\delta\right) d_{n}-\left(\lambda_{k}-\delta\right) d_{n} \\
& =\left(2 \eta \lambda_{k}+2 \eta \delta+2 \delta\right) d_{n},
\end{aligned}
$$

and similarly $\left|\mathcal{C}_{2, k}^{\prime}\right| \cdot\left|F_{k}^{\prime}\right|-\left|\mathcal{C}_{1, k}^{\prime}\right| \cdot\left|F_{k}^{\prime}\right| \leq\left(2 \eta \lambda_{k}+2 \eta \delta+2 \delta\right) d_{n}$. Thus for each $i=1,2$ one has

$$
\begin{aligned}
\left|\sigma_{n}\left(F_{k}^{\prime}\right)\left(\mathcal{C}_{i, k}^{\prime} \backslash \mathcal{C}_{i, k}^{\prime \prime}\right)\right| & \leq\left|\mathcal{C}_{i, k}^{\prime} \backslash \mathcal{C}_{i, k}^{\prime \prime}\right| \cdot\left|F_{k}^{\prime}\right| \\
& =|| \mathcal{C}_{1, k}^{\prime}|\cdot| F_{k}^{\prime}|-| \mathcal{C}_{2, k}^{\prime}|\cdot| F_{k}^{\prime}|| \leq\left(2 \eta \lambda_{k}+2 \eta \delta+2 \delta\right) d_{n},
\end{aligned}
$$


and hence

$$
\begin{aligned}
\left|\bigcup_{k=1}^{\ell^{\prime}} \sigma_{n}\left(F_{k}^{\prime}\right)\left(\mathcal{C}_{i, k}^{\prime} \backslash \mathcal{C}_{i, k}^{\prime \prime}\right)\right| & =\sum_{k=1}^{\ell^{\prime}}\left|\sigma_{n}\left(F_{k}^{\prime}\right)\left(\mathcal{C}_{i, k}^{\prime} \backslash \mathcal{C}_{i, k}^{\prime \prime}\right)\right| \\
& \leq \sum_{k=1}^{\ell^{\prime}}\left(2 \eta \lambda_{k}+2 \eta \delta+2 \delta\right) d_{n} \\
& \leq\left(2 \eta(1+\delta)+2 \eta \delta \ell^{\prime}+2 \delta \ell^{\prime}\right) d_{n} \leq 3 \eta d_{n} .
\end{aligned}
$$

Therefore,

$$
\begin{aligned}
\left|\bigcup_{k=1}^{\ell^{\prime}} \sigma_{n}\left(F_{k}^{\prime}\right) \mathcal{C}_{i, k}^{\prime \prime}\right| & \geq\left|\bigcup_{k=1}^{\ell^{\prime}} \sigma_{n}\left(F_{k}^{\prime}\right) \mathcal{C}_{i, k}^{\prime}\right|-\left|\bigcup_{k=1}^{\ell^{\prime}} \sigma_{n}\left(F_{k}^{\prime}\right)\left(\mathcal{C}_{i, k}^{\prime} \backslash \mathcal{C}_{i, k}^{\prime \prime}\right)\right| \\
& \geq(1-\theta-\eta) d_{n}-3 \eta d_{n}=(1-\theta-4 \eta) d_{n}
\end{aligned}
$$

for $i=1,2$.

Since the families $\bigcup_{k=1}^{\ell}\left\{\sigma_{n}\left(F_{k}\right) c: c \in \mathcal{C}_{i, k}\right\}$ for $i=1,2$ are $\eta$-disjoint, we can find $F_{i, c} \subseteq F_{k}$ with $\left|F_{i, c}\right| \geq(1-\eta)\left|F_{k}\right|$ for all $i=1,2, k=1, \ldots, \ell$, and $c \in \mathcal{C}_{i, k}$ so that for each $i=1,2$, the sets $\sigma_{n}\left(F_{i, c}\right) c$ for $c \in \bigcup_{k=1}^{\ell} \mathcal{C}_{i, k}$ are pairwise disjoint. For every $k=1, \ldots, \ell$ and $c \in \mathcal{C}_{1, k}$, set $\bar{F}_{c}=F_{1, c} \cap F_{2, \varphi_{k}(c)}$ and $\hat{F}_{c}=\left\{s \in \bar{F}_{c}: F s \subseteq \bar{F}_{c}\right\}$. Then $\left|\bar{F}_{c}\right| \geq(1-2 \eta)\left|F_{k}\right|$ and $\left|\hat{F}_{c}\right| \geq\left|\tilde{F}_{k}\right|-2 \eta|F| \cdot\left|F_{k}\right| \geq(1-(2|F|+1) \eta)\left|F_{k}\right|$.

Similarly, for every $1 \leq k \leq \ell^{\prime}$ and $c \in \mathcal{C}_{1, k}^{\prime \prime}$, we find some $\tilde{F}_{c}^{\prime} \subseteq F_{k}^{\prime}$ with $\left|\tilde{F}_{c}^{\prime}\right| \geq$ $(1-2 \eta)\left|F_{k}^{\prime}\right|$ such that the sets $\sigma_{n}\left(\tilde{F}_{c}^{\prime}\right) c$ for $c \in \bigcup_{k=1}^{\ell} \mathcal{C}_{1, k}^{\prime \prime}$, as well as the sets $\sigma_{n}\left(\tilde{F}_{c}^{\prime}\right) \varphi_{k}^{\prime}(c)$ for $c \in \bigcup_{k=1}^{\ell} \mathcal{C}_{1, k}^{\prime \prime}$, are pairwise disjoint. Setting $\hat{F}_{c}^{\prime}=\left\{s \in \bar{F}_{c}^{\prime}: F s \subseteq\right.$ $\left.\bar{F}_{c}^{\prime}\right\}$, we have $\left|\hat{F}_{c}^{\prime}\right| \geq(1-(2|F|+1) \eta)\left|F_{k}^{\prime}\right|$.

Note that

$$
\begin{aligned}
\left|\bigcup_{k=1}^{\ell} \bigcup_{c \in \mathcal{C}_{1, k}} \sigma_{n}\left(\hat{F}_{c}\right) c\right| & =\sum_{k=1}^{\ell} \sum_{c \in \mathcal{C}_{1, k}}\left|\hat{F}_{c}\right| \\
& \geq \sum_{k=1}^{\ell} \sum_{c \in \mathcal{C}_{1, k}}(1-(2|F|+1) \eta)\left|F_{k}\right| \\
& \geq(1-(2|F|+1) \eta)\left|\bigcup_{k=1}^{\ell} \sigma_{n}\left(F_{k}\right) \mathcal{C}_{1, k}\right| \\
& \geq(1-(2|F|+1) \eta)(1-\eta) \frac{1-\tau^{\prime}}{24} d_{n} .
\end{aligned}
$$

Similarly,

$$
\begin{aligned}
\left|\bigcup_{k=1}^{\ell^{\prime}} \bigcup_{c \in \mathcal{C}_{1, k}^{\prime \prime}} \sigma_{n}\left(\hat{F}_{c}^{\prime}\right) c\right| & \geq(1-(2|F|+1) \eta)\left|\bigcup_{k=1}^{\ell^{\prime}} \sigma_{n}\left(F_{k}^{\prime}\right) \mathcal{C}_{1, k}^{\prime \prime}\right| \\
& \geq(1-(2|F|+1) \eta)(1-\theta-4 \eta) d_{n}
\end{aligned}
$$


Set $\mathcal{W}=\left(\bigcup_{k=1}^{\ell} \bigcup_{c \in \mathcal{C}_{1, k}} \sigma_{n}\left(\hat{F}_{c}\right) c\right) \cup\left(\bigcup_{k=1}^{\ell^{\prime}} \bigcup_{c \in \mathcal{C}_{1, k}^{\prime \prime}} \sigma_{n}\left(\hat{F}_{c}^{\prime}\right) c\right)$. Then

$$
|\mathcal{W}| \geq(1-(2|F|+1) \eta)(1-\eta) \frac{1-\tau^{\prime}}{24} d_{n}+(1-(2|F|+1) \eta)(1-\theta-4 \eta) d_{n}
$$

Take a $\varphi \in \operatorname{Sym}\left(d_{n}\right)$ such that $\varphi\left(\sigma_{n, s}(c)\right)=\sigma_{n, s}\left(\varphi_{k}(c)\right)$ for all $k=1, \ldots, \ell, c \in$ $\mathcal{C}_{1, k}$, and $s \in \bar{F}_{c}$, and $\varphi\left(\sigma_{n, s}(c)\right)=\sigma_{n, s}\left(\varphi_{k}^{\prime}(c)\right)$ for all $k=1, \ldots, \ell^{\prime}, c \in \mathcal{C}_{1, k}^{\prime \prime}$, and $s \in \bar{F}_{c}^{\prime}$. For every $s \in F$, note that $\sigma_{n, s} \varphi=\varphi \sigma_{n, s}$ on $\mathcal{W}$, and hence

$$
\begin{aligned}
\rho_{\mathrm{Hamm}}\left(\sigma_{n, s} \varphi, \varphi \sigma_{n, s}\right) \leq & 1-\frac{|\mathcal{W}|}{d_{n}} \leq 1-(1-(2|F|+1) \eta)(1-\eta) \frac{1-\tau^{\prime}}{24} \\
& -(1-(2|F|+1) \eta)(1-\theta-4 \eta)<\varepsilon
\end{aligned}
$$

when $\eta$ is small enough. We also have

$$
\begin{aligned}
\left|\varphi\left(\mathcal{Y}_{n}\right) \cap \mathcal{Z}_{n}\right| & \geq \sum_{k=1}^{\ell} \sum_{c \in \mathcal{C}_{1, k}}\left|\left\{s \in \bar{F}_{c}: \sigma_{n, s}(c) \in \mathcal{Y}_{n}, \sigma_{n, s}\left(\varphi_{k}(c)\right) \in \mathcal{Z}_{n}\right\}\right| \\
& \geq \sum_{k=1}^{\ell} \sum_{c \in \mathcal{C}_{1, k}}\left(\left|\left\{s \in F_{k}: \sigma_{n, s}(c) \in \mathcal{Y}_{n}, \sigma_{n, s}\left(\varphi_{k}(c)\right) \in \mathcal{Z}_{n}\right\}\right|-2 \eta\left|F_{k}\right|\right) \\
& \geq \sum_{k=1}^{\ell} \sum_{c \in \mathcal{C}_{1, k}}\left(\left(\frac{\tau}{2}\right)^{2}\left|F_{k}\right|-2 \eta\left|F_{k}\right|\right) \\
& \geq \sum_{k=1}^{\ell} \sum_{c \in \mathcal{C}_{1, k}} \frac{\tau^{2}}{8}\left|F_{k}\right| \geq \frac{\tau^{2}}{8}\left|\bigcup_{k=1}^{\ell} \sigma_{n}\left(F_{k}\right) \mathcal{C}_{1, k}\right| \\
& \geq \frac{\tau^{2}}{8}(1-\eta) \frac{1-\tau^{\prime}}{24} d_{n} \geq \frac{\tau^{2}}{16} \frac{1-\tau^{\prime}}{24} d_{n}=\lambda d_{n}
\end{aligned}
$$

when $\eta$ is small enough.

Question 5.14 Does every countable sofic group $G$ admit a sofic approximation sequence $\Sigma$ such that the action of $G^{\prime}$ on $\left(\prod_{\mathfrak{F}}\left\{1, \ldots, d_{i}\right\}, \mathfrak{B}, \mu\right)$ is ergodic?

\section{IE-tuples and Algebraic Actions}

By an algebraic action we mean an action of a countable discrete group $G$ on a compact metrizable Abelian group $X$ by (continuous) automorphisms. The structure of such an action is captured by the Pontryagin dual $\widehat{X}$ viewed as a module over the integral group ring $\mathbb{Z} G$. The ring $\mathbb{Z} G$ consists of the finitely supported $\mathbb{Z}$-valued functions on $G$, which we write in the form $\sum_{s \in G} f_{s} s$, with addition $\left(\sum_{s \in G} f_{s} s\right)+\left(\sum_{s \in G} g_{s} s\right)=\sum_{s \in G}\left(f_{s}+g_{s}\right) s$ and multiplication $\left(\sum_{s \in G} f_{s} s\right)\left(\sum_{s \in G} g_{s} s\right)=\sum_{s \in G}\left(\sum_{t \in G} f_{t} g_{t^{-1}}\right) s$. 
Given a matrix $A$ in $M_{n}(\mathbb{Z} G)$, the left action of $G$ on $(\mathbb{Z} G)^{n} /(\mathbb{Z} G)^{n} A$ gives rise via Pontryagin duality to the algebraic action $\left.G \curvearrowright X_{A}:=(\mathbb{Z} G)^{n /(\mathbb{Z}} G\right)^{n} A$. Write $A^{*}$ for the matrix in $M_{n}(\mathbb{Z} G)$ whose $(i, j)$ entry is the result of applying the involution $\left(\sum_{s \in G} f_{s} s\right)^{*}=\sum_{s \in G} f_{s} s^{-1}$ to the $(j, i)$ entry of $A$. Viewing $\overline{(\mathbb{Z G})^{n}}$ as $\left((\mathbb{R} / \mathbb{Z})^{G}\right)^{n}$, we can then identify $X_{A}$ with the closed $G$-invariant subset

$$
\left\{x \in\left((\mathbb{R} / \mathbb{Z})^{G}\right)^{n}: x A^{*}=0_{\left((\mathbb{R} / \mathbb{Z})^{G}\right)^{n}}\right\}
$$

of $\left((\mathbb{R} / \mathbb{Z})^{G}\right)^{n}$ equipped with the action of $G$ by left translation. In the case that $A$ is invertible in $M_{n}\left(\ell^{1}(G)\right)$ the action $G \curvearrowright X_{A}$ is expansive, and in fact such actions and their restrictions to closed $G$-invariant subgroups constitute precisely all of the expansive algebraic actions [7, Theorem 3.1]. When $G$ is amenable, given an action of the form $G \curvearrowright X_{A}$ with $A$ invertible in $M_{n}\left(\ell^{1}(G)\right)$, every tuple of points in $X$ is an IE-tuple (see Lemma 5.4 and Theorems 7.3 and 7.8 in [7]). We will extend this result in two ways in Theorems 6.6 and 6.7, which demonstrate that in broader contexts independent behavior similarly saturates the structure of actions of the form $G \curvearrowright X_{A}$ with $A$ invertible in $M_{n}\left(\ell^{1}(G)\right)$.

First however we examine orbit IE-tuples in the context of actions $G \curvearrowright X$ on a compact metrizable (not necessarily Abelian) group by automorphisms. It was shown in [7, Theorem 7.3] that, when $G$ is amenable, the IE-tuples for such an action are determined by a closed $G$-invariant normal subgroup of $X$ called the IE group. We now proceed to record some observations showing that the basic theory of the IEgroup from [7] can be extended from amenable $G$ to general $G$ using orbit IE-tuples. Thus $G$ will be an arbitrary countable discrete group until we turn to the sofic setting in Theorem 6.7.

The proof of Lemma 3.11 in [21] shows the following:

Lemma 6.1 Let $G$ act continuously on a compact metrizable space $X$. Let $A$ be a Borel subset of $X$, and $\mu$ a $G$-invariant Borel probability measure on $X$. Then A has independence density at least $\mu(A)$ over $G$.

From Lemma 6.1 we immediately obtain:

Lemma 6.2 Let $G$ act continuously on a compact metrizable space $X$. Let $\mu$ be a $G$-invariant Borel probability measure on $X$. Then every point in the support of $\mu$ is an orbit IE-1-tuple.

We now suppose that $G$ acts continuously on a compact metrizable group $X$ by automorphisms. From Lemma 6.2 we have:

Lemma 6.3 Every point of $X$ is an orbit IE-1-tuple.

Denote by $\operatorname{IE}(X)$ the set of all $x \in X$ such that $\left(x, e_{X}\right)$ is an orbit IE-pair, where $e_{X}$ is the identity element of $X$. The proof of Theorem 7.3 in [7] shows the following.

Theorem 6.4 $\operatorname{IE}(X)$ is a closed $G$-invariant normal subgroup of $X$. For every $k \in \mathbb{N}$ the set $\operatorname{IE}_{k}(X, G)$ of all orbit IE-k-tuples is a closed $G$-invariant subgroup of the 
group $X^{k}$ and

$$
\begin{aligned}
\operatorname{IE}_{k}(X, G) & =\left\{\left(x_{1} y, \ldots, x_{k} y\right): x_{1}, \ldots, x_{k} \in \operatorname{IE}(X), y \in X\right\} \\
& =\left\{\left(y x_{1}, \ldots, y x_{k}\right): x_{1}, \ldots, x_{k} \in \operatorname{IE}(X), y \in X\right\} .
\end{aligned}
$$

Now we suppose that $X$ is Abelian. In this case a point $x \in X$ is said to be 1homoclinic if the function $s \mapsto \varphi(s x)-1$ on $G$ lies in $\ell^{1}(G)$ for every $\varphi$ in the Pontryagin dual $\widehat{X}$. The set of 1 -homoclinic points is written $\Delta^{1}(X)$. This set was studied in [29, 30, 37] in the case $G=\mathbb{Z}^{d}$ and in [7] for more general $G$. From the proof of Theorem 7.8 in [7] we obtain the following.

Theorem 6.5 Suppose that $\widehat{X}$ is a finitely generated left $\mathbb{Z} G$-module. Then $\Delta^{1}(X) \subseteq$ $\operatorname{IE}(X)$.

From Theorem 6.5 and [7, Lemma 5.4] we obtain:

Theorem 6.6 Let $n \in \mathbb{N}$, and let $A$ be an element of $M_{n}(\mathbb{Z} G)$ which is invertible in $M_{n}\left(\ell^{1}(G)\right)$. Then for the action $G \curvearrowright X_{A}$ one has $\operatorname{IE}\left(X_{A}\right)=X_{A}$.

Now we let $G$ be a countable sofic group and $\Sigma=\left\{\sigma_{i}: G \rightarrow \operatorname{Sym}\left(d_{i}\right)\right\}_{i=1}^{\infty}$ a sofic approximation sequence for $G$.

Theorem 6.7 Let $n \in \mathbb{N}$, and let $A$ be an element of $M_{n}(\mathbb{Z} G)$ which is invertible in $M_{n}\left(\ell^{1}(G)\right)$. Consider the action $G \curvearrowright X_{A}$. Then, for each $k \in \mathbb{N}$, every $k$-tuple of points in $X_{A}$ is a $\Sigma$-IE-tuple.

Before proceeding to the proof of Theorem 6.7, we give an application to a problem of Deninger. For an invertible element $f$ in the group von Neumann algebra $\mathcal{L} G$ of a countable discrete group $G$ the Fuglede-Kadison determinant is defined by $\operatorname{det}_{\mathcal{L} G} f=\exp \operatorname{tr}(\log |f|)$ where $\operatorname{tr}$ is the canonical trace on $\mathcal{L} G$. In [9, Question 26] Deninger asked whether $\operatorname{det}_{\mathcal{L} G} f>1$ whenever $f \in \mathbb{Z} G$ is invertible in $\ell^{1}(G)$ and has no left inverse in $\mathbb{Z} G$. An affirmative answer was given by Deninger and Schmidt in the case that $G$ is residually finite and amenable [10, Corollary 6.7] and more generally by Chung and the second author in the case $G$ is amenable [7, Corollary 7.9]. Using Theorem 6.7, Proposition 4.16(3), Theorem 7.1 in [23], and the argument in the proof of Corollary 7.9 in [7], we obtain an answer to Deninger's question for all countable residually finite groups:

Corollary 6.8 Suppose that $G$ is residually finite and that $f$ is an element of $\mathbb{Z} G$ which is invertible in $\ell^{1}(G)$ and has no left inverse in $\mathbb{Z} G$. Then $\operatorname{det}_{\mathcal{L} G} f>1$.

Let $n \in \mathbb{N}$. For $A=\left(A_{i j}\right)_{1 \leq i, j \leq n} \in M_{n}\left(\ell^{1}(G)\right)$, we set

$$
\|A\|_{1}=\sum_{1 \leq i, j \leq n}\left\|A_{i j}\right\|_{1} .
$$


For $\left(a_{1}, \ldots, a_{n}\right) \in \mathbb{R}^{d}$, we set $\left\|\left(a_{1}, \ldots, a_{n}\right)\right\|_{\infty}=\max _{1 \leq j \leq n}\left|a_{j}\right|$. For $\xi$ : $\{1, \ldots, d\} \rightarrow \mathbb{Z}^{n}$, we set

$$
\|\xi\|_{\infty}=\max _{1 \leq j \leq d}\|\xi(j)\|_{\infty} .
$$

Denote by $P$ the natural quotient map $\left(\mathbb{R}^{n}\right)^{G} \rightarrow\left((\mathbb{R} / \mathbb{Z})^{n}\right)^{G}$. Denote by $\rho$ the canonical metric on $\mathbb{R} / \mathbb{Z}$ defined by

$$
\rho\left(t_{1}+\mathbb{Z}, t_{2}+\mathbb{Z}\right):=\min _{m \in \mathbb{Z}}\left|t_{1}-t_{2}-m\right|
$$

By abuse of notation, we also use $\rho$ to denote the metric on $(\mathbb{R} / \mathbb{Z})^{n}$ defined by

$$
\rho\left(\left(a_{1}, \ldots, a_{n}\right),\left(b_{1}, \ldots, b_{n}\right)\right):=\max _{1 \leq j \leq n} \rho\left(a_{j}, b_{j}\right) .
$$

Via the coordinate map at the identity element of $G$, we will think of $\rho$ as a continuous pseudometric on $\left((\mathbb{R} / \mathbb{Z})^{n}\right)^{G}$.

Lemma 6.9 Let $n \in \mathbb{N}$, and let $A$ be an element of $M_{n}(\mathbb{Z} G)$ which is invertible in $M_{n}\left(\ell^{1}(G)\right)$. Consider the action $G \curvearrowright X_{A}$. Let $F$ be a nonempty finite subset of $G$ and let $M, \delta>0$. For every $d \in \mathbb{N}$, good enough sofic approximation $\sigma: G \rightarrow \operatorname{Sym}(d)$, and $\xi:\{1, \ldots, d\} \rightarrow \mathbb{Z}^{n}$ with $\|\xi\|_{\infty} \leq M$, if we define $h:\{1, \ldots, d\} \rightarrow\left(\mathbb{Z}^{n}\right)^{G}$ and $\varphi:\{1, \ldots, d\} \rightarrow X_{A}$ by

$$
(h(a))_{t^{-1}}=\xi(\text { ta }) \quad \text { for all } t \in G
$$

and

$$
\varphi(a)=P\left((h(a))\left(A^{*}\right)^{-1}\right)
$$

then $\varphi \in \operatorname{Map}(\rho, F, \delta, \sigma)$.

Proof Since $\left(A^{*}\right)^{-1} \in M_{d}\left(\ell^{1}(G)\right)$, there exists a nonempty finite subset $K$ of $G$ such that for all $z_{1}, z_{2} \in\left(\mathbb{Z}^{n}\right)^{G}$ such that $\left\|z_{1}\right\|_{\infty},\left\|z_{2}\right\|_{\infty} \leq M$ and $z_{1}, z_{2}$ coincide on $K$, one has $\left\|\left(z_{1}\left(A^{*}\right)^{-1}\right)_{e}-\left(z_{2}\left(A^{*}\right)^{-1}\right)_{e}\right\|_{\infty}<\delta / 2$, which implies that $\rho\left(P\left(z_{1}\left(A^{*}\right)^{-1}\right), P\left(z_{2}\left(A^{*}\right)^{-1}\right)\right)<\delta / 2$.

Denote by $\Lambda$ the set of all $a \in\{1, \ldots, d\}$ satisfying $t(s a)=(t s) a$ for all $t \in K^{-1}$ and $s \in F$. When $\sigma$ is a good enough sofic approximation for $G$, one has $|\Lambda| \geq$ $\left(1-(\delta / 2)^{2}\right) d$. Let $a \in \Lambda$ and $s \in F$. Then

$$
s(\varphi(a))=P\left((s(h(a)))\left(A^{*}\right)^{-1}\right)
$$

and

$$
\varphi(s a)=P\left((h(s a))\left(A^{*}\right)^{-1}\right) .
$$

For every $t \in K^{-1}$ one has

$$
(s(h(a)))_{t^{-1}}=(h(a))_{s^{-1} t^{-1}}=\xi((t s) a)=\xi(t(s a))=(h(s a))_{t^{-1}} .
$$


Thus, by the choice of $K$, we have $\rho(s(\varphi(a)), \varphi(s a))<\delta / 2$. Note that $\left((\mathbb{R} / \mathbb{Z})^{n}\right)^{G}$ has diameter 1 under $\rho$. It follows that

$$
\rho_{2}(s \varphi(\cdot), \varphi(s \cdot)) \leq\left((\delta / 2)^{2}+1-|\Lambda| / d\right)^{1 / 2}<\delta .
$$

Therefore $\varphi \in \operatorname{Map}(\rho, F, \delta, \sigma)$.

We are ready to prove Theorem 6.7.

Proof of Theorem 6.7 Let $\boldsymbol{x}=\left(x_{1}, \ldots, x_{k}\right)$ be a $k$-tuple of points in $X_{A}$. Then for each $1 \leq j \leq k$ there is a $z_{j} \in\left(\mathbb{Z}^{n}\right)^{G}$ such that $\left\|z_{j}\right\|_{\infty} \leq\|A\|_{1}$ and $x_{j}=$ $P\left(z_{j}\left(A^{*}\right)^{-1}\right)$.

Let $U_{1} \times \cdots \times U_{k}$ be a product neighborhood of $\boldsymbol{x}$ in $X^{k}$. Since the map from bounded subsets of $\left(\mathbb{Z}^{n}\right)^{G}$ equipped with the pointwise convergence topology to $X_{A}$ sending $z$ to $P\left(z\left(A^{*}\right)^{-1}\right)$ is continuous [10, Proposition 4.2], there is a nonempty finite subset $K$ of $G$ such that for every $1 \leq j \leq k$ and $z \in\left(\mathbb{Z}^{n}\right)^{G}$ with $\|z\|_{\infty} \leq\|A\|_{1}$ and $\left.z\right|_{K}=\left.z_{j}\right|_{K}$, one has $P\left(z\left(A^{*}\right)^{-1}\right) \in U_{j}$.

Let $F$ be a nonempty finite subset of $G$ and $\delta>0$. Let $d \in \mathbb{N}$ and let $\sigma$ be a map from $G$ to $\operatorname{Sym}(d)$. Denote by $\Lambda$ the set of all $a \in\{1, \ldots, d\}$ such that $s a \neq t a$ for all distinct $s, t \in K^{-1}$. When $\sigma$ is a good enough sofic approximation for $G$, we have $|\Lambda| \geq d / 2$. Let $\mathcal{J}$ be a maximal subset of $\Lambda$ subject to the condition that the sets $K^{-1} a$ for $a \in \mathcal{J}$ are pairwise disjoint. Then $\Lambda \subseteq\left(\sigma\left(K^{-1}\right)\right)^{-1} \sigma\left(K^{-1}\right) \mathcal{J}$, and hence $|\Lambda| \leq|K|^{2}|\mathcal{J}|$. Therefore $|\mathcal{J}| \geq d /\left(2|K|^{2}\right)$.

We claim that $\mathcal{J}$ is a $(\rho, F, \delta, \sigma)$-independence set for $\boldsymbol{U}=\left(U_{1}, \ldots, U_{k}\right)$ when $\sigma$ is a good enough sofic approximation for $G$. Let $\omega$ be a map from $\{1, \ldots, d\}$ to $\{1, \ldots, k\}$. Define $\xi:\{1, \ldots, d\} \rightarrow \mathbb{Z}^{n}$ by $\xi(t a)=\left(z_{\omega(a)}\right)_{t^{-1}}$ for all $a \in \mathcal{J}$ and $t \in$ $K^{-1}$, and $\xi(b)=0$ for all $b$ not in $K^{-1} \mathcal{J}$. Then we have $h:\{1, \ldots, d\} \rightarrow\left(\mathbb{Z}^{n}\right)^{G}$ and $\varphi \in \operatorname{Map}(\rho, F, \delta, \sigma)$ defined in Lemma 6.9 for $M=\|A\|_{1}$ when $\sigma$ is a good enough sofic approximation. Let $a \in \mathcal{J}$. For any $t \in K^{-1}$, one has

$$
(h(a))_{t^{-1}}=\xi(t a)=\left(z_{\omega(a)}\right)_{t^{-1}} .
$$

By the choice of $K$ we have $\varphi(a)=P\left(h(a)\left(A^{*}\right)^{-1}\right) \in U_{\omega(a)}$. This proves our claim, and finishes the proof of the theorem.

\section{Orbit IE-tuples and Untameness}

Let $G$ be a countably infinite group acting continuously on a compact Hausdorff space $X$.

Theorem 7.1 Let $k \in \mathbb{N}$ and let $\boldsymbol{A}$ be a $k$-tuple of subsets of $X$. Suppose that $\boldsymbol{A}$ has positive independence density over $G$. Then $A$ has an infinite independence set in $G$.

Proof Denote by $q$ the density of $\boldsymbol{A}$ over $G$.

Let $F_{1}$ be a nonempty finite subset of $G$. Take $s_{1}, s_{2}, \ldots$ in $G$ such that setting $F_{n+1}=F_{n} \cup F_{n} s_{n}$ for all $n \in \mathbb{N}$ one has $F_{n} \cap F_{n} s_{n}=\emptyset$ for all $n \in \mathbb{N}$. 
Let $n \in \mathbb{N}$. Take an independence set $E_{n}$ of $\boldsymbol{A}$ contained in $F_{n}$ with $\left|E_{n}\right| \geq q\left|F_{n}\right|$. We will construct, inductively on $m$, nonempty finite subsets $F_{m, k}^{(n)}$ and $E_{m}^{(n)}$ of $G$ for all $1 \leq m \leq k \leq n$ and $t_{m}^{(n)} \in G$ for all $1 \leq m<n$ such that

(1) $F_{n, n}^{(n)}=F_{n}$ and $E_{n}^{(n)}=E_{n}$;

(2) $t_{m}^{(n)}$ is equal to either $e$ or $s_{m}^{-1}$ for each $1 \leq m<n$;

(3) $F_{m, k}^{(n)}=F_{m+1, k}^{(n)} t_{m}^{(n)}$ and $F_{m, m}^{(n)}=F_{m}$ for all $1 \leq m<k \leq n$;

(4) $E_{m}^{(n)}=E_{m+1}^{(n)} t_{m}^{(n)}$ for each $1 \leq m<n$;

(5) $\left|E_{m}^{(n)} \cap F_{m, k}^{(n)}\right| \geq q\left|F_{m, k}^{(n)}\right|$ for all $1 \leq m \leq k \leq n$.

To start with, we define $F_{n, n}^{(n)}$ and $E_{n}^{(n)}$ according to (1). If $\left|E_{n}^{(n)} \cap F_{n-1}\right| \geq q\left|F_{n-1}\right|$, we set $t_{n-1}^{(n)}=e$. Otherwise, since $\left|E_{n}^{(n)} \cap F_{n, n}^{(n)}\right| \geq q\left|F_{n, n}^{(n)}\right|$ and $F_{n, n}^{(n)}=F_{n}$ is the disjoint union of $F_{n-1}$ and $F_{n-1} s_{n-1}$, we must have $\left|E_{n}^{(n)} \cap F_{n-1} s_{n-1}\right| \geq q\left|F_{n-1} s_{n-1}\right|$, and we set $t_{n-1}^{(n)}=s_{n-1}^{-1}$. Defining $F_{n-1, k}^{(n)}$ for $n-1 \leq k \leq n$ and $E_{n-1}^{(n)}$ according to (3) and (4) respectively, we have that (5) holds for $m=n-1$. Next, if $\left|E_{n-1}^{(n)} \cap F_{n-2}\right| \geq q\left|F_{n-2}\right|$, we set $t_{n-2}^{(n)}=e$. Otherwise, since $\left|E_{n-1}^{(n)} \cap F_{n-1, n-1}^{(n)}\right| \geq$ $q\left|F_{n-1, n-1}^{(n)}\right|$ and $F_{n-1, n-1}^{(n)}=F_{n-1}$ is the disjoint union of $F_{n-2}$ and $F_{n-2} s_{n-2}$, we must have $\left|E_{n-1}^{(n)} \cap F_{n-2} s_{n-2}\right| \geq q\left|F_{n-2} s_{n-2}\right|$, and we set $t_{n-2}^{(n)}=s_{n-2}^{-1}$. Defining $F_{n-2, k}^{(n)}$ for $n-2 \leq k \leq n$ and $E_{n-2}^{(n)}$ according to (3) and (4) respectively, we have that (5) holds for $m=n-2$. Continuing in this way, we define $F_{m, k}^{(n)}, E_{m}^{(n)}$, and $t_{m}^{(n)}$ satisfying the above conditions.

Note that if $E^{\prime}$ is an independence set for $\boldsymbol{A}$ in $G$, then $E^{\prime} s$ is an independence set for $\boldsymbol{A}$ in $G$ for all $s \in G$. By induction on $m$, we find easily that $E_{m}^{(n)}$ is an independence set for $\boldsymbol{A}$ in $G$ for all $n \in \mathbb{N}$ and $1 \leq m \leq n$. Also note that for any $1 \leq m \leq k \leq n, F_{m, k}^{(n)}$ depends only on $F_{k} \cap E_{k}^{(n)}$. In particular, for any fixed $k \in \mathbb{N}$ the number of sets appearing in $F_{1, k}^{(n)}$ for all $n \geq k$ is finite. Thus we can find a strictly increasing sequence $n_{1}<n_{2}<\cdots$ in $\mathbb{N}$ such that for any fixed $k \in \mathbb{N}$ the sets $F_{1, k}^{\left(n_{l}\right)}$ and $E_{1}^{\left(n_{l}\right)} \cap F_{1, k}^{\left(n_{l}\right)}$ do not depend on $l \geq k$. Set $E=\bigcup_{k \in \mathbb{N}}\left(E_{1}^{\left(n_{k}\right)} \cap F_{1, k}^{\left(n_{k}\right)}\right)$. Since $\left|E_{1}^{\left(n_{k}\right)} \cap F_{1, k}^{\left(n_{k}\right)}\right| \geq q\left|F_{1, k}^{\left(n_{k}\right)}\right|=q\left|F_{k}\right|=q\left|F_{1}\right| 2^{k-1}$ for every $k \in \mathbb{N}$, the set $E$ is infinite. For every $k \in \mathbb{N}$ one has

$$
\begin{aligned}
\left(E_{1}^{\left(n_{k+1}\right)} \cap F_{1, k+1}^{\left(n_{k+1}\right)}\right) \cap F_{1, k}^{\left(n_{k}\right)} & =\left(E_{1}^{\left(n_{k+1}\right)} \cap F_{1, k+1}^{\left(n_{k+1}\right)}\right) \cap F_{1, k}^{\left(n_{k+1}\right)} \\
& =E_{1}^{\left(n_{k+1}\right)} \cap F_{1, k}^{\left(n_{k+1}\right)} \\
& =E_{1}^{\left(n_{k}\right)} \cap F_{1, k}^{\left(n_{k}\right)} .
\end{aligned}
$$

Thus the sequence $\left\{E_{1}^{\left(n_{k}\right)} \cap F_{1, k}^{\left(n_{k}\right)}\right\}_{k \in \mathbb{N}}$ is increasing. Since the family of independence sets for $\boldsymbol{A}$ in $G$ is closed under taking increasing unions, we conclude that $E$ is an independence set for $\boldsymbol{A}$ in $G$.

Recall that a tuple $\left(x_{1}, \ldots, x_{k}\right) \in X^{k}$ is an IT-tuple if for every product neighborhood $U_{1} \times \cdots \times U_{k}$ of $\left(x_{1}, \ldots, x_{k}\right)$ the tuple $\left(U_{1}, \ldots, U_{k}\right)$ has an infinite independence set [21]. 
Corollary 7.2 Every orbit IE-tuple of the action $G \curvearrowright X$ is an IT-tuple.

Write $C(X)$ for the Banach space of continuous complex-valued functions on $X$ with the supremum norm. The action $G \curvearrowright X$ is said to be tame if no element $f \in$ $C(X)$ admits an infinite subset $J$ of $G$ such that, for $s$ ranging in $J$, the family of functions $x \mapsto f\left(s^{-1} x\right)$ in $C(X)$ is equivalent to the standard basis of $\ell^{1}$, meaning that there is a bijection between the two which extends to an isomorphism (i.e., a bounded linear map with bounded inverse) between the closures of their linear spans $[14,25]$. The action is tame if and only if there is no nondiagonal IT-pair in $X \times X$ [21, Proposition 6.4]. Thus from the above corollary we see that a tame action has no nondiagonal orbit IE-tuples.

\section{$8 \quad \Sigma$-IE-tuples and Li-Yorke Chaos}

Let $G$ be a countably infinite sofic group and $\Sigma=\left\{\sigma_{i}: G \rightarrow \operatorname{Sym}\left(d_{i}\right)\right\}_{i=1}^{\infty}$ a sofic approximation sequence for $G$. We fix a free ultrafilter $\mathfrak{F}$ on $\mathbb{N}$ and use it in the definitions of sofic topological entropy and $\Sigma$-IE-tuples, as in Sect. 5.

Let $G \curvearrowright X$ be a continuous action on a compact metrizable space. Let $\rho$ be a compatible metric on $X$. We say that $(x, y) \in X \times X$ is a Li-Yorke pair if

$$
\limsup _{G \ni s \rightarrow \infty} \rho(s x, s y)>0 \quad \text { and } \quad \liminf _{G \ni s \rightarrow \infty} \rho(s x, s y)=0,
$$

where the limit supremum and limit infimum mean the limits of $\sup _{s \in G \backslash F} \rho(s x, s y)$ and $\inf _{s \in G \backslash F} \rho(s x, s y)$, respectively, over the net of finite subsets $F$ of $G$. Note that the definition of Li-Yorke pair does not depend on the choice of the metric $\rho$. We say that the action $G \curvearrowright X$ is Li-Yorke chaotic if there is an uncountable subset $Z$ of $X$ such that every nondiagonal pair $(x, y)$ in $Z \times Z$ is a Li-Yorke pair. These definitions adapt those for continuous $\mathbb{N}$-actions, which have their origins in [28]. In that setting Blanchard, Glasner, Kolyada, and Maass showed that positive entropy implies Li-Yorke chaos [3]. The following theorem demonstrates that, in our sofic context, positive topological entropy with respect to some sofic approximation sequence implies Li-Yorke chaos [21, Theorem 3.18].

Theorem 8.1 Suppose that $k \geq 2$ and $\boldsymbol{x}=\left(x_{1}, \ldots, x_{k}\right)$ is a $\Sigma$-IE-tuple in $X^{k}$ with $x_{1}, \ldots, x_{k}$ pairwise distinct. For each $1 \leq j \leq k$, let $A_{j}$ be a neighborhood of $x_{j}$. Then there exist Cantor sets $Z_{j} \subseteq A_{j}$ for $j=1, \ldots, k$ such that the following hold:

(1) every nonempty finite tuple of points in $Z:=\bigcup_{j} Z_{j}$ is a $\Sigma$-IE-tuple;

(2) for all $m \in \mathbb{N}$, distinct $y_{1}, \ldots, y_{m} \in Z$, and $y_{1}^{\prime}, \ldots, y_{m}^{\prime} \in Z$ one has

$$
\liminf _{G \ni s \rightarrow \infty} \max _{1 \leq i \leq m} \rho\left(s y_{i}, y_{i}^{\prime}\right)=0 .
$$

We now set out to prove Theorem 8.1. We begin with the following lemmas.

Lemma 8.2 Let $k \geq 2$ and $\boldsymbol{A}=\left(A_{1}, \ldots, A_{k}\right)$ be a tuple of closed subsets of $X$ with positive upper independence density over $\Sigma$. For each $j=1, \ldots, k$ let $U_{j}$ be an open 
set containing $A_{j}$. Let $E$ be a finite subset of $G$. Then there exists an $s \in G \backslash E$ such that the tuple $\boldsymbol{A}^{\prime}$ consisting of $A_{i} \cap s^{-1} U_{j}$ for all $i, j=1, \ldots, k$ has positive upper independence density over $\Sigma$.

Proof Take $1<\lambda<\frac{k}{k-1}$. Then we have the constant $c>0$ in Lemma 4.5. Take a $q>0$ such that for every nonempty finite subset $F$ of $G$ and $\delta>0$ the set $V_{F, \delta}$ of all $i \in \mathbb{N}$ for which $\boldsymbol{A}$ has a $\left(\rho, F, \delta, \sigma_{i}\right)$-independence set of cardinality at least $q d_{i}$ is in $\mathfrak{F}$. Take a finite subset $W$ of $G$ such that $c q|W|>8$ and for any distinct $s, t \in$ $W$ one has $s^{-1} t \notin E$. When $0<|W|^{2} \kappa<1 / 2$, the number of subsets of $\{1, \ldots, d\}$ of cardinality no greater than $|W|^{2} \kappa d$ is equal to $\sum_{j=0}^{\left\lfloor|W|^{2} \kappa d\right\rfloor}\left(\begin{array}{c}d \\ j\end{array}\right)$, which is at most $|W|^{2} \kappa d\left(\begin{array}{c}d \\ |W|^{2} \kappa d\end{array}\right)$, which by Stirling's approximation is less than $\exp (\beta d)$ for some $\beta>0$ depending on $\kappa$ but not on $d$ when $d$ is sufficiently large with $\beta \rightarrow 0$ as $\kappa \rightarrow$ 0 . Take $c q /\left(2|W|^{2}\right)>\kappa>0$ such that for any $1 \leq j \leq k$ and $x \in X \backslash U_{j}$ one has $\rho\left(x, A_{j}\right)>\sqrt{\kappa}$ and for all sufficiently large $d \in \mathbb{N}$ the number of subsets of $\{1, \ldots, d\}$ of cardinality no greater than $|W|^{2} \kappa d$ is at most $\left(\frac{k}{(k-1) \lambda}\right)^{q d}$.

Let $F$ be a nonempty finite subset of $G$ and $\delta>0$. Set $F^{\prime}=F \cup W$ and $\delta^{\prime}=\min (\delta, \kappa)$. Let $i \in \mathbb{N}$ be such that $\boldsymbol{A}$ has a $\left(\rho, F^{\prime}, \delta^{\prime}, \sigma_{i}\right)$-independence set $\mathcal{J}_{i}$ of cardinality at least $q d_{i}$. For each $\omega \in\{1, \ldots, k\}^{\mathcal{J}_{i}}$ take a $\varphi_{\omega} \in \operatorname{Map}\left(\rho, F^{\prime}, \delta^{\prime}, \sigma_{i}\right)$ such that $\varphi_{\omega}(a) \in A_{\omega(a)}$ for every $a \in \mathcal{J}_{i}$. For each $\omega \in\{1, \ldots, k\} \mathcal{J}_{i}$, there is some $\Lambda_{\omega} \subseteq\left\{1, \ldots, d_{i}\right\}$ with $\left|\Lambda_{\omega}\right| \geq\left(1-|W|^{2} \delta^{\prime}\right) d_{i}$ such that $\rho\left(\varphi_{\omega}\left(\sigma_{i}(s) a\right), s \varphi_{\omega}(a)\right)<\sqrt{\delta^{\prime}}$ for all $s \in W^{-1} W$ and $a \in \Lambda_{\omega}$. By the choice of $\kappa$, when $i$ is large enough there is a subset $\Omega_{i}$ of $\{1, \ldots, k\}^{\mathcal{J}_{i}}$ with $\left(\frac{k}{(k-1) \lambda}\right)^{q d_{i}}\left|\Omega_{i}\right| \geq k^{\left|\mathcal{J}_{i}\right|}$ such that the set $\Lambda_{\omega}$ is the same, say $\Theta_{i}$, for every $\omega \in \Omega_{i}$, and $\left|\Theta_{i}\right| / d_{i} \geq 1-|W|^{2} \delta^{\prime}>1-c q / 2$. Then

$$
\left|\Omega_{i}\right| \geq k^{\left|\mathcal{J}_{i}\right|}\left(\frac{(k-1) \lambda}{k}\right)^{q d_{i}} \geq k^{\left|\mathcal{J}_{i}\right|}\left(\frac{(k-1) \lambda}{k}\right)^{\left|\mathcal{J}_{i}\right|}=((k-1) \lambda)^{\left|\mathcal{J}_{i}\right|}
$$

By our choice of $c$, we can find a subset $\mathcal{J}_{i}^{\prime}$ of $\mathcal{J}_{i}$ with $\left|\mathcal{J}_{i}^{\prime}\right| \geq c\left|\mathcal{J}_{i}\right| \geq c q d_{i}$ such that every map $\mathcal{J}_{i}^{\prime} \rightarrow\{1, \ldots, k\}$ extends to some $\omega \in \Omega_{i}$. When $i$ is large enough, one also has $\left|\mathcal{W}_{i}\right| \geq(1-c q / 4) d_{i}$ for the set

$$
\begin{gathered}
\mathcal{W}_{i}=\left\{a \in\left\{1, \ldots, d_{i}\right\}:\left(\left(\sigma_{i}(s)\right)^{-1} \sigma_{i}(t)\right)(a)=\sigma_{i}\left(s^{-1} t\right)(a) \text { for all } s, t \in W\right. \\
\text { and } \left.\sigma_{i}(s)(a) \neq a \text { for all } s \in W^{-1} W \backslash\{e\}\right\} .
\end{gathered}
$$

Note that $\left|\mathcal{W}_{i} \cap \Theta_{i} \cap \mathcal{J}_{i}^{\prime}\right| \geq c q d_{i} / 4$ and every map $\mathcal{W}_{i} \cap \Theta_{i} \cap \mathcal{J}_{i}^{\prime} \rightarrow\{1, \ldots, k\}$ extends to some $\omega \in \Omega_{i}$.

Denote by $\eta$ the maximum of $\left|\sigma_{i}(s)\left(\mathcal{W}_{i} \cap \Theta_{i} \cap \mathcal{J}_{i}^{\prime}\right) \cap \sigma_{i}(t)\left(\mathcal{W}_{i} \cap \Theta_{i} \cap \mathcal{J}_{i}^{\prime}\right)\right| / d_{i}$ for $s, t$ ranging over distinct elements of $W$. Then for each $s \in W$ there is a subset $\Upsilon_{i, s}$ of $\sigma_{i}(s)\left(\mathcal{W}_{i} \cap \Theta_{i} \cap \mathcal{J}_{i}^{\prime}\right)$ with cardinality at most $\eta|W| d_{i}$ such that the sets $\left(\sigma_{i}(s)\left(\mathcal{W}_{i} \cap\right.\right.$ $\left.\left.\Theta_{i} \cap \mathcal{J}_{i}^{\prime}\right)\right) \backslash \Upsilon_{i, s}$ for $s \in W$ are pairwise disjoint. It follows that 


$$
\begin{aligned}
\sum_{s \in W}\left|\sigma_{i}(s)\left(\mathcal{W}_{i} \cap \Theta_{i} \cap \mathcal{J}_{i}^{\prime}\right)\right| & \leq \eta|W|^{2} d_{i}+\left|\bigcup_{s \in W}\left(\left(\sigma_{i}(s)\left(\mathcal{W}_{i} \cap \Theta_{i} \cap \mathcal{J}_{i}^{\prime}\right)\right) \backslash \Upsilon_{i, s}\right)\right| \\
& \leq \eta|W|^{2} d_{i}+d_{i} .
\end{aligned}
$$

On the other hand, we have

$$
\sum_{s \in W}\left|\sigma_{i}(s)\left(\mathcal{W}_{i} \cap \Theta_{i} \cap \mathcal{J}_{i}^{\prime}\right)\right|=|W| \cdot\left|\mathcal{W}_{i} \cap \Theta_{i} \cap \mathcal{J}_{i}^{\prime}\right| \geq|W| c q d_{i} / 4 \geq 2 d_{i}
$$

Thus $\eta \geq 1 /|W|^{2}$. Then we can find some distinct $t_{i}, t_{i}^{\prime} \in W$ with $\mid \sigma_{i}\left(t_{i}\right)\left(\mathcal{W}_{i} \cap \Theta_{i} \cap\right.$ $\left.\mathcal{J}_{i}^{\prime}\right)\left.\cap \sigma_{i}\left(t_{i}^{\prime}\right)\left(\mathcal{W}_{i} \cap \Theta_{i} \cap \mathcal{J}_{i}^{\prime}\right)\left|\geq d_{i} /\right| W\right|^{2}$. Set $s_{i}=t_{i}^{-1} t_{i}^{\prime}$. Then $s_{i} \in W^{-1} W \backslash\{e\}$, and

$$
\begin{aligned}
& \left|\left(\mathcal{W}_{i} \cap \Theta_{i} \cap \mathcal{J}_{i}^{\prime}\right) \cap\left(\sigma_{i}\left(s_{i}\right)\right)^{-1}\left(\mathcal{W}_{i} \cap \Theta_{i} \cap \mathcal{J}_{i}^{\prime}\right)\right| \\
& \quad=\left|\left(\mathcal{W}_{i} \cap \Theta_{i} \cap \mathcal{J}_{i}^{\prime}\right) \cap \sigma_{i}\left(s_{i}\right)\left(\mathcal{W}_{i} \cap \Theta_{i} \cap \mathcal{J}_{i}^{\prime}\right)\right| \\
& \quad=\left|\sigma_{i}\left(t_{i}\right)\left(\mathcal{W}_{i} \cap \Theta_{i} \cap \mathcal{J}_{i}^{\prime}\right) \cap \sigma_{i}\left(t_{i}^{\prime}\right)\left(\mathcal{W}_{i} \cap \Theta_{i} \cap \mathcal{J}_{i}^{\prime}\right)\right| \\
& \quad \geq d_{i} /|W|^{2} .
\end{aligned}
$$

Take a maximal subset $\Xi_{i}$ of $\left(\mathcal{W}_{i} \cap \Theta_{i} \cap \mathcal{J}_{i}^{\prime}\right) \cap\left(\sigma_{i}\left(s_{i}\right)\right)^{-1}\left(\mathcal{W}_{i} \cap \Theta_{i} \cap \mathcal{J}_{i}^{\prime}\right)$ subject to the condition that for any $a \in \Xi_{i}$, neither $\sigma_{i}\left(s_{i}\right)(a)$ nor $\left(\sigma_{i}\left(s_{i}\right)\right)^{-1}(a)$ is in $\Xi_{i}$. Then $\Xi_{i} \cup \sigma_{i}\left(s_{i}\right) \Xi_{i} \cup\left(\sigma_{i}\left(s_{i}\right)\right)^{-1} \Xi_{i} \supseteq\left(\mathcal{W}_{i} \cap \Theta_{i} \cap \mathcal{J}_{i}^{\prime}\right) \cap\left(\sigma_{i}\left(s_{i}\right)\right)^{-1}\left(\mathcal{W}_{i} \cap \Theta_{i} \cap \mathcal{J}_{i}^{\prime}\right)$. It follows that $\left|\Xi_{i}\right| \geq\left|\left(\mathcal{W}_{i} \cap \Theta_{i} \cap \mathcal{J}_{i}^{\prime}\right) \cap\left(\sigma_{i}\left(s_{i}\right)\right)^{-1}\left(\mathcal{W}_{i} \cap \Theta_{i} \cap \mathcal{J}_{i}^{\prime}\right)\right| / 3 \geq d_{i} /\left(3|W|^{2}\right)$. Note that $\Xi_{i}$ and $\sigma_{i}\left(s_{i}\right) \Xi_{i}$ are disjoint subsets of $\mathcal{W}_{i} \cap \Theta_{i} \cap \mathcal{J}_{i}^{\prime}$.

Let $\xi=\left(\xi_{1}, \xi_{2}\right): \Xi_{i} \rightarrow\{1, \ldots, k\}^{2}$. Define a map $\xi^{\prime}: \Xi_{i} \cup \sigma_{i}\left(s_{i}\right) \Xi_{i} \rightarrow\{1, \ldots, k\}$ by $\xi^{\prime}(a)=\xi_{1}(a)$ and $\xi^{\prime}\left(\sigma_{i}\left(s_{i}\right)(a)\right)=\xi_{2}(a)$ for all $a \in \Xi_{i}$. Extend $\xi^{\prime}$ to some $\omega \in \Omega_{i}$. Then $\varphi_{\omega} \in \operatorname{Map}\left(\rho, F, \delta, \sigma_{i}\right), \varphi_{\omega}(a) \in A_{\omega(a)}=A_{\xi_{1}(a)}$ and $\varphi_{\omega}\left(\sigma_{i}\left(s_{i}\right)(a)\right) \in$ $A_{\omega\left(\sigma_{i}\left(s_{i}\right)(a)\right)}=A_{\xi_{2}(a)}$ for all $a \in \Xi_{i}$. For any $a \in \Xi_{i}$, since $\rho\left(\varphi_{\omega}\left(\sigma_{i}\left(s_{i}\right) a\right), s_{i} \varphi_{\omega}(a)\right)<$ $\sqrt{\delta^{\prime}} \leq \sqrt{\kappa}$, by the choice of $\kappa$ we have $s_{i} \varphi_{\omega}(a) \in U_{\xi_{2}}(a)$, and hence $\varphi_{\omega}(a) \in$ $A_{\xi_{1}(a)} \cap s_{i}^{-1} U_{\xi_{2}(a)}$. Therefore $\Xi_{i}$ is a $\left(\rho, F, \delta, \sigma_{i}\right)$-independence set of cardinality at least $d_{i} /\left(3|W|^{2}\right)$ for the tuple consisting of $A_{l} \cap s_{i}^{-1} U_{j}$ for all $l, j=1, \ldots, k$.

There is some $s_{F, \delta} \in W^{-1} W \backslash\{e\}$ such that the set of $i \in V_{F^{\prime}, \delta^{\prime}}$ for which $s_{i}$ is defined and $s_{i}=s_{F, \delta}$ lies in $\mathfrak{F}$. It follows that we can find an $s \in W^{-1} W \backslash\{e\}$ such that for any nonempty finite subset $F$ of $G$ and $\delta>0$ there are some nonempty finite subset $\tilde{F}$ of $G$ and $\tilde{\delta}>0$ with $F \subseteq \tilde{F}$ and $\delta>\tilde{\delta}$ such that $s_{\tilde{F}, \tilde{\delta}}=s$. Then the tuple $A^{\prime}$ consisting of $A_{l} \cap s^{-1} U_{j}$ for all $l, j=1, \ldots, k$ has upper independence density at least $1 /\left(3|W|^{2}\right)$ over $\Sigma$. From the choice of $W$ we have $s \notin E$.

From Lemma 8.2 by induction on $m$ we have:

Lemma 8.3 Let $k \geq 2$ and $\boldsymbol{A}=\left(A_{1}, \ldots, A_{k}\right)$ be a tuple of closed subsets of $X$ with positive upper independence density over $\Sigma$. For each $j=1, \ldots, k$ let $U_{j}$ be an open set containing $A_{j}$. Let $E$ be a finite subset of $G$ and $m \in \mathbb{N}$. Then there exist $s_{1}, \ldots, s_{m} \in G \backslash E$ such that $s_{i}^{-1} s_{j} \notin E$ for all distinct $1 \leq i, j \leq m$ and the tuple $A^{\prime}$ 
consisting of $A_{i} \cap s_{1}^{-1} U_{\omega(1)} \cap \cdots \cap s_{m}^{-1} U_{\omega(m)}$ for all $1 \leq i \leq k$ and $\omega \in\{1, \ldots, k\}^{m}$ has positive upper independence density over $\Sigma$.

We are ready to prove Theorem 8.1 .

Proof of Theorem 8.1 We may assume that the $A_{j}$ are closed and pairwise disjoint. Take an increasing sequence $E_{1} \subseteq E_{2} \subseteq \cdots$ of finite subsets of $G$ with union $G$. We shall construct, via induction on $m$, closed nonempty subsets $A_{m, j}$ of $X$ for $1 \leq j \leq$ $k^{2^{m-1}}$ with the following properties:

(a) $A_{1, j}=A_{j}$ for all $1 \leq j \leq k$,

(b) for every $m \geq 2$ and $1 \leq i \leq k^{2^{m-2}}, A_{m-1, i}$ contains exactly $k^{2^{m-2}}$ of the $A_{m, j}$ for $1 \leq j \leq k^{2^{m-1}}$

(c) for every $m \geq 2$ and map $\gamma:\left\{1, \ldots, k^{2^{m-1}}\right\} \rightarrow\left\{1, \ldots, k^{2^{m-2}}\right\}$ there exists a $t_{\gamma} \in$ $G \backslash E_{m-1}$ such that $t_{\gamma} A_{m, j} \subseteq \overline{U_{m-1, \gamma(j)}}$ for all $1 \leq j \leq k^{2^{m-1}}$, where $U_{m-1, i}=$ $\left\{x \in X: \rho\left(x, A_{m-1, i}\right)<2^{-m} \delta_{m-1}\right\}$ for all $1 \leq i \leq k^{2^{m-2}}$ and $\delta_{m-1}=\min \rho(x, y)$ for $x, y$ ranging over points in distinct $A_{m-1, j}$,

(d) when $m \geq 2, \operatorname{diam}\left(A_{m, j}\right) \leq 2^{-m}$ for all $1 \leq j \leq k^{2^{m-1}}$,

(e) for every $m$, the sets $A_{m, j}$ for $1 \leq j \leq k^{2^{m-1}}$ are pairwise disjoint,

(f) for every $m$, the collection $\left\{A_{m, j}: 1 \leq j \leq k^{2^{m-1}}\right\}$, ordered into a tuple, has positive upper independence density over $\Sigma$.

Suppose that we have constructed such $A_{m, j}$ over all $m$. Properties (b), (d) and (e) imply that $Z=\bigcap_{m \in \mathbb{N}} \bigcup_{j=1}^{k^{2^{m-1}}} A_{m, j}$ is a Cantor set. Property (a) implies that $Z_{j}:=Z \cap A_{j}$ is also a Cantor set for each $1 \leq j \leq k$. Condition (1) follows from properties (d) and (f). Condition (2) follows from properties (c) and (d).

We now construct the $A_{m, j}$. Define $A_{1, j}$ for $1 \leq j \leq k$ according to property (a). By assumption properties (e) and (f) are satisfied for $m=1$. Assume that we have constructed $A_{m}, j$ for all $j=1, \ldots, k^{2^{m-1}}$ with the above properties. Set $n=1+\left(k^{2^{m-1}}\right)^{k^{2^{m}}}$. By Lemma 8.3 we can find $s_{1}, \ldots, s_{n} \in G \backslash E_{m}$ such that the tuple consisting of $A_{m, i} \cap s_{1}^{-1} U_{m, \omega(1)} \cap \cdots \cap s_{n}^{-1} U_{m, \omega(n)}$ for all $1 \leq i \leq k^{2^{m-1}}$ and $\omega \in\left\{1, \ldots, k^{2^{m-1}}\right\}^{n}$ has positive upper independence density over $\Sigma$. Take a bijection $\varphi:\left\{1, \ldots, k^{2^{m-1}}\right\}^{\left\{1, \ldots, k^{2^{m}}\right\}} \rightarrow\{2, \ldots, n\}$. For each $\gamma:\left\{1, \ldots, k^{2^{m}}\right\} \rightarrow\left\{1, \ldots, k^{2^{m-1}}\right\}$, set $t_{\gamma}=s_{\varphi(\gamma)}$. For all $1 \leq i, j \leq k^{2^{m-1}}$, define $\omega_{i, j} \in\left\{1, \ldots, k^{2^{m-1}}\right\}^{n}$ by $\omega_{i, j}(1)=j$ and $\omega_{i, j}(\varphi(\gamma))=\gamma\left((i-1) k^{2^{m-1}}+j\right)$ for all $\gamma \in\left\{1, \ldots, k^{2^{m-1}}\right\}^{\left\{1, \ldots, k^{2^{m}}\right\}}$, and set $A_{m+1,(i-1) k^{2 m-1}+j}=A_{m, i} \cap s_{1}^{-1} \overline{U_{m, \omega_{i, j}(1)}} \cap \cdots \cap s_{n}^{-1} \overline{U_{m, \omega_{i, j}(n)}}$. Then properties (b), (c), (e) and (f) hold for $m+1$. For each $1 \leq j \leq k^{2^{m}}$ write $A_{m+1, j}$ as the union of finitely many closed subsets each with diameter no bigger than $2^{-(m+1)}$. Using Lemma 4.13 we may replace $A_{m+1, j}$ by one of these subsets. Consequently, property (d) is also satisfied for $m+1$. This completes the induction procedure and hence the proof of the theorem.

Corollary 8.4 If $h_{\Sigma}(X, G)>0$ for some sofic approximation sequence $\Sigma$ then the action is Li-Yorke chaotic. 
An action $G \curvearrowright X$ is said to be distal if $\inf _{s \in G} \rho(s x, s y)>0$ for all distinct $x, y \in X$. We refer the reader to [2] for the basics of distal actions. Since distal actions have no Li-Yorke pairs, from Corollary 8.4 we obtain the following consequence, which extends the result of Parry that distal integer actions on compact metrizable spaces have zero entropy [34]. For amenable $G$ we write $h_{\text {top }}(X, G)$ for the classical topological entropy, which is equal to the sofic entropy $h_{\Sigma}(X, G)$ for every $\Sigma$ [24].

Corollary 8.5 If the action $G \curvearrowright X$ is distal, then $h_{\Sigma}(X, G)=0$ or $-\infty$. In particular, if $G$ is amenable and $G \curvearrowright X$ is distal, then $h_{\mathrm{top}}(X, G)=0$.

We remark that every distal action has an invariant Borel probability measure [2, p. 125], [39, p. 496]. But we do not know whether one can conclude that $h_{\Sigma}(X, G)=$ 0 in Corollary 8.5 .

Acknowledgements The first author was partially supported by NSF grants DMS-0900938 and DMS1162309. The second author was partially supported by NSF grant DMS-1001625. We are grateful to Wen Huang, Liviu Paunescu and Xiangdong Ye for helpful comments.

\section{References}

1. Alekseev, M.A., Glebskiǐ, L.Yu., Gordon, E.I.: On approximations of groups, group actions and Hopf algebras. In: Zap. Nauchn. Sem. S.-Peterburg. Otdel. Mat. Inst. Steklov. (POMI), vol. 256, POMI, St. Petersburg (1999). Teor. Predst. Din. Sist. Komb. i Algoritm. Metody. 3, 224-262, 268 [translation in J. Math. Sci. (New York) 107(5), 4305-4332 (2001)]

2. Auslander, J.: Minimal Flows and Their Extensions. North-Holland Mathematics Studies, vol. 153. North-Holland, Amsterdam (1988)

3. Blanchard, F., Glasner, E., Kolyada, S., Maass, A.: On Li-Yorke pairs. J. Reine Angew. Math. 547, 51-68 (2002)

4. Blanchard, F., Lacroix, Y.: Zero entropy factors of topological flows. Proc. Am. Math. Soc. 119(3), 985-992 (1993)

5. Bowen, L.: Measure conjugacy invariants for actions of countable sofic groups. J. Am. Math. Soc. 23(1), 217-245 (2010)

6. Bowen, L.: Entropy for expansive algebraic actions of residually finite groups. Ergod. Theory Dyn. Syst. 31(3), 703-718 (2011)

7. Chung, N.-P., Li, H.: Homoclinic groups, IE groups, and expansive algebraic actions. Preprint, 2011

8. Deninger, C.: Fuglede-Kadison determinants and entropy for actions of discrete amenable groups. J. Am. Math. Soc. 19(3), 737-758 (2006)

9. Deninger, C.: Mahler measures and Fuglede-Kadison determinants. Münster J. Math. 2, 45-63 (2009)

10. Deninger, C., Schmidt, K.: Expansive algebraic actions of discrete residually finite amenable groups and their entropy. Ergod. Theory Dyn. Syst. 27(3), 769-786 (2007)

11. Einsiedler, M., Ward, T.: Ergodic Theory, with a View Towards Number Theory. Springer, London (2011)

12. Elek, G., Szabo, E.: Sofic representations of amenable groups. Proc. Am. Math. Soc. 139, 4285-4291 (2011)

13. Glasner, E.: Ergodic Theory Via Joinings. Am. Mat. Soc., Providence (2003)

14. Glasner, E.: On tame enveloping semigroups. Colloq. Math. 105(2), 283-295 (2006)

15. Glasner, E., Weiss, B.: Quasi-factors of zero entropy systems. J. Am. Math. Soc. 8(3), 665-686 (1995)

16. Glasner, E., Ye, X.: Local entropy theory. Ergod. Theory Dyn. Syst. 29(2), 321-356 (2009)

17. Gromov, M.: Endomorphisms of symbolic algebraic varieties. J. Eur. Math. Soc. 1(2), 109-197 (1999)

18. Huang, W., Ye, X.: A local variational relation and applications. Isr. J. Math. 151, 237-279 (2006) 
19. Karpovsky, M.G., Milman, V.D.: Coordinate density of sets of vectors. Discrete Math. 24(2), 177-184 (1978)

20. Kerr, D.: Sofic measure entropy via finite partitions. Groups Geom. Dyn., in press

21. Kerr, D., Li, H.: Independence in topological and $C^{*}$-dynamics. Math. Ann. 338(4), 869-926 (2007)

22. Kerr, D., Li, H.: Combinatorial independence in measurable dynamics. J. Funct. Anal. 256(5), 13411386 (2009)

23. Kerr, D., Li, H.: Entropy and the variational principle for actions of sofic groups. Invent. Math. 186(3), 501-558 (2011)

24. Kerr, D., Li, H.: Soficity, amenability, and dynamical entropy. Am. J. Math., in press

25. Köhler, A.: Enveloping semigroups for flows. Proc. R. Ir. Acad., Sect. A 95(2), 179-191 (1995)

26. Li, H.: Compact group automorphisms, addition formulas and Fuglede-Kadison determinants. Ann. Math. (2) 176(1), 303-347 (2012)

27. Li, H., Thom, A.: Entropy, determinants, and $L^{2}$-torsion. Preprint, 2012

28. Li, T.Y., Yorke, J.A.: Period three implies chaos. Am. Math. Mon. 82(10), 985-992 (1975)

29. Lind, D., Schmidt, K., Verbitskiy, E.: Entropy and growth rate of periodic points of algebraic $\mathbb{Z}^{d}$ actions. In: Dynamical Numbers: Interplay Between Dynamical Systems and Number Theory. Contemp. Math., vol. 532, pp. 195-211. Am. Math. Soc., Providence (2010)

30. Lind, D., Schmidt, K., Verbitskiy, E.: Homoclinic points, atoral polynomials, and periodic points of algebraic $\mathbb{Z}^{d}$-actions. Ergod. Theory Dyn. Syst. doi:10.1017/S014338571200017X

31. Lind, D., Schmidt, K., Ward, T.: Mahler measure and entropy for commuting automorphisms of compact groups. Invent. Math. 101(3), 593-629 (1990)

32. Loeb, P.A.: Conversion from nonstandard to standard measure spaces and applications in probability theory. Trans. Am. Math. Soc. 211, 113-122 (1975)

33. Ornstein, D.S., Weiss, B.: Entropy and isomorphism theorems for actions of amenable groups. J. Anal. Math. 48, 1-141 (1987)

34. Parry, W.: Zero entropy of distal and related transformations. In: Topological Dynamics, Symposium, Colorado State Univ., Ft. Collins, CO, 1967, pp. 383-389. Benjamin, New York (1968)

35. Paunescu, L.: A convex structure on sofic embeddings. Ergod. Theory Dynam. Syst., in press

36. Sauer, N.: On the density of families of sets. J. Comb. Theory, Ser. A 13, 145-147 (1972)

37. Schmidt, K., Verbitskiy, E.: Abelian sandpiles and the harmonic model. Commun. Math. Phys. 292(3), 721-759 (2009)

38. Shelah, S.: A combinatorial problem; stability and order for models and theories in infinitary languages. Pac. J. Math. 41, 247-261 (1972)

39. de Vries, J.: Elements of Topological Dynamics. Mathematics and Its Applications, vol. 257. Kluwer Academic, Dordrecht (1993)

40. Zhang, G.: Local variational principle concerning entropy of sofic group action. J. Funct. Anal. 262, 1954-1985 (2012) 